\title{
Scattering Radiation Pattern Atlas: What anisotropic elastic properties can body waves resolve?
}

Vladimir Kazei ${ }^{1} \&$ Tariq Alkhalifah ${ }^{1}$

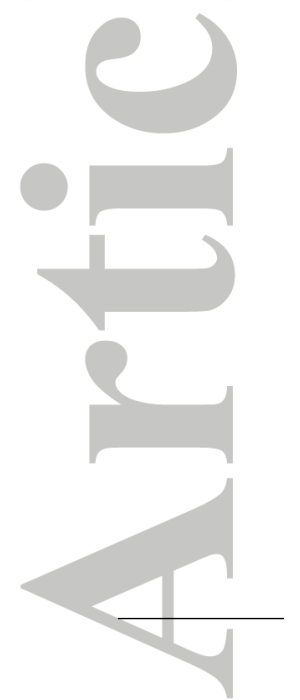

Vladimir Kazei, vladimir.kazei@kaust.edu.sa

${ }^{1}$ King Abdullah University of Science and

Technology (KAUST), Thuwal,

Saudi-Arabia

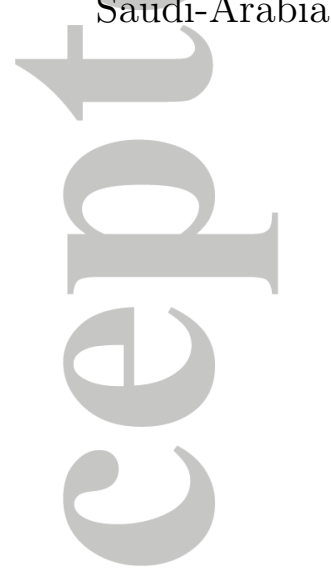

This article has been accepted for publication and undergone full peer review but has not been through the copyediting, typesetting, pagination and proofreading process, which may lead to differences between this version and the Version of Record. Please cite this article as doi: 10.1029/2018JB016687

(C)2019 American Geophysical Union. All Rights Reserved. 
Abstract. Full-waveform inversion (FWI) optimizes the subsurface properties of geophysical earth models in such a way that the modeled data, based on these subsurface properties, match the observed data. The anisotropic properties, whether monoclinic, orthorhombic, triclinic, or vertical transversally isotropic (VTI), of the subsurface, be it a fractured reservoir or the core-mantle boundary, are necessary to describe the observed wave phenomena. There are no principal limitations on the complexity of the anisotropy that can be inverted using FWI. However, the question remains - what kind of anisotropic descriptions of the elastic properties of the earth can or cannot be inverted reliably from seismic waveforms? We reveal the resolution that can be achieved through reconstructions of each elastic parameter by building vertical resolution patterns from the scattering radiation patterns of body waves. A visual analysis of these patterns indicates "tradeoffs", i.e., perturbations of parameters that have the same reflection-based scattering patterns as other perturbations. Each tradeoff leads to an apparent ambiguity in the inversion, which must be addressed by additional assumptions, constraints, or regularizations. For orthorhombic media, we find the exact tradeoffs that exist between parameters. Our parameterization isolates the VTI parameters and, therefore, we also obtain tradeoffs for every scattering mode for VTI media. We discover that only monoclinic parameters are recoverable from the first order scattering of monotypic waves. We summarize the tradeoffs found in tables for easy reference. This paper is intended to be useful for researchers

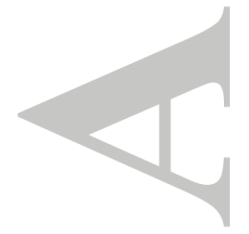

(C)2019 American Geophysical Union. All Rights Reserved. 
setting up anisotropic FWI problems, interpreting or controlling the quality of such inversions.

\section{Keypoints:}

- We share the resolution limits for each anisotropic parameter in waveform inversion

- For orthorhombic and VTI media we map the null-space of the inver-

sion

- Converted waves are necessary to move the inversion beyond monoclinic anisotropy type
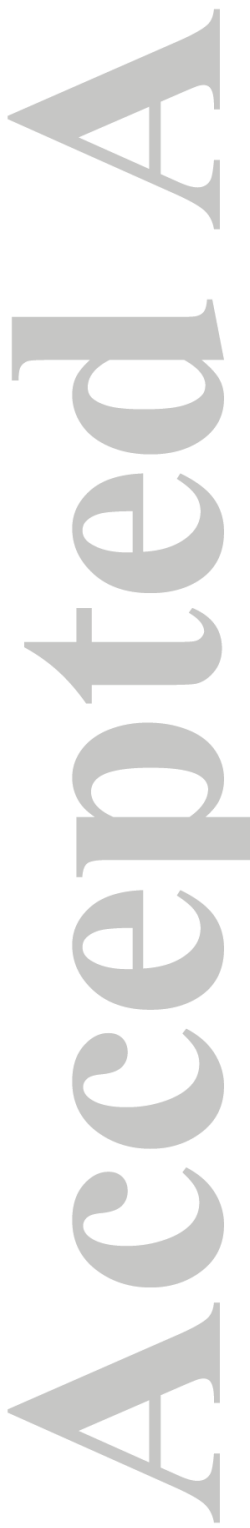

(C)2019 American Geophysical Union. All Rights Reserved. 


\section{Introduction}

A realistic representation of propagating wavefields is key to creating accurate subsurface models in global and exploration seismology. Elastic anisotropy, which can be classified and decomposed based upon the symmetries present in the anisotropic media (Browaeys \& Chevrot, 2004 ; Bóna, Bucataru, \& Slawinski, 2007), strongly influences the propagation of wavefields throughout the earth's interior. In global seismology, inversions of the anisotropy of the inner core (Tromp, 1993 ; Creager, 1999) and some regions of the earth's mantle (Long \& Becker, 2010 ; Bozdăg et al., 2016) have been used to construct more realistic global models for a better understanding of the physics of the earth. However, it is difficult to predict the optimal constraints, regularizations, and parameterizations to apply when designing elastic anisotropic inversions.

Tradeoffs are another major challenge encountered when inverting the anisotropic elastic properties of the earth's subsurface. Tradeoffs, also called couplings, between anisotropic parameters occur when different combinations of parameter perturbations lead to almost identical scattering patterns, i.e., the different perturbations produce the same elastic wave recordings. The problem of parameter tradeoffs is not new and has been extensively studied in isotropic media (R.-S. Wu \& Aki, 1985 ; Tarantola, 1986 ; Virieux \& Operto, 2009 ; Köhn, De Nil, Kurzmann, Przebindowska, \& Bohlen, 2012 ; Anikiev et al., 2014 ; Stenhjem Hagen \& Arntsen, 2017), vertical transversally isotropic (VTI) media (Eaton \& Stewart, 1994 ; Gholami, Brossier, Operto, Ribodetti, \& Virieux, 2013a ; Alkhalifah \& Plessix, 2014 ; He \& Plessix, 2016), and orthorhombic media (De Hoop, Spencer, \& Burridge, 1999 ; R. K. Shaw \& Sen, 2004 ; J.-W. Oh \& Alkhalifah, 2016 ; Kazei

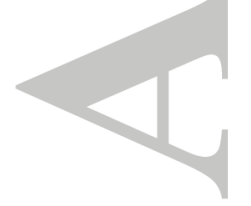

(C)2019 American Geophysical Union. All Rights Reserved. 
\& Alkhalifah, 2017). (Köhn, Hellwig, De Nil, \& Rabbel, 2015) performed a synthetic feasibility investigation on FWI for all triclinic elastic parameters, excluding density from the inversion. In this paper, we address the tradeoffs that inevitably arise in general elastic anisotropic inversions by explicitly defining those combinations that lead to similar recordings.

We consider the four most common cases of seismic anisotropy: VTI, orthorhombic (orthotropic), monoclinic, and triclinic anisotropy. VTI anisotropy is naturally induced in the subsurface by gravity-related vertical stress (Thomsen, 1986) and thin layering. Transversally isotropic media have one axis of rotational symmetry, which is often vertical. Therefore, the body waves propagate with the same velocity in any horizontal direction, but have different velocities depending on the deviation of the propagation direction from vertical (angle $\theta$ in Fig. 1(a)). Orthorhombic anisotropy characterizes most fractured carbonate reservoirs (Schoenberg \& Helbig, 1997 ; Tsvankin, 1997) and, therefore, plays a dominant role in exploration seismology. Orthorhombic anisotropy is also found in olivine crystals (Durham \& Goetze, 1977), which comprise a significant part of the earth's mantle and deeper crust (Tommasi et al., 2009). An orthorhombic medium can be viewed as a combination of two transversally isotropic media, one with a vertical axis of symmetry (layering) and another with a horizontal axis of symmetry (e.g., fractures). Wave propagation in such a medium depends on the azimuth (angle $\varphi$ in Fig. 1(a)). Even the kinematics of wave propagation in orthorhombic media are rather complicated and the subject of much current research (Stovas, 2015, 2017 ; Ivanov \& Stovas, 2016 ; Xu, Stovas, \& Sripanich, 2018). Monoclinic anisotropy is sometimes found in more complex geological formations or multiple sets of fractures (Grechka, Contreras, \& Tsvankin, 2000). A

(C)2019 American Geophysical Union. All Rights Reserved. 
triclinic anisotropic medium, the most common in the earth, has no planes of symmetry. Any elastic solid can be described by twenty-one elastic constants and density - triclinic anisotropic media require all twenty-two of these parameters for a complete description. Unfortunately, independent inversion for all twenty-two parameters is still a big challenge (Köhn et al., 2015) due to various tradeoffs. We try to understand which parameters are principally recoverable from different types of scattering and at which resolution.

The paper is organized as follows. In Section 2, we provide the history and general properties of scattering functions and the diffraction, reflection, and transmission of scattering radiation patterns. In Section 3, we introduce spectral sensitivities in order to directly access the vertical resolution lengths in inversion. In Section 4, we provide an atlas of these sensitivities for all possible parameter perturbations and all scattering modes. We also reveal the null-spaces for each scattering mode and verify those null-spaces analytically using a numerical singular value decomposition (SVD) analysis. In Section 5, we summarize the tradeoffs in two tables and show how three-component data can be utilized to determine all the orthorhombic parameters based on null-space intersections.

\section{Scattering radiation patterns of elastic parameters}

We give a brief history of patterns and then describe them mathematically. We start the mathematical description with the scattering function introduced by (Eaton \& Stewart, 1994), which describes the scattering of arbitrary plane waves on a point scatterer, and we discuss its reduction to diffraction-based (R.-S. Wu \& Aki, 1985 ; Tarantola, 1986) and reflection-based (Gholami et al., 2013a ; Alkhalifah \& Plessix, 2014) scattering radiation

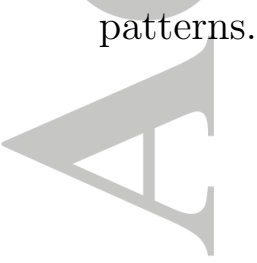

(C)2019 American Geophysical Union. All Rights Reserved. 


\subsection{Brief history of scattering radiation patterns}

We consider a multiparameter inverse problem with perfect illumination, where a small anisotropic scatterer is illuminated from all directions. We do not know the scatterer, but we have recorded a certain scattered wave mode, e.g., $P-P$ scattered waves. The function that depends on incident and scattered wavefields directions and describes the ratio between scattered and incident wavefield amplitudes is the scattering radiation pattern (R.-S. Wu \& Aki, 1985 ; Alkhalifah \& Plessix, 2014). Using these radiation patterns,

which are known from the recorded wave mode, we try to infer the elastic parameters of the scatterer.

Different types of radiation patterns indicate different types of elastic parameter perturbations and, therefore, can help to distinguish among the parameters from seismic data. (R.-S. Wu \& Aki, 1985) described radiation patterns for isotropic elastic parameters in the standard $(\lambda, \mu, \rho)$ parameterization. (Tarantola, 1986) considered scattering in several parameterizations of isotropic elastic media. He came to the conclusion that a parameterization featuring density and both $P$ and $S$ wave impedances was the most promising for reflection seismic data. (Eaton \& Stewart, 1994) introduced a scattering function that described the scattering of arbitrary plane waves on an arbitrary scatterer in background media with arbitrary anisotropy. (De Hoop et al., 1999) focused on the resolvable parameters in orthorhombic media and were probably the first to implicitly use reflection-based radiation patterns (Gholami et al., 2013a ; Alkhalifah \& Plessix, 2014 ; Kamath \& Tsvankin, 2016 ; He \& Plessix, 2016). (J.-W. Oh \& Alkhalifah, 2016) analyzed scattering for several parameterizations of orthorhombic media and proposed a hierarchi-

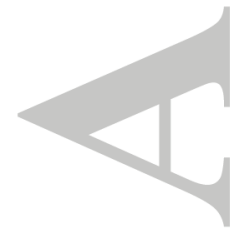

(C)2019 American Geophysical Union. All Rights Reserved. 
cal parameterization, which we use throughout this paper, to reduce the tradeoffs between parameters.

\subsection{Scattering angle - resolution length relation}

While radiation patterns characterize scattering for point perturbations, they can be generalized to arbitrary perturbations that fit into the Born approximation. If the scattering experiment is handled in the model wavenumber domain (Devaney, 1984), then we can access additional features of the scattering such as resolution (Fig. 2). For acoustic monochromatic plane waves, there is a simple relation between the wavenumbers in the model and the scattering angles (Ewald, 1969 ; Devaney, 1984). (R. Wu \& Toksöz, 1987) compared wavenumbers that are covered by scattered waves in vertical seismic profiling (VSP) and surface observations. (Mora, 1989) analyzed the contributions of reflections to the illuminated wavenumber spectrum in background media. (Kazei, Troyan, Kashtan, \& Mulder, 2013) provided a quantitative wavenumber illumination analysis of the spectrum covered by head waves and diving waves. (Podgornova, Leaney, Liang, \& others, 2015 ; Podgornova, Leaney, \& Liang, 2018) analyzed the resolvability of elastic parameter spectra in elastic VTI media. (Kazei \& Alkhalifah, 2018) presented spectral coverage and resolution for orthorhombic parameters.

In our previous work (Kazei \& Alkhalifah, 2018), we performed an analysis of the radiation patterns describing the scattering of $P$ waves on orthorhombic parameters in an isotropic, homogeneous-medium background. We present a similar analysis for different types of scattering. Namely, we consider $S$ waves and $P-S$ conversions. In order to access the seattering caused by isotropic and VTI parameter perturbations, we use a hierarchical

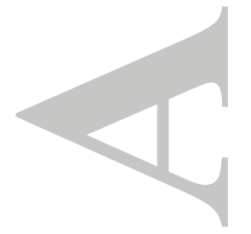

(C)2019 American Geophysical Union. All Rights Reserved. 
parameterization based on that of (J.-W. Oh \& Alkhalifah, 2016 ; Kazei \& Alkhalifah, 2018).

\subsection{Scattering function}

A monochromatic plane wave is defined by its phase, polarization, and propagation directions. The phase accumulated while traveling through a background medium is dropped in our analysis so that we can focus on the local scattering features. Therefore, here the scattered wavefield $\delta \mathbf{U}$ depends only on the incident plane-wave slowness vector $\mathbf{s}$, the scattered plane-wave slowness vector $\mathbf{g}$, the respective polarizations $\boldsymbol{\varsigma}$ and $\boldsymbol{\xi}$, the frequency $\omega$, and the perturbation of the parameters $\delta \mathbf{m}=(\delta \rho(\mathbf{x}), \delta \mathbf{c}(\mathbf{x}))^{T}$ :

$\delta \mathbf{U} \cdot \boldsymbol{\xi} \propto R_{\delta \mathbf{m}}(\mathbf{s}, \mathbf{g}, \boldsymbol{\varsigma}, \boldsymbol{\xi})$.

The coefficient $R$, well known for its arbitrary point scatterers, is called the scattering function (Eaton \& Stewart, 1994 ; R. K. Shaw \& Sen, 2004 ; Calvet, Chevrot, \& Souriau, 2006 ; Kazei \& Alkhalifah, 2018), and is defined as

$$
\begin{aligned}
R_{\delta \mathbf{m}}(\mathbf{s}, \mathbf{g}, \boldsymbol{\varsigma}, \boldsymbol{\xi}) & \equiv \varsigma_{k} \xi_{k} \delta \rho+s_{i} \varsigma_{j} \delta c_{i j k l} g_{k} \xi_{l} \\
& =\boldsymbol{\varsigma} \cdot \boldsymbol{\xi} \delta \rho+\mathbf{s} \boldsymbol{s}: \delta \mathbf{c}: \mathbf{g} \boldsymbol{\xi}
\end{aligned}
$$

While $R$ is independent of the background medium, which can have up to triclinicanisotropy complexity, eight different angles determine the directions of polarization and propagation for its incident and scattered wavefield (there are four directions, featuring two angles each). Thus, there are many possibilities for polarization and propagation relations in a general anisotropic background as well as for velocities of incident and scattered waves; here, we consider scattering in an isotropic background.

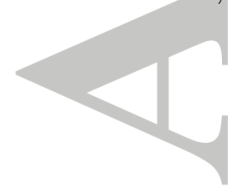

(C)2019 American Geophysical Union. All Rights Reserved. 


\subsection{Classic diffraction-based scattering radiation pattern $-\mathcal{D}$}

A general anisotropic medium allows for nine types of plane-wave scattering, each characterized by four independent angles that represent directions of propagation. For a given incident and scattered wave type in an isotropic background (e.g., $P-P, P-S V, S V-$

$S V, S V-S H$, etc.), the scattered wavefield becomes a function of the two vectors $\mathbf{s}$ and g. This function is the diffraction-based radiation pattern:

$\mathcal{D}_{W I-W S, \delta \mathbf{m}}(\mathbf{s}, \mathbf{g}) \equiv \frac{R(\mathbf{s}, \mathbf{g}, \boldsymbol{\varsigma}(\mathbf{s}, W I), \boldsymbol{\xi}(\mathbf{g}, W S), \delta \mathbf{m})}{\|\delta \mathbf{m}\|}$,

where $W I$ denotes the type of incident waves, and $W S$ denotes the type of scattered waves $\left(P-P, P-S_{i}, S_{i}-P\right.$, or $\left.S_{i}-S_{j}\right)$ for a given parameter perturbation $\delta \mathbf{m}$.

Diffraction-based radiation patterns characterize the scattering of certain types of plane waves on a point-like perturbation of the parameters. For example, in the case of $P-P$

$(W I=W S=P)$ scattering in an isotropic background

$$
\boldsymbol{\varsigma}(\mathbf{s}, W I-W S, \delta \mathbf{m})=\mathbf{s}, \boldsymbol{\xi}(\mathbf{g}, W I-W S, \delta \mathbf{m})=\mathbf{g}
$$

on a $\lambda$ perturbation,

$$
\begin{gathered}
\delta c_{i j k l}=\delta_{i j} \delta_{k l} \delta \lambda, W I-W S=P-P, \delta \rho=0, \delta m=\delta \lambda \\
\mathcal{D}_{W I-W S, \delta \mathbf{m}}(\mathbf{s}, \mathbf{g})=\mathcal{D}_{P-P, \delta \lambda}(\mathbf{s}, \mathbf{g})=\frac{1}{V_{p}^{2}}
\end{gathered}
$$

For vertically propagating incident $P$ waves, the scattering radiation patterns become functions of two real arguments, which are $\mathbf{g}$ vector components, and can be presented as a 2D plot (Eaton \& Stewart, 1994).

Diffraction-based radiation patterns were examined by (R.-S. Wu \& Aki, 1985 ; Tarantola, 1986 ; Beylkin \& Burridge, 1990) for isotropic parameters, and by (Calvet et al.,

2006) for VTI parameters in the classic (Thomsen, 1986) parameterization. For isotropic (C) 2019 American Geophysical Union. All Rights Reserved. 
parameters in an isotropic background, the full scattering information can be recovered by a single diffraction-based radiation pattern, as the only important parameter in that case is the difference between the incident and scattered directions (R.-S. Wu \& Aki, 1985 ; Tarantola, 1986). This is not the case in anisotropic media (Eaton \& Stewart, 1994 ; Calvet et al., 2006 ; J.-W. Oh \& Alkhalifah, 2016). We illustrate the dependence of the invoked source at the scatter location on the incidence using the example of the $C_{55}$ and $C_{12}$ elements shown in Fig. 3. The dependence of the diffraction patterns on the four angles describing the source and receiver directions complicates further analysis. To complete our analysis, we must reduce the diffraction-based patterns to reflection and transmission scattering radiation patterns.

\subsection{Reflection-based radiation patterns $-\mathcal{R}$}

Classic diffraction-based radiation patterns describe diffractions from point scatterers. However, seismic surveys are commonly dominated by reflections (Fig. 1(a)). Therefore, linearized reflection coefficients (Rüger, 1997) and, more recently, reflection-based radiation patterns (R. K. Shaw \& Sen, 2004 ; Gholami et al., 2013a ; Alkhalifah \& Plessix, 2014) were developed. A given wave-mode relation between the reflection-based radiation patterns and the reflection coefficients can be reduced to a scattering angle-dependent coefficient, which is independent of the scatterer type (R. K. Shaw \& Sen, 2004). In 3D scattering experiments, scattering functions depend on four scalar parameters, which are defined by unit vectors $\mathbf{s}$ and $\mathbf{g}$. For a plane reflector, the scattered wave direction is determined by the incident direction through Snell's law, and vice versa. Similarly, the dip and azimuth of the reflector determine how vector $\mathbf{s}$ is tied to vector $\mathbf{g}$ through Snell's

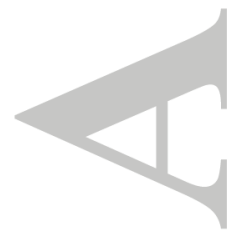

(C)2019 American Geophysical Union. All Rights Reserved. 
law, reducing the number of real variables that determine scattering from four to two:

$$
\mathcal{R}_{W I-W S, \delta \mathbf{m}}(\mathbf{g}) \equiv \mathcal{D}_{W I-W S, \delta \mathbf{m}}(\mathbf{s}(\mathbf{g}), \mathbf{g})
$$

The amplitude of the reflections from perturbations of isotropic parameters does not depend on the reflector orientation; therefore, the reflection-based radiation patterns are the same for all reflector orientations. For anisotropic reflectors and backgrounds, the reflection-based radiation patterns depend on the dip and azimuth angles. In most seismic cases, the medium is dominated by horizontal layering. For this reason, we follow the example of (Gholami et al., 2013a) and (Alkhalifah \& Plessix, 2014), and focus on scattering from horizontal reflectors. We illustrate typical reflection-based radiation patterns for $C_{i j}$ parameters in Fig. 7 .

\section{Wavenumber illumination theory}

In the previous section, we discussed the scattering of plane waves on point scatterers. But reflection-based radiation patterns can be remapped into the frequency-wavenumber domain in order to accommodate the scattering effects of any arbitrary perturbations that fit into the Born approximation. (Ewald, 1969) spheres have been used to recognize the relation between illuminated wavenumbers in a perturbation and the directions of its incident and scattered waves in monochromatic plane-wave experiments. For example, (Devaney, 1984) used this method to quantify the resolution of geophysical diffraction tomography images in the wavenumber domain. This technique was later applied to acoustic FWI scenarios for surface seismic acquisition (Mora, 1989) and vertical seismic profiling (R. Wu \& Toksöz, 1987). (Kazei, Troyan, et al., 2013) introduced the amplitude of the spectral sensitivity of the data to the perturbations of various background media,

(C)2019 American Geophysical Union. All Rights Reserved. 
and applied it to models with diving waves and multiples (Kazei, Kashtan, Troyan, \& Mulder, 2015, 2013). An elastic isotropic-background case with VTI perturbations was considered by (Podgornova et al., 2015). We show the applicability of this technique to arbitrary anisotropic perturbations of isotropic media.

In this section, we provide a derivation of the scattering function and generalize it to spectral sensitivities (Kazei, Troyan, et al., 2013 ; Podgornova et al., 2015 ; Kazei \& Alkhalifah, 2017). By incorporating the spectral sensitivities, we can consider a broad class of anomalies so small that we can neglect the second-order scattering.

\subsection{Far-field plane-wave scattering}

Radiation patterns simply represent different types of scattered wavefields for a point scatterer when the far-field or plane-wave approximation is applied to equation (9). While the derivation is well-known, we return to it in order to generalize the radiation patterns to arbitrary scatterers that satisfy equation (9). First, we utilize the approximation from the receiver side of equation (9), since it includes Green's tensors and not incident wavefields. Then, we treat the source wavefields in the same manner.

\subsubsection{The Born approximation}

We consider an incident wavefield $\mathbf{U}^{0}$ on a perturbation of the density $(\delta \rho(\mathbf{x}))$ and stiffness tensor $\left(\delta c_{k j p q}(\mathbf{x})\right)$ parameters. The frequency-domain Born approximation for the scattered wavefield $\delta \mathbf{U}$ in an anisotropic elastic medium is (Hudson \& Heritage, 1981 ; Beylkin \& Burridge, 1990 ; R. K. Shaw \& Sen, 2004)

$$
\delta U_{i}\left(\mathbf{x}_{s}, \mathbf{x}_{\mathbf{g}}, \omega\right)=\int_{V}\left(\delta \rho(\mathbf{x}) U_{k}^{0} \omega^{2}-\delta c_{k j p q}(\mathbf{x}) \frac{\partial U_{p}^{0}}{\partial x_{q}} \frac{\partial}{\partial x_{j}}\right) G_{i k}\left(\mathbf{x}_{g}, \mathbf{x}\right) \mathrm{d} \mathbf{x}
$$

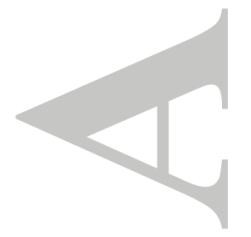

(C)2019 American Geophysical Union. All Rights Reserved. 
where $\omega$ is the angular frequency, $\mathbf{x}_{s}$ and $\mathbf{x}_{g}$ are the coordinates of the source and receiver, respectively, and $G_{i k}\left(\mathbf{x}_{g}, \mathbf{x}\right)$ is the Green's tensor for the background medium. Integration is performed over the volume of the perturbation $\delta \mathbf{m}=\left(\delta \rho, \delta c_{i j p q}\right)$, where $\mathbf{m}$ is the vector of unperturbed parameters. We can vectorize equation (9),

$$
\delta \mathbf{U}\left(\mathbf{x}_{s}, \mathbf{x}_{\mathbf{g}}, \omega\right)=\int_{V}\left(\omega^{2} \delta \rho(\mathbf{x}) \mathbf{U}^{0}(\mathbf{x}) \cdot \mathbf{G}(\mathbf{x})-\nabla \mathbf{G}(\mathbf{x}): \delta \mathbf{c}(\mathbf{x}):\left(\nabla \mathbf{U}^{0}(\mathbf{x})\right) \mathrm{d} \mathbf{x},\right.
$$

which already gives us some insight into the scattering. For example, scattering on the density perturbation is determined by the kernel

$$
\omega^{2} \delta \rho(\mathbf{x}) \mathbf{U}^{0}(\mathbf{x}) \cdot \mathbf{G}(\mathbf{x})
$$

and, hence, depends only on the amplitudes and polarization of the incident $\mathbf{U}^{0}$ and scattered $\mathbf{G}$ wavefields. For instance, if we consider the scattering of $S H$ waves in a vertical plane from a perturbation of density, then the amplitude of the scattered wavefield will be constant. At the same time, the scattering from perturbations of elastic constants $C_{i j}$, which are driven by the kernel

$\nabla \mathbf{G}(\mathbf{x}): \delta \mathbf{c}(\mathbf{x}):\left(\nabla \mathbf{U}^{0}(\mathbf{x})\right)$

can also depend on the propagation directions of the incident and scattered wavefields, which are extracted by the gradient $\nabla$ operator.

\subsubsection{Wavefields scattered by perturbation}

The Green's tensor $G_{i k}$ in the Born approximation (10) physically takes care of the propagation of a scattered wavefield from the perturbation to the receiver. The farfield approximation for the Green's tensor (Snieder, 2002) in a homogeneous, isotropic background consists of $P, S V$, and $S H$ waves:

$\mathbf{G}\left(\mathbf{x}_{g}, \mathbf{x}\right)=\alpha\left(\mathbf{G}_{P}\left(\mathbf{x}_{g}, \mathbf{x}(\mathbf{C})(\mathbf{G}, \mathrm{A}\right.\right.$ 
While $S V$ and $S H$ waves in isotropic media most often come together, they are usually recorded separately and therefore can be analyzed separately too. The coefficient

$$
\alpha=\frac{1}{4 \pi \rho V_{p}^{2}\left\|\mathbf{x}-\mathbf{x}_{g}\right\|}
$$

is dropped in our resolution analysis, since it is related to geometrical spreading and can be effectively compensated using pseudo-hessian techniques. Different components of the Green's function are approximated locally by plane waves. For the first component $\mathbf{G}_{P}$, the approximation leads to

$\mathbf{G}_{P}=e^{i \frac{\omega}{V_{p}} \mid \mathbf{x}_{g}-\mathbf{x} \|} \overline{\mathbf{g}} \overline{\mathbf{g}} \simeq e^{i \frac{\omega}{V_{p}} \mathbf{g} \cdot\left(\mathbf{x}-\mathbf{x}_{g}\right)} \overline{\mathbf{g}} \overline{\mathbf{g}}, \overline{\mathbf{g}}=\frac{\mathbf{g}}{|\mathbf{g}|}=\frac{\mathbf{x}-\mathbf{x}_{g}}{\left|\mathbf{x}-\mathbf{x}_{g}\right|}$

which is the $P$ wave approximated by a plane wave at the location $\mathbf{x} . \quad(\mathbf{g g})_{i k}=g_{i} g_{k}$ denotes standard outer product of vector $\mathbf{g}$ with itself. The second component, $\mathbf{G}_{S V}$, represents a shear wave polarized in the vertical plane that contains $\mathbf{x}$ and $\mathbf{x}_{g}$ :

$$
\mathbf{G}_{S V}=\frac{1}{\varkappa^{2}} e^{i \frac{\omega}{V_{s}}\left\|\mathbf{x}-\mathbf{x}_{g}\right\|} \mathbf{g}_{\theta} \mathbf{g}_{\theta} \simeq \frac{1}{\varkappa^{2}} e^{i \frac{\omega}{V_{s}} \mathbf{g} \cdot\left(\mathbf{x}-\mathbf{x}_{g}\right)} \mathbf{g}_{\theta} \mathbf{g}_{\theta}, \mathbf{g}_{\theta}=\left(\frac{\mathbf{g} \times \mathbf{e}_{z}}{\left|\mathbf{g} \times \mathbf{e}_{z}\right|} \times \overline{\mathbf{g}}\right)
$$

Finally, the third component of the Green's tensor, $\mathbf{G}_{S H}$, is an $S H$ wave polarized along

$$
\mathbf{g}_{\varphi}:
$$

$$
\mathbf{G}_{S H}=\frac{1}{\varkappa^{2}} e^{i \frac{\omega}{V_{s}}\left\|\mathbf{x}-\mathbf{x}_{g}\right\|} \mathbf{g}_{\varphi} \mathbf{g}_{\varphi} \simeq \frac{1}{\varkappa^{2}} e^{i \frac{\omega}{V_{s}} \mathbf{g} \cdot\left(\mathbf{x}-\mathbf{x}_{g}\right)} \mathbf{g}_{\varphi} \mathbf{g}_{\varphi}, \mathbf{g}_{\varphi}=\left(\frac{\mathbf{g} \times \mathbf{e}_{z}}{\left|\mathbf{g} \times \mathbf{e}_{z}\right|}\right),
$$

which is the horizontal component of a shear wave.

For the incident wavefield we consider point forces in three directions $\overline{\mathbf{s}}, \mathbf{s}_{\varphi}, \mathbf{s}_{\theta}$, leading to three respective wavetypes:

$$
\mathbf{U}_{0 P}=\mathbf{G}_{P}\left(\mathbf{x}_{s}, \mathbf{x}\right) \cdot \overline{\mathbf{s}},
$$

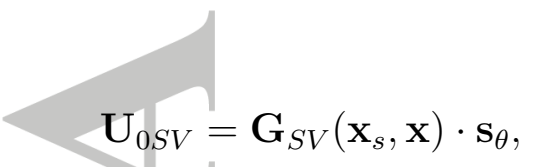

(C)2019 American Geophysical Union. All Rights Reserved. 


$$
\mathbf{U}_{0 S H}=\mathbf{G}_{S H}\left(\mathbf{x}_{s}, \mathbf{x}\right) \cdot \mathbf{s}_{\varphi} .
$$

\subsection{Model wavenumbers and scattering modes}

We start with scattered $P$ waves as they are the easiest to handle and by far the most popular in FWI applications. By substituting equations (18) and (15) into equation (9), and projecting the scattered wavefield $\mathbf{U}$ onto the direction of $\mathbf{g}$ (from the perturbation to the receiver), we obtain the approximate amplitude of the scattered $P-P$ waves (Kazei \& Alkhalifah, 2018) as

$$
\begin{aligned}
& \delta U_{P P}=\delta \mathbf{U}_{P} \cdot \overline{\mathbf{s}} \propto \int_{V} e^{i \mathbf{K} \cdot \mathbf{x}}(\overline{\mathbf{s}} \cdot \overline{\mathbf{g}} \delta \bar{\rho}(\mathbf{x})+\overline{\mathbf{s}} \overline{\mathbf{s}}: \delta \overline{\mathbf{c}}(\mathbf{x}): \overline{\mathbf{g}} \overline{\mathbf{g}}) \mathrm{d} \mathbf{x}, \\
& \text { where } \\
& \mathbf{K}=\omega(\mathbf{s}+\mathbf{g}) .
\end{aligned}
$$

Analogously, for the amplitude of the scattered $P-S V$ waves, we use equations (18) and (16), and project (9) onto vector $\mathbf{g}_{\theta}$ to obtain

$$
\delta U_{P S Y}=\delta \mathbf{U}_{P} \cdot \mathbf{s}_{\theta} \propto \int_{V} e^{i \mathbf{K}_{P S} \cdot \mathbf{x}}\left(\overline{\mathbf{s}} \cdot \mathbf{g}_{\theta} \delta \bar{\rho}+\frac{1}{\varkappa} \overline{\mathbf{s}} \overline{\mathbf{s}}: \delta \overline{\mathbf{c}}: \overline{\mathbf{g}} \mathbf{g}_{\theta}\right) \mathrm{d} \mathbf{x} .
$$

Replacing the polarization vector $\mathbf{g}_{\theta}$ in (39) with $\mathbf{g}_{\varphi}$ is sufficient to obtain similar expressions for the $P-S H$ scattering scenario. For $S$ waves, we obtain similar expressions by using equations (18), (17), and (9):

$$
\delta U_{S H S H}=\delta \mathbf{U}_{S H} \cdot \mathbf{s}_{\varphi} \propto \int_{V} e^{i \mathbf{K}_{S S} \cdot \mathbf{x}}\left(\mathbf{s}_{\varphi} \cdot \mathbf{g}_{\varphi} \delta \rho+\frac{1}{\varkappa^{2}} \overline{\mathbf{s}} \mathbf{s}_{\varphi}: \delta \overline{\mathbf{c}}: \overline{\mathbf{g}} \mathbf{g}_{\varphi}\right) \mathrm{d} \mathbf{x} .
$$

For different types of scattering, our further resolution analysis is based on equations (21-24) and their analogs, which describe the monochromatic scattering of $P$ waves.

Different scattering modes contribute to different wavenumbers at the same reflection

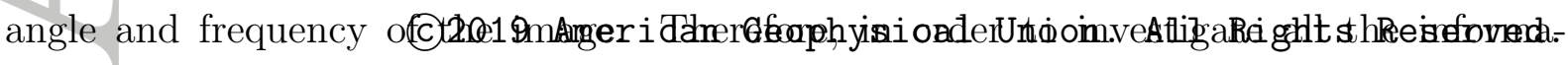


tion available, we need to remap the reflection-based radiation patterns into the spatial wavenumber domain. Equation (22) governs the mapping procedure and Fig. 5 illustrates how it defines the resolution of the different types of body wave scattering.

We note that the right-hand sides of equations (21), (23), and (24) are essentially Fourier transforms of the perturbation. Thus, we can generalize these equations as

$$
\delta U_{W I-W S}(\mathbf{s}, \mathbf{g}, \omega) \propto \boldsymbol{\varsigma} \cdot \boldsymbol{\xi} \delta \hat{\rho}\left(\mathbf{K}_{W I-W S}(\mathbf{s}, \mathbf{g}, \omega)\right)+\mathbf{s} \boldsymbol{s}: \delta \hat{\mathbf{c}}\left(\mathbf{K}_{W I-W S}(\mathbf{s}, \mathbf{g}, \omega)\right): \mathbf{g} \boldsymbol{\xi}
$$

Equation (25) shows that for a given scattering mode, the wavenumber resolved by inversion depends only on the frequency, source and receiver directions. On the other hand, we can infer all possible directions towards the source and receiver for a given wavenumber. Analyzing scattering for these directions leads directly to irresolvable tradeoffs in inversion at a particular length.

\subsection{Spectral sensitivities}

Vertical variations are often dominant in subsurface structures. Therefore, we focus on the vertical wavenumbers $\mathbf{K}_{W I-W S}$ in the perturbation spectrum. Given the scattering mode, azimuth, signal frequency, and wavenumber, equation (22) can be solved for the source and receiver directions $\mathbf{s}$ and $\mathbf{g}$, respectively (explicit equations for $\mathbf{s}$ and $\mathbf{g}$ are provided in appendix D). This means that the wavenumber defines the particular source and receiver directions, which are necessary to illuminate, for a single frequency and single scattering mode. In other words, the scattered data can be remapped into the model wavenumber and the azimuth $\varphi$ domain:

$$
\delta U_{W I-W S}(\mathbf{K}, \varphi, \omega) \equiv \delta U_{W I-W S}(\mathbf{s}(\mathbf{K}, \varphi), \mathbf{g}(\mathbf{K}, \varphi), \omega) \propto \delta \mathbf{m}\left(\mathbf{K}_{W I-W S}\right) .
$$

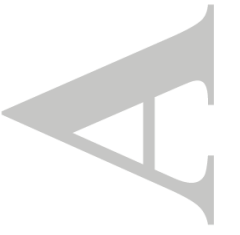

(C)2019 American Geophysical Union. All Rights Reserved. 
We call the coefficient of proportionality between the model and data shown in equation (26) the spectral sensitivity. The spectral sensitivity depends on the scattering parameter type, the normalized vertical wavenumber, and the azimuth, and determines both how the data represents the scattering from a particular parameter and which scales of the parameter are retrievable.

We formally define the spectral sensitivities by remapping the reflection-based radiation patterns into the azimuth-wavenumber domain. Then, we demonstrate how to read the spectral sensitivity maps on examples of scattering from a density perturbation, which is an isotropic parameter. As an additional example, we show scattering from a perturbation in $C_{55}$, which is an orthorhombic parameter.

\subsubsection{Definition}

We construct the sensitivities for the vertical wavenumbers $\mathbf{K}=\left(0,0, K_{z}\right)^{T}$. For a given frequency $\omega$ and a particular scattering mode, we find the corresponding source and receiver directions and remap the radiation patterns into the normalized wavenumberangle domain using

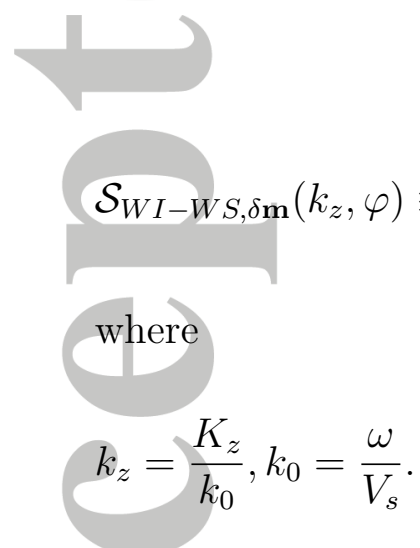

Equation (27) defines the spectral sensitivity for parameter $\delta \mathbf{m}$. We normalize all the wavenumbers, dividing them by $\omega / V_{s}$. These remapped patterns are functions of two variables $\left(k_{z}, \varphi\right)$ and, therefore, can be easily plotted and examined. We illustrate how (C)2019 American Geophysical Union. All Rights Reserved. 
the reflection patterns are mapped into the wavenumber domain, starting with the spectral sensitivities for density in Fig. 6.

\subsubsection{Examples}

Density is an isotropic parameter; the scattering does not vary with the azimuth in any of the scattering cases. In the $P-P$ scattering mode, the intermediate wavenumbers of density are not illuminated (white line in the middle of Fig. 6(b)), which leads to a poor reconstruction of the density in acoustic FWI (Mulder \& Plessix, 2008). $P-S V$ waves illuminate the missing intermediate wavenumbers in the density perturbations (Fig 6.(b)) and can help reconstruct it. $S H$ waves are difficult to emit in seismic exploration, but they evenly illuminate the full density spectrum (Fig. 6(d)).

For anisotropic parameters scattering can depend on the azimuth of the incident and scattered wavefields. The reflection-based radiation pattern for $C_{55}$ and $P-P$ scattering is represented in Fig. 7(a). This mapping allows us to compare wavenumbers and resolution covered by different wave types. In the next section, we analyze the scattering of all the elastic constants and find the null-space of the inversion for each scattering mode.

\section{Spectral sensitivity matrices and numerical analysis of tradeoffs}

In the case of an isotropic background and a general anisotropic scatterer, there are six combinations of incident-scattered wave types and 22 elastic parameters to perturb. Thus, the total number of radiation patterns specified by $W I-W S, \delta \mathbf{m}$ is $22^{*} 6=132$, and this is the amount of data we need to analyze triclinic perturbations. In this section, we present the spectral sensitivities for all these parameters and discuss some features of the scattering modes case-by-case in the subsections.

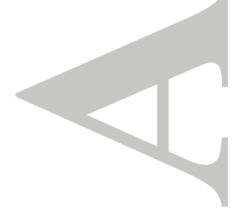

(C)2019 American Geophysical Union. All Rights Reserved. 
For the orthorhombic and VTI parameters, we find all the tradeoffs numerically with SVD and confirm them analytically. Our SVD analysis of the radiation patterns answers the following questions:

1. What is the null-space of the linearized dynamic inversions?

2. How many orthorhombic parameters can be resolved from a given scattering mode? Of course, the answers to these questions depend on the method of acquisition and the signal-to-noise ratio of the data, assuming that coverage is perfect and there is no noise. Therefore, the tradeoffs we find are irresolvable.

\subsection{Sensitivity matrices $\mathbb{S}_{W I-W S}-$ assembly}

In this subsection, we discuss the matrix form of the simplified inversion problem for the vertical wavenumber $\mathbf{K}$ from arbitrary scattering mode. For an arbitrary perturbation of parameters $\mathbf{m}(\mathbf{K})$, the scattered wavefield can be expressed in the wavenumber domain

as

$\delta U_{W I-W S}\left(k_{z}, \varphi, \omega\left(k_{z}, K_{z}\right)\right) \propto \mathbf{S}_{W I-W S}\left(k_{z}, \varphi\right) \delta \mathbf{m}(\mathbf{K})$

Vector $\mathbf{S}_{W I-W S}\left(k_{z}, \varphi\right)$ represents the set of reflection-based radiation patterns remapped into the normalized wavenumber domain. For example, element $S_{1}\left(k_{z}, \varphi\right) \equiv$ $\mathcal{S}_{W I-W S, \delta \bar{\rho}}\left(k_{z}, \varphi\right)$ is the radiation pattern of the density.

In matrix form, equation $(29)$ can be written for all $\left(k_{z}, \varphi\right)$ corresponding to a given $K_{z}$ together as follows:

$\delta \mathbf{U}_{W I-W S}\left(K_{z}\right) \propto \mathbb{S}_{W I-W S} \delta \mathbf{m}\left(K_{z}\right)$

Columns of the matrix $\mathbb{S}$ are, spectral sensitivities reshaped into vectors. Matrix $\mathbb{S}$ determines resolvability of parameters at a given spatial resolution. It's rank is equal to the (C)2019 American Geophysical Union. All Rights Reserved. 
maximum number of resolvable parameters. Matrix $\mathbb{S}$ can also be linked to the Hessian of standard full-waveform inversion (Kazei \& Alkhalifah, 2018 ; Podgornova et al., 2018).

\subsection{Hierarchical parameterization}

In order to obtain more insight into scattering from orthorhombic parameters and look at some scattering features for VTI and isotropic parameters we use parameterization introduced by (J.-W. Oh \& Alkhalifah, 2016). The parameterization splits ten orthorhombic parameters into three groups: isotropic, VTI, orthorhombic, so that if VTI and orthorhombic parameters are zero, the medium is isotropic and if orthorhombic parameters are zero, then the medium is VTI. Isotropic media is described by three non-zero parameters $V_{p}, V_{s}$, and $\rho$.

By adding three more parameters $-\varepsilon_{1}, \eta_{1}$, and $\gamma_{1}-$ we describe VTI media. This specific choice of parametrization for VTI reduces the trade offs for surface seismic data inversion objectives as shown by (Alkhalifah \& Plessix, 2014). In this parameterization $\epsilon$ describes the elliptic part of of anisotropy of $P$ waves. Parameter $\eta$ represents the anelliptic part of the anisotropy of $P$ waves. The $\gamma$ parameter describes the anisotropy of horizontally polarized $S H$ waves. In VTI medium $S H$ waves show elliptic anisotropy and therefore one parameter is sufficient to describe their kinematics. Hence we work with orthorhombic media we add 1 indexes to reflect that this parameters belong to the $x_{1}-x_{3}$ plane and the other vertical plane of symmetry $x_{2}-x_{3}$ may have different VTI behavior.

Finally, four additional parameters $-\varepsilon_{d}, \eta_{d}, \gamma_{d}$, and $\delta_{3}$ - allow us to go from VTI to orthorhombic anisotropy. As we mentioned before there are two vertical planes of symmetry in orthorhombic medium. Wave propagation in the first plane $x_{1}-x_{3}$ is fully defined by the above mentioned parameters, the plane $x_{2}-x_{3}$, on the other hand, can have different

(C)2019 American Geophysical Union. All Rights Reserved. 
parameters. Say those are $\varepsilon_{2}, \eta_{2}$, and $\gamma_{2}$, we can introduce the deviation parameters

$$
\begin{aligned}
\varepsilon_{d} & =\frac{\varepsilon_{2}-\varepsilon_{1}}{1+2 \varepsilon_{1}} \\
\eta_{d} & =\frac{\eta_{2}-\eta_{1}}{1+2 \eta_{1}} \\
\gamma_{d} & =\frac{\gamma_{2}-\gamma_{1}}{1+2 \gamma_{2}}
\end{aligned}
$$

that will tell us how different are the two vertical planes of symmetry of orthorhombic medium. Additional parameter $\delta_{3}$ determines the anellipticity of $P$ wave anisotropy in the $x_{1}-x_{2}$ plane (J.-W. Oh \& Alkhalifah, 2016). Explicit equations that can be used to derive the elastic stiffness matrix elements from the hierarchical parameters can be found in the Glossary.

Radiation patterns and the spectral sensitivities for any parameterization can be obtained through variable substitution to $C_{i j}, \rho$ parameterization.

$$
\boldsymbol{\mathcal { R }}_{\mathbf{m}, W T}=\boldsymbol{\mathcal { R }}_{\mathbf{m}_{0}(\mathbf{m}), W T}=\boldsymbol{\mathcal { R }}_{\frac{\partial \mathbf{m}_{0}}{\partial \mathbf{m}} \mathbf{m}}
$$

The Jacobian $\frac{\partial\left(\bar{C}_{i j}, \bar{\rho}\right)}{\partial \mathbf{m}}$ for the hierarchical parameterization has the following form:

$$
\left(\begin{array}{c}
\bar{\rho}_{1} \\
\bar{C}_{11} \\
\bar{C}_{22} \\
\bar{C}_{33} \\
\bar{C}_{12} \\
\bar{C}_{13} \\
\bar{C}_{23} \\
\bar{C}_{44} \\
\bar{C}_{55} \\
\bar{C}_{66}
\end{array}\right)=\left(\begin{array}{cccccccccc}
1 & 0 & 0 & 0 & 0 & 0 & 0 & 0 & 0 & 0 \\
1 & 2 & 0 & 0 & 0 & 0 & 0 & 0 & 0 & 0 \\
1 & 2 & 0 & 0 & 2 & 0 & 0 & 0 & 0 & 0 \\
1 & 2 & 0 & -2 & 0 & 0 & 0 & 0 & 0 & 0 \\
1-2 \varkappa & 2 & -4 \varkappa & 0 & 0 & 0 & 0 & 1 & -4 \varkappa & 0 \\
1-2 \varkappa & 2 & -4 \varkappa & -1 & 0 & -1 & 0 & 0 & 0 & 0 \\
1-2 \varkappa & 2 & -4 \varkappa & -1 & 1 & -1 & -1 & 0 & 0 & -4 \varkappa \\
\varkappa & 0 & 2 \varkappa & 0 & 0 & 0 & 0 & 0 & 0 & 2 \varkappa \\
\varkappa & 0 & 2 \varkappa & 0 & 0 & 0 & 0 & 0 & 0 & 0 \\
\varkappa & 0 & 2 \varkappa & 0 & 0 & 0 & 0 & 0 & 2 \varkappa & 0
\end{array}\right) \delta\left(\begin{array}{c}
\bar{\rho} \\
\bar{V}_{p} \\
\bar{V}_{s} \\
\varepsilon_{1} \\
\varepsilon_{d} \\
\eta_{1} \\
\eta_{d} \\
\delta_{3} \\
\gamma_{1} \\
\gamma_{d}
\end{array}\right)
$$

We also present the partial derivatives of the $C_{i j}$ parameters in a graph (Fig. 8) to illustrate the perturbations in the elastic parameters that are introduced by perturbations from a single hierarchical parameter. For example, perturbing $V_{p}$ is equivalent to simultaneously perturbing all $C_{i j}$ with $i \leq 3$ and $j \leq 3$ (see the second column in equation (35)); (C)2019 American Geophysical Union. All Rights Reserved. 
and perturbing $\gamma_{1}$ is equivalent to simultaneously perturbing $C_{12}$ and $C_{66}$ (see the ninth column in the matrix in equation (35)). To simplify formulas and focus on geometric properties of scattering we normalize units in the following way - velocities are normalized by the background $V_{p 0}$, densities by $\rho_{0}$, elastic constants by $\rho_{0} V_{p}^{2}$. The normalized quantities are distinguished by the dash, e.g.

$$
\bar{V}_{p} \equiv \frac{V_{p}}{V_{p 0}}, \bar{V}_{s} \equiv \frac{V_{s}}{V_{p 0}}, \bar{C}_{55} \equiv \frac{C_{55}}{\rho V_{p 0}^{2}}
$$

An advanced choice of parameterization will give some insights that are not straightforward from the $C_{i j}$ parameterization. For example, parameters contributing to $V_{p}$ provide zero sensitivity to $S$-wave scattering. Being a combination of nine $C_{i j}$ parameters, $V_{p}$ is hardly discoverable in the original parameterization. Thus, our knowledge is still limited by the choice of parameterization, as we cannot visually identify coupling among three or more parameters.

\section{3. $P-P$ scattering}

If registered, $P$ waves are the first waves that arrive at the receivers. Therefore, their travel times, or "first breaks", are the most common in seismic inversion setups. The finitefrequency $P$-wave travel times are well represented by effective pseudo-acoustic modeling (Alkhalifah, 2000 ; Z. Wu \& Alkhalifah, 2017), though correct inversion of the amplitudes requires elastic-media representation even in isotropic media (Raknes \& Arntsen, 2014 ; Kurzmann et al., 2016). In global seismology, the $P$ waves served as the main source of information about the core mantle and inner core-outer core boundaries for a long time (Bolt, 1970). $P$ waves are still extensively exploited in inversions for the earth's inner core (Peng, Koper, Vidale, Leyton, \& Shearer, 2008 ; Yu, 2016 ; Irving \& Deuss, 2015), 
as well as for inversions of the mantle and crust. In our previous work, we analyzed the scattering of $P$ wave-scattered data with respect to the inversion opportunities (Kazei \& Alkhalifah, 2018); therefore, here we cover the decoupling of parameters in the case of perfect illumination only.

In Fig. 9(a) we see four non-orthorhombic elements that show non-zero sensitivity. These non-zero sensitivities can potentially be used to invert for a monoclinic medium or an orthorhombic medium that is rotated around the vertical axis. On the other hand, the sensitivity to tilts - rotations around horizontal axis - is zero; therefore, inversion for tilts from monotypic waves will not work for purely vertical wavenumbers.

Through SVD of the $\mathbb{A}$ matrix, each row represents a particular scattering experiment. Data at all angles in the radiation patterns are inverted together. The number of parameters that are invertible is equal to the number of non-zero singular values in SVD. The principal null-space is the span of singular vectors related to zero singular values. The graph of the singular values Fig. 10(a) tells us how the condition number of the inverse problem decreases when we try to regularize inversion by reducing the number of parameters to be inverted.

We analyze the SVD of the full matrix $\mathbb{A}_{P-P}$ in the $C_{i j}, \rho$ parameterization. Fig. 10(a) shows that the matrix has only six non-zero singular values and, thus, only six independent parameters are invertible in this case. Fig. 10(b) shows the set of singular vectors in the $C_{i j}, \rho$ parameterization. The numerical null-space is defined as the linear span of four singular vectors, corresponding to zero singular values (columns 7-10 in Fig.

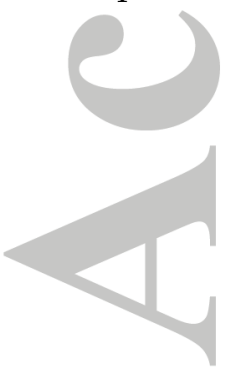

(C)2019 American Geophysical Union. All Rights Reserved. 
10(b)). These vectors are

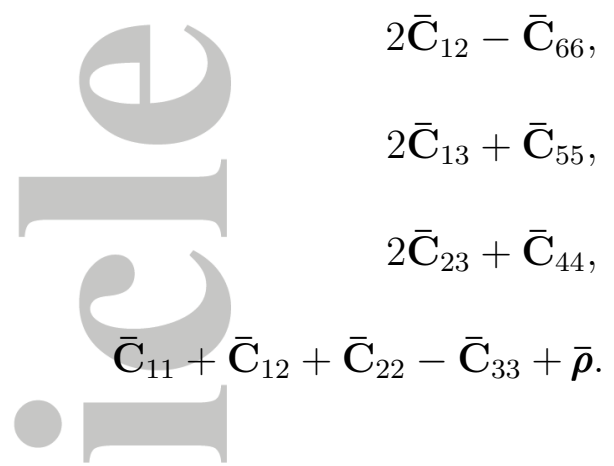

By substituting these combinations, which are found empirically, into the reflection-based radiation pattern expressions, (Kazei \& Alkhalifah, 2018) showed that the inversion is not at all sensitive to them. For example

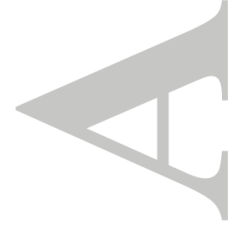

$$
\begin{aligned}
\mathcal{R}_{2 \overline{\mathbf{C}}_{12}-\overline{\mathbf{C}}_{66}, P-P} & \\
& =\mathcal{R}_{2 \mathbf{c}_{1122}-\mathbf{c}_{1212}-\mathbf{c}_{2121}-\mathbf{c}_{1221}-\mathbf{c}_{2112}, P-P}(\mathbf{s}, \mathbf{g}) \\
& =4 s_{1} s_{1} g_{2} g_{2}-4 s_{1} s_{2} g_{1} g_{2}=0
\end{aligned}
$$

We also found (Kazei \& Alkhalifah, 2018) a particular combination of the stiffness matrix elements and density that does not produce reflections. The reflection-based radiation

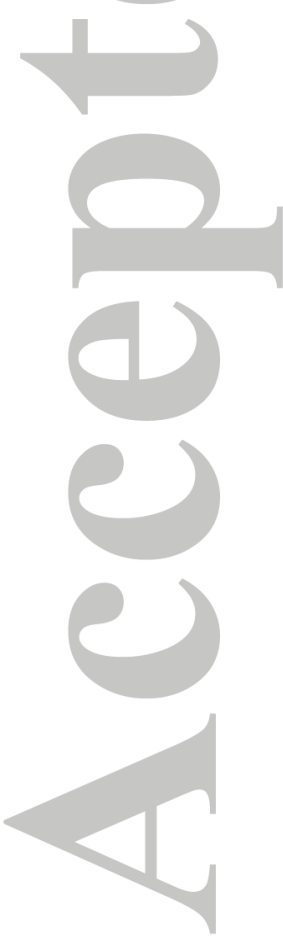

(C)2019 American Geophysical Union. All Rights Reserved. 
pattern of density can be transformed as follows:

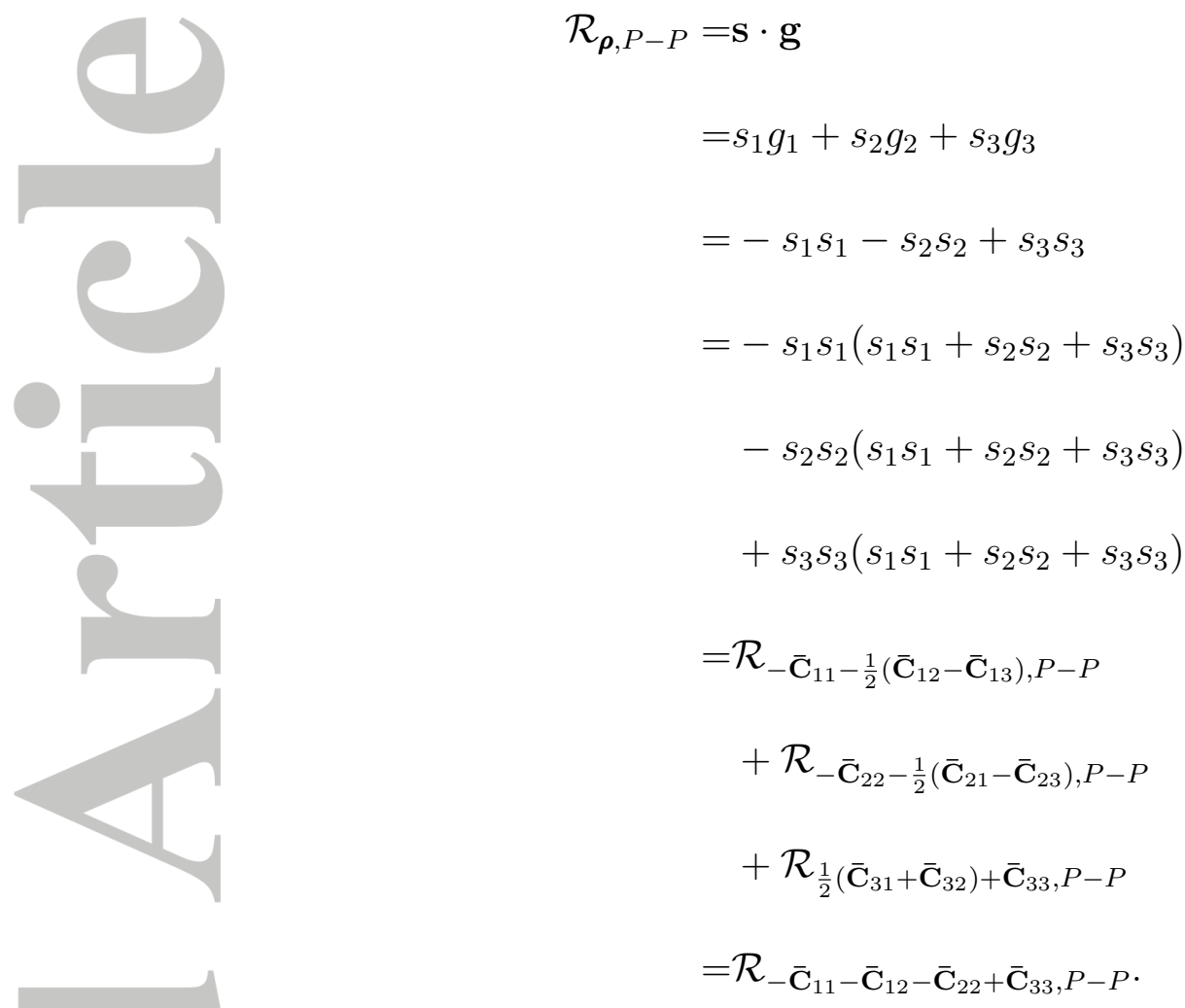

Thus, density can be effectively represented by anisotropic parameters in the first order of scattering. Therefore, it is impossible to reconstruct density simultaneously with all the VTI parameters when starting from isotropic background.

\section{4. $S H-S H$ scattering}

Shear-wave scattering at the core-mantle boundary revealed significant anisotropy (Fukao, 1984 ; Silver \& Chan, 1991 ; Babuska \& Cara, 1991 ; L. P. Vinnik, Makeyeva, Milev, \& Usenko, 1992). More recently, the existence of a beyond-VTI anisotropy was reconfirmed (Wookey, Kendall, \& Rümpker, 2005). Horizontally polarized shear waves and their superpositions propagating as Love waves had a significant impact on recent near-surface inversions (Pan, Xia, Xu, Gao, \& Xu, 2015 ; Dokter, Köhn, Wilken, De Nil, (C)2019 American Geophysical Union. All Rights Reserved. 
\& Rabbel, 2017). Typically, inversions focus on the velocity of $S H$ waves along the horizontal axis, below we investigate which tradeoffs are irresolvable in inversions of this particular mode. The scattering of $S H$ waves in the wavenumber domain is governed by the equation

$$
\delta U_{S H S H} \equiv \mathbf{s}_{\varphi} \cdot \mathbf{g}_{\varphi} \delta \hat{\rho}\left(\mathbf{K}_{S S}\right)+\mathbf{s s}_{\varphi}: \delta \hat{\mathbf{c}}\left(\mathbf{K}_{S S}\right): \mathbf{g g}_{\varphi} .
$$

The polarization of $S H$ waves is horizontal; therefore, $\mathbf{s}_{\varphi 3}=\mathbf{g}_{\varphi 3}=0$, which leads to zero sensitivity to the $C_{13}, C_{23}$, and $C_{33}$ parameters of $S H$ scattering (Fig. 11(a)). Also, $\varepsilon_{1}, \eta_{1}$, and $\eta_{d}$ span the same linear space as the $C_{13}, C_{23}$, and $C_{33}$ parameters, and all show zero sensitivity (Fig. 11(b)). The central parameter of $S H$ wave inversions is the horizontal shear velocity ( $V_{s}$ in the new parameterization), with $\gamma_{1}$ being responsible for the anisotropy between vertical and horizontal wave propagation. However, the scattering panels Fig. 11(b) show that $V_{s}$ is strongly coupled with density in a dynamic inversion.

Neither $V_{p}$ nor the equivalent $\lambda$ perturbation of the parameters scatter $S H$ waves (R.S. Wu \& Aki, 1985). Density shows a constant scattering amplitude according to equation (43), which is coupled to a combination of $C_{11}, C_{44}, C_{55}$, and $C_{66}$.

In the new parameterization, the non-scattering perturbation

$$
\rho-2 \mathbf{C}_{12}+\mathbf{C}_{66}-\mathbf{C}_{44}-\mathbf{C}_{55}
$$

is equivalent to

$$
2 \varkappa \rho-(1+\varkappa) \mathbf{V}_{s}+2 \gamma_{1}
$$

according to the partial derivative matrix $\frac{\partial \mathbf{C}}{\partial \mathbf{m}}$. Equations $(44,45)$ finalize the null-space of the inversion using $S H-S H$ waves.

(C)2019 American Geophysical Union. All Rights Reserved. 


\section{5. $S V-S V$ scattering}

The $S V-S V$ scattering mode is heavily utilized in global seismology in order to infer the anisotropy of the earth's deeper structures. In particular, diffracted $S V$ waves observed in the $S H$ shadow zone led to the discovery of possible anisotropy at the core-mantle boundary (L. P. Vinnik, Farra, \& Romanowicz, 1989 ; Babuska \& Cara, 1991 ; L. Vinnik, Romanowicz, Le Stunff, \& Makeyeva, 1995).

The scattering of an $S V$ wave from an incident $S V$ wave under the Born approximation is represented by the following formula:

$\delta U_{S V S V} \equiv \frac{1}{\varkappa^{3}} \mathbf{s}_{\theta} \cdot \mathbf{g}_{\varphi} \delta \hat{\rho}\left(\mathbf{K}_{S S}\right)+\mathbf{s s}_{\varphi}: \delta \hat{\mathbf{c}}\left(\mathbf{K}_{S S}\right): \mathbf{g g}_{\varphi}$

Just like $P-P$ waves, $S V-S V$ waves are only sensitive to monoclinic parameters (Fig. 13(a)). In the hierarchical parameterization, $V_{p}, \gamma_{1}$, and $\epsilon_{1}$ do not scatter these types of waves. Scattering by density $(\rho)$ resembles $V_{s}$, and scattering by anomalies in $\epsilon_{d}$ resemble $\delta_{3}$.

There are six non-zero singular values in this case (Fig. 14(a)); therefore, only six parameters can be inverted from the $S V-S V$ scattered waves. Density is not completely coupled to some parameters and can be recovered, as it is not present in the set of zero singular vectors (7-10) that span the null-space of inversion. In the hierarchical parameterization, the density strongly resembles $V_{s}$. Since $\mathcal{R}_{S V-S V, \delta_{3}}=-\mathcal{R}_{S V-S V, \varepsilon_{d}}$, the sum of $\varepsilon_{d}$ and $\delta_{3}$ does not scatter in the reflection mode $\mathcal{R}_{S V-S V, \varepsilon_{d}+\delta_{3}} \equiv 0$.

\section{6. $P-S V$ scattering}

$P-S V$ scattering is important in multi-component acquisition (e.g., ocean-bottom cables or nodes) and has proven useful in imaging and inversion near gas clouds (Brossier, (C)2019 American Geophysical Union. All Rights Reserved. 
Operto, \& Virieux, 2009 ; Prieux, Brossier, Operto, \& Virieux, 2013). P-SV scattering in the wavenumber domain is governed by the formula

$\delta U_{P S V}(\mathbf{s}, \mathbf{g}, \omega)=\frac{1}{\varkappa^{3}}\left(\mathbf{s} \cdot \mathbf{g}_{\theta} \delta \hat{\rho}\left(\mathbf{K}_{P S}\right)+\mathbf{s s}: \delta \hat{\mathbf{c}}\left(\mathbf{K}_{P S}\right): \mathbf{g g}_{\theta}\right)$

Among the orthorhombic parameters of $P-S V$ scattered waves, we observe that $C_{12}$ is coupled to $C_{66}$ (Fig. 14(a)), which results in no scattering by $\gamma_{1}$ in the hierarchical parameterization. At the same time, $V_{p}$ presents a non-scattering combination in the hierarchical parameterization. We also observe that all the parameters in the $C_{i j}$ parameterization are scattered, that $C_{14}$ is coupled to $C_{56}$, and that $C_{25}$ is coupled to $C_{46}$. SVD shows that there is no scattering on $V_{p}$ or $\gamma_{1}$ (Fig. 15(c)).

$P-S V$ waves show non-zero sensitivity for all the $C_{i j}$ parameters, but have zero sensitivity to low wavenumbers (Podgornova et al., 2015). However, they are key to inverting for tilted orthorhombic media (TORT).

\section{7. $P-S H$ scattering}

$P-S H$ scattering does not exist in VTI media or in slices of orthorhombic media aligned with the symmetry planes. Therefore, it can be used to find these symmetry planes in orthorhombic inversion. We expect the deviation parameter $\delta_{3}$ to play an important role in the sensitivity matrix. $P-S H$ scattering is governed by the formula

$$
\delta U_{P S H}(\mathbf{s}, \mathbf{g}, \omega)=\frac{1}{\varkappa^{3}}\left(\mathbf{s} \cdot \mathbf{g}_{\varphi} \delta \hat{\rho}\left(\mathbf{K}_{P S}\right)+\mathbf{s s}: \delta \hat{\mathbf{c}}\left(\mathbf{K}_{P S}\right): \mathbf{g g}_{\varphi}\right) .
$$

$P-S H$ waves are sensitive to all the $C_{i j}$ parameters in a classic $C_{i j}, \rho$ parameterization except $C_{33}$ and density. Therefore, it is almost impossible to distinguish coupling effects in the sensitivities shown in Fig. 17(a).

(C)2019 American Geophysical Union. All Rights Reserved. 
$P-S H$ scattering does not happen for VTI parameters $\left(\rho, V_{p}, V_{s}, \epsilon_{1}, \eta_{1}, \gamma_{1}\right)$ as there is no preferred direction of polarization for the scattered wave (Fig. 17(b)). Therefore, if observed, $P-S H$ conversion clearly indicates a beyond-VTI anisotropy. As our SVD analysis suggests, if the VTI parameters are known, then the rest of the orthorhombic parameters can be reconstructed from the $P-S H$ scattering mode alone (Fig. 18(a)).

\section{8. $S V-S H$ scattering}

This component is important in global inversions of scattering. The formula governing $S V-S H$ scattering is given by

$$
\delta U_{S V S H} \equiv \frac{1}{\varkappa^{3}} \int_{V} e^{i \mathbf{K}_{S S} \cdot \mathbf{x}}\left(\mathbf{s}_{\theta} \cdot \mathbf{g}_{\varphi} \delta \rho+\mathbf{s s}_{\theta}: \delta \mathbf{c}: \mathbf{g g}_{\varphi}\right) \mathrm{d} \mathbf{x} .
$$

$S V-S H$ scattering, like $P-S H$ scattering, cannot happen on VTI parameters since there is no preferred direction for the scattered $S H$ wave polarization. When the perturbation is anisotropic, however, $S V-S H$ and $P-S H$ scattering become possible. These types of scattering, if they happen, are clear indicators of azimuthal anisotropy. An additional tradeoff in this case is the coupling of $\epsilon_{d}$ and $\delta_{3}$ (Fig. 11(b)). We derive the exact relation between patterns of $\epsilon_{d}$ and $\delta_{3}$ :

$$
\begin{aligned}
& \text { (RS } \\
& \mathcal{R}_{S V-S H, \varepsilon_{d}}=\mathcal{R}_{S V-S H, 2 \mathbf{C}_{22}+\mathbf{C}_{23}}=s_{2} s_{\theta 2} g_{2} g_{\varphi 2}+s_{2} s_{\theta 2} g_{3} g_{\varphi 3}+s_{3} s_{\theta 3} g_{2} g_{\varphi 2}= \\
& s_{2} s_{\theta 2} g_{2} g_{\varphi 2}+s_{3} s_{\theta 3} g_{2} g_{\varphi 2}=\left(s_{2} s_{\theta 2}+s_{3} s_{\theta 3}\right) g_{2} g_{\varphi 2}= \\
& \left(\mathbf{s} \cdot \mathbf{s}_{\theta}-s_{1} s_{\theta 1}\right) g_{2} g_{\varphi 2}=-s_{1} s_{\theta 1} g_{2} g_{\varphi 2}=-\frac{1}{2} \mathcal{R}_{S V-S H, 2 \mathbf{C}_{12}}=-\frac{1}{2} \mathcal{R}_{S V-S H, \delta_{3}} .
\end{aligned}
$$

(C)2019 American Geophysical Union. All Rights Reserved. 


\section{Discussion}

Spectral sensitivity patterns fully characterize the Born linearized reflection of body waves on different scatterers and can be useful for inversion and acquisition design. Reflected body waves are the main source of information about deep targets inside the earth, such as oil reservoirs or the inner and outer core boundaries, though they are not the only waves that can be inverted in FWI. Also, Born scattering may be successfully applied in amplitude-versus-offset techniques for the characterization of oil reservoirs (Beretta, Bernasconi, \& Drufuca, 2002 ; R. Shaw \& Sen, 2006 ; den Boer \& Sayers, 2018) and in inversion of the anisotropic properties of the inner core (Vidale \& Earle, 2000). We note, however, that it is not always a valid approximation. In the following, we discuss how the scattering of other wave types can be accessed similarly to body waves, and then explain where the results of this work could be useful in the design of an anisotropic elastic FWI.

\subsection{Beyond body waves}

FWI optimizes the model to fit the full set of observed waveforms. We discussed only linearized scattering of body waves. Therefore, the nonlinear effects in wave propagation due to multiple or distributed perturbations in the background model, such as multiscattering, are left out of the scope of this paper. The linear scattering of multiples and surface or guided waves are also out of the scope of our analysis. Surface waves can be accessed similarly to body waves if the scattering functions are modified accordingly. Scattering functions for surface waves are sometimes called interaction matrices and can be found in (Snieder, 1986), and an analysis of their numerical kernels can be found in (Sieminski,

Liu, Trampert, \& Tromp, 2007). If they occur in the background medium, multiples can enhance the illumination of certain wavenumbers in the spectrum of the model perturba(C)2019 American Geophysical Union. All Rights Reserved. 
tion, leading to sinusoidal artifacts in FWI (Kazei, Kashtan, et al., 2013 ; Kazei et al., 2015). If the multiples are of a certain order and separated from other waves, then they can actually improve the resolution of the migration (Schuster \& Huang, 2014). Multiple scattered waves may also enhance the illumination of deeper parts of the model in FWI (Alkhalifah \& Plessix, 2014).

\subsection{Possible applications}

Multiparameter FWI is a challenging problem. The wavenumber illumination concept for 2D (Podgornova et al., 2018) and 3D (Kazei \& Alkhalifah, 2018) multiparameter FWI gives very important guidelines. In monoparameter FWI, we seek full wavenumber coverage (Devaney, 1984 ; Mora, 1989 ; Operto et al., 2015 ; Alkhalifah, 2016). In multiparameter FWI, we need to illuminate each model wavenumber at least as many times as the number of parameters we want to invert for. Scattering maps can give an idea of which parameters we are able to distinguish in FWI, depending on the body waves that are available. In 2D FWI, if we want to invert N parameters from the medium with single component data, then we must invert at least $\mathrm{N}$ frequencies simultaneously. In 3D FWI, only the azimuthal variations can be retrieved from single frequency, single component data. Of course, more parameters can be estimated if constraints are imposed on the spatial variations in the model. The interaction of the regularizations (Esser, Guasch, van Leeuwen, Aravkin, \& Herrmann, 2016 ; Kazei, Kalita, \& Alkhalifah, 2017 ; Kalita, Kazei, Choi, \& Alkhalifah, 2018) and the conditioning of the FWI updates (Alkhalifah \& Plessix, 2014 ; Ovcharenko, Kazei, Peter, \& Alkhalifah, 2018) require further investigation.

The choice of parameterization is critical for multiparameter FWI applications (Tarantola, 1986 ; Gholami, Brossier, Operto, Ribodetti, \& Virieux, 2013b ; Alkhali(C)2019 American Geophysical Union. All Rights Reserved. 
fah \& Plessix, 2014). Depending on the data that is available in a given setup, different anisotropic parameters may be chosen for inversion. In the case where reflections are inverted, we show that the null space will always limit the inversion results. Therefore, a good choice of parameters would isolate this null space via the parameterization. If the non-scattering combinations are isolated by the chosen parameters, then those parameters can be kept out of the inversion, which should improve the FWI convergence. If some parameters from the null space are inverted in FWI, we can expect higher uncertainty in those parameters.

In real datasets, noise and aperture further limit the capabilities of multiparameter FWI (Masmoudi \& Alkhalifah, 2018 ; J. Oh \& Alkhalifah, 2018). In this text, we presented graphs of the singular values, which can be used to limit the number of parameters inverted. Perturbations in other parameters of the same amplitude as the true model perturbation will lead to the wavefields having the same amplitude as noise. Therefore, one intuitive method of choosing the number of parameters $N$ is to take the maximum value of $N$ that satisfies the inequality $\left|S_{1} / S_{N}\right|>S N R$, where $S_{1}$ is the first and largest singular value, and $\left|S_{i+1}\right|<\left|S_{i}\right|$.

We note that SNR should be calculated for the FWI gradient and not the data itself. Ideally, the gap $S_{N}-S_{N+1}$ should be maximized to stabilize the inversion (Cheverda \& Kostin, 1995 ; Kazei \& Alkhalifah, 2018). Depending on the illumination conditions, the number of parameters that are above a certain singular value threshold can vary. In the case of $\mathrm{P}$ waves, we previously presented the number of invertible parameters depending on the acquisition limitations (Kazei \& Alkhalifah, 2018). The optimal number of parameters for other types of scattering is a topic for future research.

(C)2019 American Geophysical Union. All Rights Reserved. 
In summary, this atlas describes the sensitivity of first-order scattered data to different scales of the parameters involved in describing arbitrary elastic anisotropy. It offers insight into what we can expect from the inversion of data considering the available components, i.e., azimuths and aperture. We can only invert for those medium perturbations to which the data are sensitive. Such medium perturbations could correspond to one or many anisotropic parameters; more importantly, they may include many scales. Thus, the map of medium-parameter perturbation sensitivity, as a function of model wavenumber, exposes the resolution space of the parameters. By combining the maps, we expose the potential tradeoffs at different scales and azimuths. In the maze of interpreting such multiparameter inversion results, resolution and tradeoff insights are crucial.

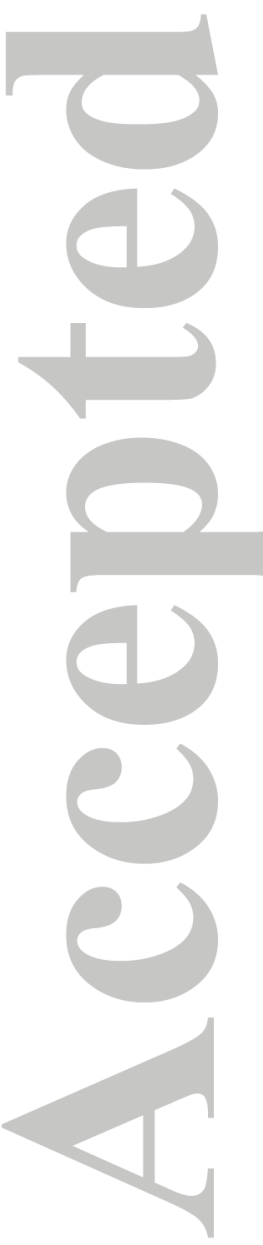

(C)2019 American Geophysical Union. All Rights Reserved. 


\section{Appendix A: Analytic properties of scattering radiation patterns}

In this section, we discuss the analytic properties of reflection-based patterns. These include reciprocity, reduction, symmetry, relation to the linearized reflection coefficient, and transmission patterns.

\section{A1. Reciprocity}

Symmetry of the elastic tensor $c_{i j k l}$ leads to the reciprocity for scattering functions:

$$
\begin{array}{r}
R_{\delta \mathbf{m}}(\mathbf{s}, \boldsymbol{\varsigma}, \mathbf{g}, \boldsymbol{\xi})=\boldsymbol{\varsigma} \cdot \boldsymbol{\xi} \delta \rho+\mathbf{s} \boldsymbol{\varsigma}: \delta \mathbf{c}: \mathbf{g} \boldsymbol{\xi}= \\
\boldsymbol{\xi} \cdot \boldsymbol{\varsigma} \delta \rho+\mathbf{g} \boldsymbol{\xi}: \delta \mathbf{c}: \mathbf{s} \boldsymbol{\varsigma}=R_{\delta \mathbf{m}}(\mathbf{g}, \boldsymbol{\xi}, \mathbf{s}, \boldsymbol{\varsigma})
\end{array}
$$

and diffraction-based radiation patterns:

$$
\mathcal{D}_{W I-W S, \delta \mathbf{m}}(\mathbf{s}, \mathbf{g})=\mathcal{D}_{W S-W I, \delta \mathbf{m}}(\mathbf{g}, \mathbf{s})
$$

This property is obvious, yet it can sometimes help to debug radiation patterns.

\section{A2. Reduction for isotropic parameters}

For isotropic parameters $(\lambda, \mu, \rho)$, the diffraction-based radiation patterns depend only on the opening scattering angle, defined by the scalar product $\mathbf{s} \cdot \mathbf{g}$ (R.-S. Wu \& Aki, 1985):

$$
\mathcal{D}_{i s o, W I-W S}(\mathbf{s}, \mathbf{g})=\mathcal{D}_{i s o, W I-W S}(\mathbf{s} \cdot \mathbf{g}) .
$$

Therefore, they are easily represented graphically as functions of a single scalar.

\section{A3. Reduction for TI parameters}

For transversely isotropic parameters, diffraction-based radiation patterns are invariant w.r.t. rotation around the symmetry axis $\mathbf{e}^{T I}$. Thus, the number of angles that define (C)2019 American Geophysical Union. All Rights Reserved. 
scattering can be reduced to three (Calvet et al., 2006):

$$
\mathcal{D}_{T I, W I-W S}(\mathbf{s}, \mathbf{g})=\mathcal{D}_{T I, W I-W S}\left(\mathbf{s} \cdot \mathbf{g}, \mathbf{s} \cdot \mathbf{e}^{T I}, \mathbf{g} \cdot \mathbf{e}^{T I}\right)
$$

where $T I$ denotes any parameter of the tilted transversely isotropic medium, and $\mathbf{s} \cdot \mathbf{e}^{T I}$ and

$\mathbf{g} \cdot \mathbf{e}^{T I}$ give the incident and scattered angles, respectively, measured from the symmetry axis $\mathbf{e}^{T I}$ of the background medium.

D.

\section{A4. Symmetry}

A radiation pattern is antisymmetric w.r.t. $\mathbf{s}$ for density $\rho$, i.e.,

$\mathcal{D}_{\rho, W I-W S}(-\mathbf{s}, \mathbf{g})=-\mathcal{D}_{\rho, W I-W S, \delta \mathbf{m}}(\mathbf{s} \cdot \mathbf{g})$

and symmetric for all elastic constants $c_{i j k l}$ :

$\mathcal{D}_{\mathbf{c}, W I-W S}(-\mathbf{s}, \mathbf{g})=\mathcal{D}_{\mathbf{c}, W I-W S, \delta \mathbf{m}}(\mathbf{s} \cdot \mathbf{g})$.

The consequence of (15) is that the density in full diffraction-based radiation patterns can always be distinguished from scattering by other parameters. Due to reciprocity, the same symmetry properties hold for the vector $\mathbf{g}$. In other than $C_{i j}, \rho$ parameterizations which include density (Tsvankin, 1997 ; Stovas, 2015 ; J.-W. Oh \& Alkhalifah, 2016), the symmetry properties (A6) may not apply.

\section{A5. Relation to linearized reflection coefficients}

Reflection-based radiation patterns are proportional to the linearized reflection coefficients (R. K. Shaw \& Sen, 2004), even though patterns are derived from Born approximation and reflection coefficients are derived from boundary conditions at a solid-solid interface. However, unlike the reflection-based radiation patterns, the coefficients depend on the scattering type and not the perturbed parameters.

(C)2019 American Geophysical Union. All Rights Reserved. 


\section{A6. Transmission-reflection pattern relation}

For monotypic $(\mathrm{WI}=\mathrm{WS})$ waves, the so-called transmission radiation patterns can be defined as

$\mathcal{T}_{W I, \delta \mathbf{m}}(\mathbf{g}) \equiv \mathcal{D}_{W I-W I, \delta \mathbf{m}}(-\mathbf{g}, \mathbf{g})=\frac{\partial c_{i j k l}}{\partial c_{p q r s}} g_{i} \xi_{j} g_{k} \xi_{l}$.

One interesting property of the transmission-based radiation patterns in the $C_{i j}, \rho$ parameterization is that none of them differ in shape from the corresponding reflection-based radiation patterns, for all monoclinic elastic parameters except density. The proof of this relation we provide below.

We first rewrite the reflection-based radiation pattern expression:

$$
\mathcal{R}_{c_{p q r s}, W I-W I}(\mathbf{g}) \equiv \frac{\partial c_{i j k l}}{\partial c_{p q r s}}(-1)^{1+\delta_{i 3}} g_{i}(-1)^{1+\delta_{j 3}} \xi_{j} g_{k} \xi_{l}=(-1)^{N(i, j)} \frac{\partial c_{i j k l}}{\partial c_{p q r s}} g_{i} \xi_{j} g_{k} \xi_{l}
$$

where we introduce a function

$$
N(p, q) \equiv \delta_{p 3}+\delta_{q 3}
$$

that counts the number of elements equal to three in a sequence of numbers.

For monoclinic parameters $c_{p q r s} N(p, q)$ and $N(r, s)$ are either odd or even together

$$
N(p, q) \equiv N(r, s)(\bmod 2)
$$

which is required by the symmetry of elastic equations w.r.t. the change in the direction of the third axis.

If $N(p, q)$ is even (either equal two or zero)

$$
N(p, q) \equiv N(r, s) \equiv 0(\bmod 2)
$$

(C)2019 American Geophysical Union. All Rights Reserved. 
i.e., for scattering on the orthorhombic parameters $\mathbf{C}_{i j}$, where $i \leq 3$ and $j \leq 3$, and $\mathbf{C}_{66}$, and the monoclinic parameters $\mathbf{C}_{16}, \mathbf{C}_{26}$, and $\mathbf{C}_{36}$ (in Voigt notation), then

$$
\mathcal{R}_{c_{p q r s}, W I-W I}(\mathbf{g}) \equiv \frac{\partial c_{i j k l}}{\partial c_{p q r s}} g_{i} \xi_{j} g_{k} \xi_{l}=\mathcal{T}_{c_{p q r s}, W I}(\mathbf{g})
$$

On the other hand, if $N(p, q) \bmod 2=N(r, s) \bmod 2=1$, i.e., for scattering on $\mathbf{C}_{44}, \mathbf{C}_{55}$, and the monoclinic parameter $\mathbf{C}_{45}$, then

$\mathcal{R}_{c_{p q r s}, W I-W I}(\mathbf{g}) \equiv-\frac{\partial c_{i j k l}}{\partial c_{p q r s}} g_{i} \xi_{j} g_{k} \xi_{l}=-\mathcal{T}_{c_{p q r s}, W I}(\mathbf{g})$

\section{A7. Monoclinic - monotypic}

For non-monoclinic parameters $c_{p q r s}$,

$N(p, q) \neq N(r, s) \bmod 2$.

Without any loss of generality we assume that $N(p, q) \bmod 2=1$ and in particular $p=3$. Automatically $q \neq p$ and therefore:

$$
\begin{array}{r}
\mathcal{R}_{c_{3 q r s}, W I-W I}(\mathbf{g}) \equiv(-1)^{\delta_{i 3}+\delta_{j 3}} \frac{\partial c_{i j k l}}{\partial c_{3 q r s}} g_{i} \xi_{j} g_{k} \xi_{l}= \\
-\frac{\partial c_{3 q k l}}{\partial c_{3 q r s}} g_{p} \xi_{q} g_{k} \xi_{l}-\frac{\partial c_{q 3 k l}}{\partial c_{3 q r s}} g_{q} \xi_{p} g_{k} \xi_{l}+ \\
\frac{\partial c_{k l 3 q}}{\partial c_{3 q r s}} g_{p} \xi_{q} g_{k} \xi_{l}+\frac{\partial c_{k l q 3}}{\partial c_{3 q r s}} g_{q} \xi_{p} g_{k} \xi_{l} \equiv 0
\end{array}
$$

We link the vertical resolution directly to the reflection-based radiation patterns in the next section, which finalizes our review of the radiation-pattern properties.

\section{Appendix B: Born approximation validity}

The Born approximation for scattering (equation (9)) is valid for relatively small

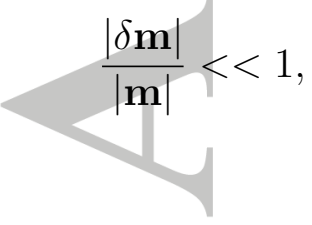

(C)2019 American Geophysical Union. All Rights Reserved. 
and local

$$
\frac{\omega d}{V_{0}} \frac{|\delta \mathbf{m}|}{|\mathbf{m}|}<<1
$$

perturbations $\delta \mathbf{m}$, where $V_{0}$ is the smallest background velocity ( $V_{s}$ in the isotropic case), and $d$ is the diameter of the perturbation. The above assumptions are best met in the case of low-contrast point scatterers. For instance, the approximation is valid for perturbations in thin, low-contrast layers and near-normal wave incidence angles (R. K. Shaw \& Sen, 2004).

The Born approximation does not imply specific shape restrictions on the scattering perturbation or the background medium. Therefore, modern methods for the dynamic inversion of seismic wavefields, including linearized reverse-time migration (LSRTM) and every iteration of full-waveform inversion (FWI), rely heavily on it. Thus, we can analyze the resolution of these methods by exploring the sensitivity kernels in the spatial Fourier domain (Devaney, 1984 ; R. Wu \& Toksöz, 1987 ; Mora, 1989 ; Sirgue \& Pratt, 2004 ; Kazei, Troyan, et al., 2013 ; Kazei, Kashtan, et al., 2013 ; Podgornova et al., 2015 ; Kazei \& Alkhalifah, 2017).

\section{Appendix C: Non-scattering combinations \\ C1. Non-scattering combinations for SH waves}

Namely, by writing down Snell's law for propagation directions,

$$
\begin{aligned}
& s_{1}=-g_{1}=\cos \theta \cos \varphi \\
& s_{2}=-g_{2}=\cos \theta \sin \varphi
\end{aligned}
$$

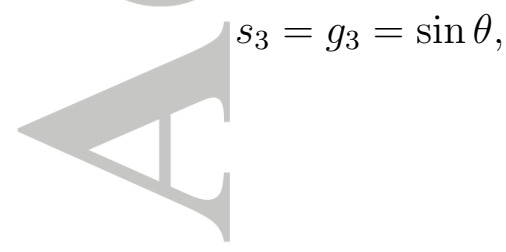

(C)2019 American Geophysical Union. All Rights Reserved. 
and $S H$ wave polarization,

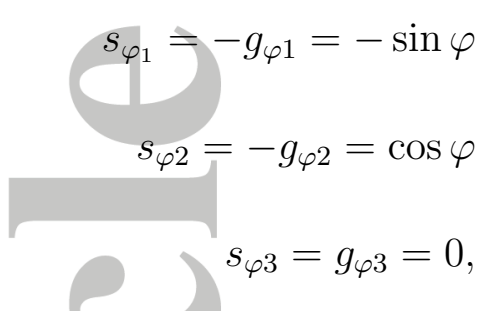

we can easily obtain the radiation pattern for density as

$\mathcal{R}_{S H-S H, \rho}=\mathbf{s}_{\varphi} \cdot \mathbf{g}_{\varphi}=-1$

Then, with a little more effort, we find the reflection-based radiation patterns for azimuthally independent scattering combinations of elastic constants as

$$
\begin{aligned}
& \mathcal{R}_{\mathbf{C}_{44}, S H-S H}=\mathcal{R}_{\mathbf{c}_{3232}, S H-S H}=-\left(s_{3} s_{\varphi 2}\right)^{2}=-\sin ^{2} \theta \cos ^{2} \varphi \\
& \mathcal{R}_{\mathbf{C}_{55}, S H-S H}=\mathcal{R}_{\mathbf{c}_{3131}, S H-S H}=-\left(s_{3} s_{\varphi 1}\right)^{2}=-\sin ^{2} \theta \sin ^{2} \varphi .
\end{aligned}
$$

Therefore,

$$
\mathcal{R}_{\mathbf{C}_{44}+\mathbf{C}_{55}, S H-S H}=-\sin ^{2} \theta .
$$

Analogously,

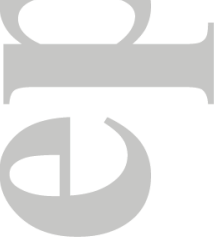

$$
\begin{array}{r}
\mathcal{R}_{\mathbf{C}_{12}, S H-S H}=\mathcal{R}_{\mathbf{c}_{1122}, S H-S H}=2\left(s_{1} s_{\varphi 1}\right)\left(s_{2} s_{\varphi 2}\right)= \\
-2 \cos ^{2} \theta \cos ^{2} \varphi \sin ^{2} \varphi=-\frac{1}{2} \cos ^{2} \theta \sin ^{2} 2 \varphi
\end{array}
$$

$\mathcal{R}_{\mathbf{C}_{66}, S H-S H}=\mathcal{R}_{\mathbf{c}_{1212}, S H-S H}=\left(s_{1} s_{\varphi 2}\right)^{2}+\left(s_{2} s_{\varphi 1}\right)^{2}+2 s_{1} s_{2} s_{\varphi_{1}} s_{\varphi_{2}}=$

$$
\cos ^{2} \theta\left(\cos ^{2} \varphi-\sin ^{2} \varphi\right)^{2}=\cos ^{2} \theta \cos ^{2} 2 \varphi
$$

and, hence,

$\mathcal{R}_{\mathbf{C}_{12}+\mathbf{C}_{66}, S H-S H}=\cos ^{2}(\bigoplus 2019$ American Geophysical Union. All Rights Rese(ryed). 
Finally, we can construct combination that scatters just like density:

$\mathcal{R}_{\mathbf{C}_{44}+\mathbf{C}_{55}-\mathbf{C}_{12}-\mathbf{C}_{66}, S H-S H}=-1$

\section{C2. SV-SV waves}

We combine Snell's law for propagation directions $(\mathrm{C} 0)$ and its form for $S V$ polarization

$$
\begin{aligned}
& \text { directions } \mathbf{s}_{\theta} \text { and } \mathbf{g}_{\theta}, \\
& s_{\theta 1}=-g_{\theta 1}=-\sin \theta \cos \varphi \\
& s_{\theta 2}=-g_{\theta 2}=-\sin \theta \sin \varphi \\
& s_{\theta 3}=g_{\theta 3}=\cos \theta,
\end{aligned}
$$

with the partial derivative $\frac{\partial \mathbf{C}}{\partial \mathbf{m}}$ expression for the new parameterization (35) to derive the reflection-based radiation patterns for $\varepsilon_{d}$ :

$$
\begin{aligned}
& \mathcal{R}_{S V-S V, \varepsilon_{d}}=\mathcal{R}_{S V-S V, 2 \mathbf{C}_{22}+\mathbf{C}_{23}}=2\left(s_{2} s_{\theta_{2}}\right)^{2}+2\left(s_{2} s_{\theta_{2}}\right)\left(g_{3} g_{\theta_{3}}\right)= \\
& 2(\cos \theta \sin \varphi \sin \theta \sin \varphi)^{2}-2(\cos \theta \sin \varphi \sin \theta \sin \varphi \sin \theta \cos \theta)= \\
& \frac{1}{2}\left(\sin ^{2} 2 \theta \sin ^{4} \varphi-\sin ^{2} 2 \theta \sin ^{2} \varphi\right)= \\
& \frac{1}{2} \sin ^{2} 2 \theta \sin ^{2} \varphi\left(\sin ^{2} \varphi-1\right)= \\
& -\frac{1}{8} \sin ^{2} 2 \theta \sin ^{2} 2 \varphi \\
& 2 \cos ^{2} \theta \sin ^{2} \theta \cos ^{2} \varphi \sin ^{2} \varphi= \\
& \frac{1}{8} \sin ^{2} 2 \theta \sin ^{2} 2 \varphi \text {. }
\end{aligned}
$$




\section{Appendix D: Snell's law in wavenumber domain}

For $S-S$ scattering, the vectors pointing towards the source and the receiver are easily

found for a given $k_{z} \in[0,2]$ :

$s_{1}=-g_{1}=\frac{1}{V_{s}} \sqrt{1-\frac{k_{z}^{2}}{4}} \cos \varphi, s_{2}=-g_{2}=\frac{1}{V_{s}} \sqrt{1-\frac{k_{z}^{2}}{4}} \sin \varphi, s_{3}=g_{3}=\frac{1}{V_{s}} k_{z} / 2$.

For $P-P$ scattering on a horizontal reflector, the vectors pointing towards the source and the receiver are easily found for a given $k_{z} \in[0,2 \varkappa]$ :

$s_{1}=-g_{1}=\frac{1}{V_{p}} \sqrt{1-\frac{k_{z}^{2}}{4 \varkappa^{2}}} \cos \varphi, s_{2}=-g_{2}=\frac{1}{V_{p}} \sqrt{1-\frac{k_{z}^{2}}{4 \varkappa^{2}}} \sin \varphi, s_{3}=g_{3}=\frac{1}{V_{p}} \frac{k_{z}}{2 \varkappa}$

For $P-S$ scattering, Snell's law:

$\mathbf{s}+\mathbf{g}=\mathbf{K}_{z}$

leads to the following expressions:

$s_{x y}=\frac{1}{V_{s}} \sqrt{2 \varkappa^{2} k_{z}^{2}-\varkappa^{4}+2 \varkappa^{2}-k_{z}^{4}+2 k_{z}^{2}-1} /\left(2 k_{z}\right)$,
$s_{1}=-g_{1}=s_{x y} \cos (\varphi), s_{2}=-g_{2}=s_{x y} \sin (\varphi)$,
$s_{3}=\frac{1}{V_{p}}\left(\varkappa^{2}+k_{z}^{2}-1\right) /\left(2 \varkappa k_{z}\right), g_{3}=\frac{1}{V_{s}}\left(k_{z}^{2}-\varkappa^{2}+1\right) /\left(2 k_{z}\right)$,

where $\varkappa=\frac{V_{s}}{V_{p}}$ is the ratio of the shear and longitudinal velocities. We show all the possible reflection-based radiation patterns for other cases mapped from the $\varphi, \theta$ domain into the wavenumber domain $\left(\varphi, k_{z}\right)$ as spectral sensitivities in order to directly access the resolution of inversion the normalized wavenumber domain.

\section{Appendix E: Conclusions}

We remapped reflection-based radiation patterns into the spectral domain for all the anisotopic elastic parameters, and incident and scattered wave types. Each remapped pat-

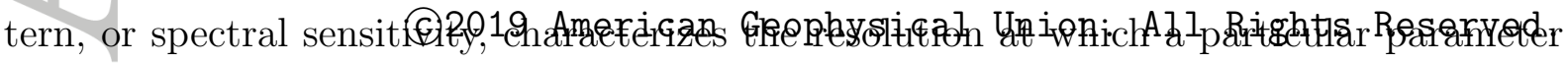


can be reconstructed. We found some apparent tradeoffs among the triclinic parameters, and showed that only monoclinic parameters can be robustly reconstructed from monotypic waves. Table 1 summarizes the tradeoffs found for monotypic waves.

For the orthorhombic parameters, we performed SVD and found the exact null-spaces for each mode of scattering. The simple intersections of the null-spaces provide information about the null-spaces themselves when several modes are used in the inversion. The intersections of $P-P$ waves, which are used together with $P-S$ conversion, and the tradeoffs among the converted waves are provided in Table 2 . From the $P-P$ and $P-S V$ waves, we can reconstruct all the parameters except $\gamma_{1}$; from $P-S H$ waves, we can theoretically reconstruct all the orthorhombic parameters.

In realistic scenarios, limited apertures, frequency content, and noise in the data may limit the set of recoverable parameters, while prior assumptions introduced via regularization or constraints can expand the set of recoverable parameters. This paper will help researchers choose the optimal constraints, regularizations, and parameterizations when designing elastic anisotropic inversions.

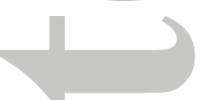

\section{Appendix F: Acknowledgments}

The research reported in this publication was supported by funding from King Abdullah University of Science and Technology (KAUST). We are grateful to seismic wave analysis group, KAUST, for helpful discussions. Vladimir Kazei is also grateful to Oleg Ovcharenko and Veronica Tremblay of KAUST for their suggestions on improving the manuscript readability. We thank KAUST for support and its HPC resources. Finally, we would like to thank JGR editors and reviewers for their helpful suggestions and comments. The MATLAB code used in the paper is available at https://github.com/vkazei/ScatteringAtlas.

(C)2019 American Geophysical Union. All Rights Reserved. 


\section{Glossary}

$\omega$ - angular frequency;

$\mathbf{x}$ - imaging point vector;

$\mathbf{x}_{s}, \mathbf{x}_{g}-$ source and geophone radius vectors, respectively;

$\mathbf{s}$ - unit vector pointing towards the source along the source wavepath direction;

$\mathbf{g}$ - same as $\mathbf{s}$ but towards the geophone;

$\varsigma, \xi \rightarrow$ polarization vectors for the incident and scattered waves respectively

$G_{i k}\left(\mathbf{x}, \mathbf{x}^{\prime}\right) \equiv G_{i k}\left(\mathbf{x}, \mathbf{x}^{\prime}, \omega\right)$ - Green's tensor in the background medium for frequency $\omega ;$

$\mathbf{K}$ - wavenumber domain vector;

$\mathbf{u}\left(\mathbf{x}_{s}, \mathbf{x}_{g}\right)$ - monochromatic displacement wavefield at frequency $\omega ;$

$c_{i j k l}, \mathbf{c}-$ stiffness tensor;

$C_{i j}, \mathbf{C}-$ stiffness matrix in Voigt notation;

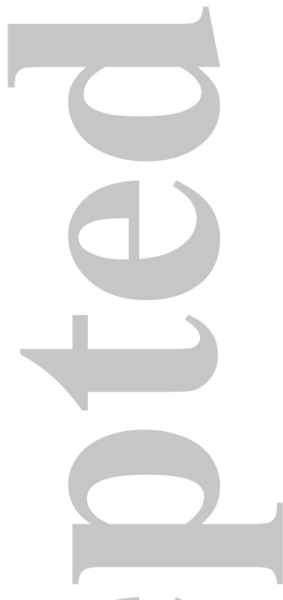

$\longrightarrow$

(C)2019 American Geophysical Union. All Rights Reserved. 


\section{Hierarchical parameters from elastic stiffnesses}

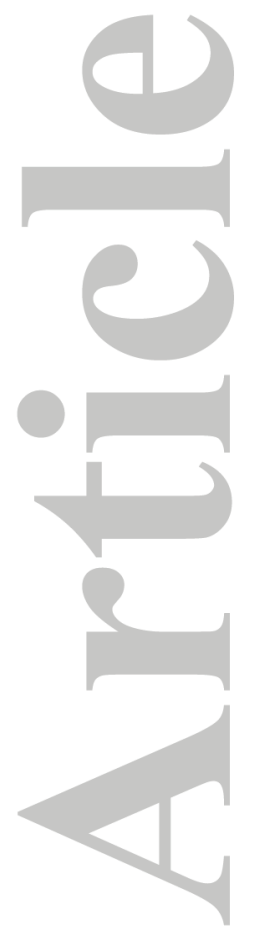

$$
\begin{aligned}
& C_{11}=\rho V_{p}^{2}, \\
& C_{22}=\left(1+2 \varepsilon_{d}\right) \rho V_{p}^{2}, \\
& C_{33}=\frac{1}{1+2 \varepsilon_{1}} \rho V_{p}^{2}, \\
& C_{12}=\rho \sqrt{\left(V_{p}^{2}-\left(1+2 \gamma_{1}\right) V_{s}^{2}\right)} \\
& \times \sqrt{\left(\left(1+2 \delta_{3}\right) V_{p}^{2}-\left(1+2 \gamma_{1}\right) V_{s}^{2}\right)} \\
& -\left(1+2 \gamma_{1}\right) \rho V_{s}^{2}, \\
& C_{13}=\rho \sqrt{\left(\frac{V_{p}^{2}}{1+2 \varepsilon_{1}}-V_{s}^{2}\right)\left(\frac{V_{p}^{2}}{1+2 \eta_{1}}-V_{s}^{2}\right)} \\
& -\rho V_{s}^{2} \\
& C_{23}=\rho\left[\left(\frac{V_{p}^{2}}{1+2 \varepsilon_{1}}\left(1+2 \gamma_{d}\right) V_{s}^{2}\right)\right. \\
& \left.\times\left(\frac{V_{p}^{2}\left(1+\varepsilon_{d}\right)}{\left(1+2 \eta_{1}\right)\left(1+2 \eta_{d}\right)}-\left(1+2 \gamma_{d}\right) V_{s}^{2}\right)\right]^{1 / 2} \\
& -\left(1+2 \gamma_{d}\right) \rho V_{s}^{2}, \\
& C_{44}=\left(1+2 \gamma_{d}\right) \rho V_{s}^{2}, \\
& C_{55}=\rho V_{s}^{2}, \\
& C_{66}=\left(1+2 \gamma_{1}\right) \rho V_{s}^{2} \text {. }
\end{aligned}
$$

$\rho, \lambda-$ density and first lam parameter;

$\delta f$ - variational perturbation of a function $f$;

$\hat{f}-$ Fourier transform of a function $f$;

$W I-W S, \delta \mathbf{m}-$ incident and scattered wave types and the perturbation parameter type (C)2019 American Geophysical Union. All Rights Reserved. 
$W I-W S, \delta \mathbf{m}=\mathrm{P}-\mathrm{P}, \lambda$ or $W I-W S, \delta \mathbf{m}=\mathrm{P}-\mathrm{SH}, C_{13}$

$\mathcal{R}_{W I-W S, \delta \mathbf{m}}(\mathbf{s}, \mathbf{g})$ - radiation pattern for plane waves and parameter perturbation of type $W I-W S, \delta \mathbf{m}$

$(\mathbf{s g})_{i j} \equiv s_{i} g_{j}-$ the outer (dyadic) product of tensors (vectors);

:- convolution of tensors on two indexes, e.g., $\mathbf{c}: \mathbf{s g} \equiv c_{i j k l} s_{k} g_{l}$;

Einstein summation is assumed throughout the paper.

We choose the Fourier convention following (Hudson \& Heritage, 1981):

$u(t)=\int_{-\infty}^{+\infty} \exp (-i \omega t) U(\omega) \mathrm{d} \omega, \hat{U}(\mathbf{K})=\int_{V} \exp (-i \mathbf{K} \mathbf{x}) U(\mathbf{x}) \mathrm{d} \mathbf{x}$.

Natural units convention and equivalent normalization:

Scattering should depend only on the geometric properties of the elasticity tensor; therefore, we can normalize elastic parameters. The latter is equivalent to the following assumptions: $V_{p}=1, \rho=1$ about the background isotropic medium, similar to the natural units convention. Slownesses are made dimensionless by multiplying by $V_{0 p}$ :

$\overline{\mathbf{s}}=V_{0 p} * \mathbf{s}$,

densities are normalized by $\rho_{0}$

$\bar{\rho}=\frac{\rho}{\rho_{0}}$

therefore elastic constants $C_{i j}$ and components of elastic tensor $c_{i j k l}$ are normalized by $\rho_{0} V_{0 p}^{2} . V_{0 p}$ is the $P$ wave velocity in the background media throughout the paper.

\section{Références}

Alkhalifah, T. (2000). An acoustic wave equation for anisotropic media. Geophysics, $65(4), 1239-1250$.

(C)2019 American Geophysical Union. All Rights Reserved. 
Alkhalifah, T. (2015). Scattering-angle based filtering of the waveform inversion gradients. Geophysical Journal International, 200(1), 363-373. 10.1093/gji/ggu379

Alkhalifah, T. (2016, avril). Full-model wavenumber inversion: An emphasis on the appropriate wavenumber continuation. GEOPHYSICS, 81(3), R89-R98. 10.1190/geo2015-0537.1

Alkhalifah, T., \& Plessix, R.-E. (2014). A recipe for practical full-waveform inversion in anisotropic media: An analytical parameter resolution study. Geophysics, 79 (3), R91-R101. 10.1190/geo2013-0366.1

Anikiev, D., Kazei, V., Kashtan, B., Ponomarenko, A., Troyan, V., \& Shigapov, R. (2014). Methods of seismic waveform inversion. Seismic Technology, 11(1), 1-32.

Babuska, V., \& Cara, M. (1991). Seismic Anisotropy in the Earth. Springer Science \& Business Media.

Beretta, M., Bernasconi, G., \& Drufuca, G. (2002, janvier). AVO and AVA inversion for fractured reservoir characterization. GEOPHYSICS, 67(1), 300-306.

$10.1190 / 1.1451802$

Beylkin, G., \& Burridge, R. (1990). Linearized inverse scattering problems in acoustics and elasticity. Wave motion, 12(1), 15-52.

Bolt, B. A. (1970, septembre). PdP and PKiKP Waves and Diffracted PcP Waves. Geophysical Journal of the Royal Astronomical Society, 20(4), 367-382. 10.1111/j.1365246X.1970.tb06080.x

Bóna, A., Bucataru, I., \& Slawinski, M. A. (2007, juin). Coordinate-free Characterization of the Symmetry Classes of Elasticity Tensors. Journal of Elasticity, 87(2-3), 109132. $10.1007 / \mathrm{s} 10659-007-9099-\mathrm{Z}$

(C)2019 American Geophysical Union. All Rights Reserved. 
Bozdăg, E., Peter, D., Lefebvre, M., Komatitsch, D., Tromp, J., Hill, J.Pugmire, D. (2016). Global adjoint tomography: First-generation model. Geophysical Supplements to the Monthly Notices of the Royal Astronomical Society, 207(3), 1739-1766.

Brossier, R., Operto, S., \& Virieux, J. (2009, novembre). Seismic imaging of complex onshore structures by $2 \mathrm{D}$ elastic frequency-domain full-waveform inversion. GEOPHYSICS, 74(6), WCC105-WCC118. 10.1190/1.3215771

Browaeys, J. T., \& Chevrot, S. (2004, novembre). Decomposition of the elastic tensor and geophysical applications. Geophysical Journal International, 159(2), 667-678. 10.1111/j.1365-246X.2004.02415.x

Calvet, M., Chevrot, S., \& Souriau, A. (2006). P-wave propagation in transversely isotropic media: I. Finite-frequency theory. Physics of the Earth and Planetary Interiors, 156(1-2), 12-20. http://dx.doi.org/10.1016/j.pepi.2006.01.004

Cheverda, V. A., \& Kostin, V. I. (1995). R-pseudoinverses for compact operators in Hilbert spaces: Existence and stability. Journal of Inverse and Ill-Posed Problems, $3(2), 131-148$.

Creager, K. C. (1999). Large-scale variations in inner core anisotropy. Journal of Geophysical Research: Solid Earth, 104(B10), 23127-23139.

De Hoop, M. V., Spencer, C., \& Burridge, R. (1999). The resolving power of seismic amplitude data: An anisotropic inversion/migration approach. Geophysics, 64(3), $852-873$

den Boer, L. D., \& Sayers, C. M. (2018, janvier). Constructing a discrete fracture network constrained by seismic inversion data. Geophysical Prospecting, 66(1), 124140. $10.1111 / 1365-2478.12527$

(C)2019 American Geophysical Union. All Rights Reserved. 
Devaney, A. J. (1984, janvier). Geophysical Diffraction Tomography. IEEE Transactions on Geoscience and Remote Sensing, GE-22(1), 3-13. 10.1109/TGRS.1984.350573

Dokter, E., Köhn, D., Wilken, D., De Nil, D., \& Rabbel, W. (2017, décembre). Full waveform inversion of SH- and Love-wave data in near-surface prospecting. Geophysical Prospecting, 65(S1), 216-236. 10.1111/1365-2478.12549

Durham, W. B., \& Goetze, C. (1977, décembre). Plastic flow of oriented single crystals of olivine: 1. Mechanical data. Journal of Geophysical Research, 82(36), 5737-5753. 10.1029/JB082i036p05737

Eaton, D. W. S., \& Stewart, R. R. (1994). Migration/inversion for transversely isotropic elastic media. Geophysical Journal International, 119(2), 667-683. 10.1111/j.1365246X.1994.tb00148.x

Esser, E., Guasch, L., van Leeuwen, T., Aravkin, A. Y., \& Herrmann, F. J. (2016, août). Total-variation regularization strategies in full-waveform inversion. arxiv.org. Consulté le 2018-12-14, sur https://arxiv.org/abs/1608.06159

Ewald, P. (1969). Introduction to the dynamical theory of X-ray diffraction. Acta Crystallographica Section A: Crystal Physics, Diffraction, Theoretical and General Crystallography, 25(1), 103-108.

Fukao, Y. (1984, juin). Evidence from core-reflected shear waves for anisotropy in the Earth's mantle. Nature, $309(5970), 695-698$. 10.1038/309695a0

Gholami, Y., Brossier, R., Operto, S., Ribodetti, A., \& Virieux, J. (2013a). Which parameterization is suitable for acoustic vertical transverse isotropic full waveform inversion? Part 1: Sensitivity and trade-off analysis. GEOPHYSICS, 78(2), R81R105. 10.1190/geo2012-0204.1

(C)2019 American Geophysical Union. All Rights Reserved. 
Gholami, Y., Brossier, R., Operto, S., Ribodetti, A., \& Virieux, J. (2013b, mars). Which parameterization is suitable for acoustic vertical transverse isotropic full waveform inversion? Part 1: Sensitivity and trade-off analysis. GEOPHYSICS, 78(2), R81R105. 10.1190/geo2012-0204.1

Grechka, V., Contreras, P., \& Tsvankin, I. (2000, mai). Inversion of normal moveout for monoclinic media1. Geophysical Prospecting, 48(3), 577-602. 10.1046/j.13652478.2000.00200.x

He, W., \& Plessix, R.-E. (2016). Analysis of different parameterisations of waveform inversion of compressional body waves in an elastic transverse isotropic Earth with a vertical axis of symmetry. Geophysical Prospecting, n/a-n/a. 10.1111/13652478.12452

Hudson, J., \& Heritage, J. (1981). The use of the Born approximation in seismic scattering problems. Geophysical Journal International, 66(1), 221-240.

Irving, J. C. E., \& Deuss, A. (2015, décembre). Regional seismic variations in the inner core under the North Pacific. Geophysical Journal International, 203(3), 2189-2199. $10.1093 /$ gji/ggv435

Ivanov, Y., \& Stovas, A. (2016). Upscaling in orthorhombic media: Behavior of elastic parameters in heterogeneous fractured earth. GEOPHYSICS, 81(3), C113-C126. 10.1190/geo2015-0392.1

Kalita, M., Kazei, V., Choi, Y., \& Alkhalifah, T. (2018, août). Regularized full-waveform inversion for salt bodies. In SEG Technical Program Expanded Abstracts 2018 (p. 1043-1047). Society of Exploration Geophysicists. Consulté le 2018-12-14, sur https://library.seg.org/doi/abs/10.1190/segam2018 (C)2019 American Geophysical Union. All Rights Reserved. 
-2995963.1 10.1190/segam2018-2995963.1

Kamath, N., \& Tsvankin, I. (2016). Elastic full-waveform inversion for VTI media: Methodology and sensitivity analysis. GEOPHYSICS, 81(2), C53-C68. $10.1190 /$ geo2014-0586.1

Kazei, V., \& Alkhalifah, T. (2017). On the Resolution of Inversion for Orthorhombic Anisotropy. In 79th EAGE Conference and Exhibition $201 \%$.

Kazei, V., \& Alkhalifah, T. (2018). Waveform inversion for orthorhombic anisotropy with P-waves: Feasibility \&amp; resolution. Geophysical Journal International, ggy034. $10.1093 /$ gji/ggy034

Kazei, V., Kalita, M., \& Alkhalifah, T. (2017, juin). Salt-body Inversion with Minimum Gradient Support and Sobolev Space Norm Regularizations. In 79th EAGE Conference and Exhibition 2017. 10.3997/2214-4609.201700600

Kazei, V., Kashtan, B., Troyan, V., \& Mulder, W. (2015). FWI spectral sensitivity analysis in the presence of a free surface. In SEG Technical Program Expanded Abstracts 2015 (p. 1415-1419). Society of Exploration Geophysicists.

Kazei, V., Kashtan, B. M., Troyan, V. N., \& Mulder, W. A. (2013). Spectral Sensitivity Analysis of FWI in a Constant-gradient Background Velocity Model. In 75th EAGE Conference 8 Exhibition incorporating SPE EUROPEC 2013. 10.3997/22144609.20130599

Kazei, V., Tessmer, E., \& Alkhalifah, T. (2016). Scattering angle-based filtering via extension in velocity. In SEG Technical Program Expanded Abstracts 2016 (p. 11571162). Society of Exploration Geophysicists.

Kazei, V., Troyan, V., Kashtan, B., \& Mulder, W. (2013). On the role of reflections, (C)2019 American Geophysical Union. All Rights Reserved. 
refractions and diving waves in full-waveform inversion. Geophysical Prospecting, $61(6), 1252-1263$.

Köhn, D., De Nil, D., Kurzmann, A., Przebindowska, A., \& Bohlen, T. (2012, octobre). On the influence of model parametrization in elastic full waveform tomography. Geophysical Journal International, 191(1), 325-345. 10.1111/j.1365-246X.2012.05633.x

Köhn, D., Hellwig, O., De Nil, D., \& Rabbel, W. (2015). Waveform inversion in triclinic anisotropic media - a resolution study. Geophysical Journal International, 201(3), $1642-1656$.

Kurzmann, A., Gaßner, L., Thiel, N., Kunert, M., Shigapov, R., Wittkamp, F.Metz, T. (2016). Seismic Applications of Full Waveform Inversion. In W. E. Nagel, D. H. Kröner, \& M. M. Resch (Eds.), High Performance Computing in Science and Engineering 16 (p. 647-665). Cham : Springer International Publishing.

Long, M. D., \& Becker, T. W. (2010). Mantle dynamics and seismic anisotropy. Earth and Planetary Science Letters, $297(3), 341-354$.

Masmoudi, N., \& Alkhalifah, T. (2018, juin). Full-waveform inversion in acoustic orthorhombic media and application to a North Sea data set. GEOPHYSICS, 83(5), C179-C193. 10.1190/geo2017-0738.1

Mora, P. (1989). Inversion = migration + tomography. Geophysics, 54 (12), 1575-1586. $10.1190 / 1.1442625$

Mulder, W. A., \& Plessix, R.-E. (2008). Exploring some issues in acoustic full waveform inversion. Geophysical Prospecting, 56(6), 827-841. 10.1111/j.13652478.2008.00708.x

Oh, J., \& Alkhalifah, T. (2018, mars). Optimal full waveform inversion strategy for (C)2019 American Geophysical Union. All Rights Reserved. 
marine data in azimuthally rotated elastic orthorhombic media. GEOPHYSICS, 1-61. 10.1190/geo2017-0762.1

Oh, J.-W., \& Alkhalifah, T. (2016). Elastic orthorhombic anisotropic parameter inversion: An analysis of parameterization. GEOPHYSICS, 81(6), C279-C293. 10.1190/geo2015-0656.1

Operto, S., Miniussi, A., Brossier, R., Combe, L., Métivier, L., Monteiller, V.Virieux, J. (2015, août). Efficient 3-D frequency-domain mono-parameter full-waveform inversion of ocean-bottom cable data: Application to Valhall in the visco-acoustic vertical transverse isotropic approximation. Geophysical Journal International, 202(2), 13621391. $10.1093 /$ gji/ggv226

Ovchärenko, O., Kazei, V., Peter, D., \& Alkhalifah, T. (2018). Variance-based salt body reconstruction for improved full-waveform inversion. Geophysics.

Pan, Y., Xia, J., Xu, Y., Gao, L., \& Xu, Z. (2015, novembre). Love-wave waveform inversion in time domain for shallow shear-wave velocity. GEOPHYSICS, 81(1), R1-R14. 10.1190/geo2014-0225.1

Peng, Z., Koper, K. D., Vidale, J. E., Leyton, F., \& Shearer, P. (2008, septembre). Inner-core fine-scale structure from scattered waves recorded by LASA. Journal of Geophysical Research: Solid Earth, 113(B9), B09312. 10.1029/2007JB005412

Podgornova, O., Leaney, S., \& Liang, L. (2018, mars). Resolution of VTI anisotropy with elastic full-waveform inversion: Theory and basic numerical examples. Geophysical Journal International, ggy116-ggy116. 10.1093/gji/ggy116

Podgornova, O., Leaney, S., Liang, L., \& others. (2015). Analysis of resolution limits of VTI anisotropy with full waveform inversion. In 2015 SEG Annual Meeting.

(C)2019 American Geophysical Union. All Rights Reserved. 
Prieux, V., Brossier, R., Operto, S., \& Virieux, J. (2013, septembre). Multiparameter full waveform inversion of multicomponent ocean-bottom-cable data from the Valhall field. Part 2: Imaging compressive-wave and shear-wave velocities. Geophysical Journal International, 194(3), 1665-1681. 10.1093/gji/ggt178

Raknes, E. B., \& Arntsen, B. (2014). Time-lapse full-waveform inversion of limited-offset seismic data using a local migration regularization. Geophysics, 79(3), WA117WA128.

Rüger, A. (1997). P-wave reflection coefficients for transversely isotropic models with vertical and horizontal axis of symmetry. Geophysics, 62(3), 713-722.

Schoenberg, M., \& Helbig, K. (1997, novembre). Orthorhombic media: Modeling elastic wave behavior in a vertically fractured earth. GEOPHYSICS, 62(6), 1954-1974. $10.1190 / 1.1444297$

Schuster, G. T., \& Huang, Y. (2014, décembre). Far-field superresolution by imaging of resonant multiples. Geophysical Journal International, 199(3), 1943-1949. $10.1093 /$ gji/ggu350

Shaw, R., \& Sen, M. (2006, mai). Use of AVOA data to estimate fluid indicator in a vertically fractured medium. GEOPHYSICS, 71 (3), C15-C24. 10.1190/1.2194896 Shaw, R. K., \& Sen, M. K. (2004). Born integral, stationary phase and linearized reflection coefficients in weak anisotropic media. Geophysical Journal International, $158(1), 225-238$.

Sieminski, A., Liu, Q., Trampert, J., \& Tromp, J. (2007, mars). Finite-frequency sensitivity of surface waves to anisotropy based upon adjoint methods. Geophysical Journal International, 168(3), 1153-1174. 10.1111/j.1365-246X.2006.03261.x

(C)2019 American Geophysical Union. All Rights Reserved. 
Silver, P. G., \& Chan, W. W. (1991, septembre). Shear wave splitting and subcontinental mantle deformation. Journal of Geophysical Research: Solid Earth, 96(B10), 1642916454. 10.1029/91JB00899

Sirgue, L., \& Pratt, R. (2004). Efficient waveform inversion and imaging: A strategy for selecting temporal frequencies. Geophysics, 69(1), 231-248. 10.1190/1.1649391

Snieder, R. (1986, mars). 3-D linearized scattering of surface waves and a formalism for surface wave holography. Geophysical Journal International, 84(3), 581-605. 10.1111/j.1365-246X.1986.tb04372.x

Snieder, R. (2002). Chapter 1.7.1 - General Theory of Elastic Wave Scattering. In R. Pike \& P. Sabatier (Eds.), Scattering (p. 528-542). London : Academic Press. Disponible sur http://www.sciencedirect.com/science/article/pii/ B9780126137606500279 http://dx.doi.org/10.1016/B978-012613760-6/50027-9

Stenhjem Hagen, V., \& Arntsen, B. (2017, avril). Parameter resolution and cross-talk for Elastic Full Wavefrom Inversion. In EGU General Assembly Conference Abstracts (Vol. 19, p. 7013). Consulté le 2018-03-13, sur http://adsabs.harvard.edu/abs/ 2017EGUGA . . 19.7013S

Stovas, A. (2015, novembre). Azimuthally dependent kinematic properties of orthorhombic media. GEOPHYSICS, 80(6), C107-C122. 10.1190/geo2015-0288.1

Stovas, A. (2017). Kinematic parameters of pure- and converted-mode waves in elastic orthorhombic media. Geophysical Prospecting, 65(2), 426-452.10.1111/13652478.12420

Tarantola, A. (1986). A strategy for nonlinear elastic inversion of seismic reflection data.

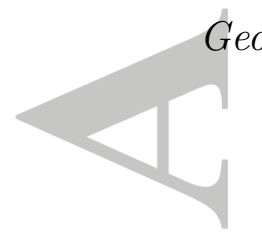
Geophysics, 51(10), 1893-1903.

(C)2019 American Geophysical Union. All Rights Reserved. 
Thomsen, L. (1986). Weak elastic anisotropy. GEOPHYSICS, 51(10), 1954-1966. $10.1190 / 1.1442051$

Tommasi, A., Knoll, M., Vauchez, A., Signorelli, J. W., Thoraval, C., \& Logé, R. (2009, juin). Structural reactivation in plate tectonics controlled by olivine crystal anisotropy. Nature Geoscience, 2(6), 423-427. 10.1038/ngeo528

Tromp, J. (1993). Support for anisotropy of the Earth's inner core from free oscillations. Nature, 366(6456), 678-681.

Tsvankin, I. (1997). Anisotropic parameters and P-wave velocity for orthorhombic media. Geophysics, 62(4), 1292-1309.

Vidale, J. E., \& Earle, P. S. (2000, mars). Fine-scale heterogeneity in the Earth's inner core. Nature, 404(6775), 273-275. 10.1038/35005059

Vinnik, L., Romanowicz, B., Le Stunff, Y., \& Makeyeva, L. (1995). Seismic anisotropy in the D "layer. Geophysical Research Letters, 22(13), 1657-1660.

Vinnik, L. P., Farra, V., \& Romanowicz, B. (1989). Observational evidence for diffracted SV in the shadow of the Earth's core. Geophysical Research Letters, 16(6), 519-522. Vinnik, L. P., Makeyeva, L. I., Milev, A., \& Usenko, A. Y. (1992, décembre). Global patterns of azimuthal anisotropy and deformations in the continental mantle. Geophysical Journal International, $111(3), 433-447$. 10.1111/j.1365-246X.1992.tb02102.x

Virieux, J., \& Operto, S. (2009). An overview of full-waveform inversion in exploration geophysics. Geophysics, 74(6), WCC1-WCC26.

Wookey, J., Kendall, J.-M., \& Rümpker, G. (2005, juin). Lowermost mantle anisotropy beneath the north Pacific from differential S-ScS splitting. Geophysical Journal International, 161(3), 829-838. 10.1111/j.1365-246X.2005.02623.x

(C)2019 American Geophysical Union. All Rights Reserved. 
Wu, R., \& Toksöz, M. (1987). Diffraction tomography and multisource holography applied to seismic imaging. Geophysics, 52(1), 11-25.10.1190/1.1442237

Wu, R.-S., \& Aki, K. (1985). Scattering characteristics of elastic waves by an elastic heterogeneity. Geophysics, 50(4), 582-595.

Wu, Z., \& Alkhalifah, T. (2017). An efficient Helmholtz solver for acoustic transversely isotropic media. Geophysics, 83(2), 1-20.

Xu, S., Stovas, A., \& Sripanich, Y. (2018, janvier). An anelliptic approximation for geometric spreading in transversely isotropic and orthorhombic media. GEOPHYSICS, 83(1), C37-C47. 10.1190/geo2017-0038.1

Yu, W. (2016, juillet). Detectability of temporal changes in fine structures near the inner core boundary beneath the eastern hemisphere. Geophysical Research Letters, 43(13), 2016GL069664. 10.1002/2016GL069664

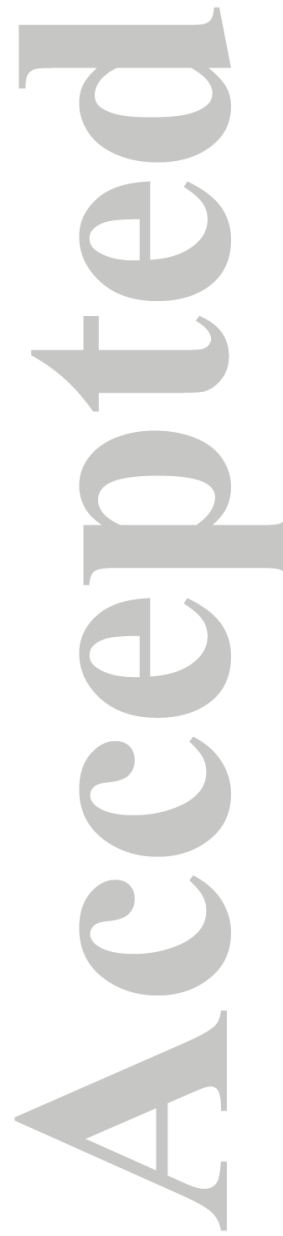




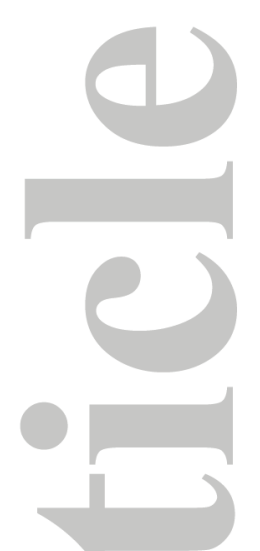

Table 1. Principally irresolvable linear combinations of orthorhombic parameters, number of resolvable parameters, and possible set of parameters that could be resolved from the $C_{i j}, \rho$ parameters for different types of scattering recorded under perfect illumination.

\begin{tabular}{|c|c|c|c|}
\hline Data: & $P-P$ & $S H-S H$ & $S V-S V$ \\
\hline Nulls VTI: & $2 \mathbf{C}_{12}-\mathbf{C}_{66} \equiv \gamma_{1}$ & $V_{p}$ \\
& $\mathbf{C}_{11}+\mathbf{C}_{12}+\mathbf{C}_{22}-\mathbf{C}_{33}+\rho$ & $\varepsilon_{1}$ & $\varepsilon_{1}$ \\
& $V_{s}-8 \varkappa \eta_{1} \equiv 2 \mathbf{C}_{13}+\mathbf{C}_{55}+2 \mathbf{C}_{23}+\mathbf{C}_{44}$ & $\eta_{1}$ & $\boldsymbol{\gamma}_{1}$ \\
\hline Nulls ORT: & & $\varepsilon_{d}+\delta_{3} \equiv 2 \mathbf{C}_{22}+\mathbf{C}_{12}$ & \\
& $2 \mathbf{C}_{23}+\mathbf{C}_{44} \equiv 8 \varkappa \boldsymbol{\eta}_{d}+\gamma_{d}$ & $\mathbf{C}_{23} \equiv \eta_{d}$ & $\lambda$ \\
& & & $\varepsilon_{1}$ \\
& & & $\varepsilon_{d} \propto \boldsymbol{\delta}_{3}$ \\
\hline \# par.: & 6 & 4 & 6 \\
Set VTI: & $V_{p}, \varepsilon_{1}, \eta_{1}$ & $V_{s}, \gamma_{1}$ & $\rho, V_{s}, \eta_{1}$ \\
\hline Set ORT: & "acoustic, $\rho=$ const" & $V_{s}, \varepsilon_{d}, \gamma_{1}, \gamma_{d}$ & $\rho, V_{s}, \varepsilon_{d}, \eta_{1}, \eta_{d}, \gamma_{d}$ \\
\hline
\end{tabular}

(C)2019 American Geophysical Union. All Rights Reserved. 


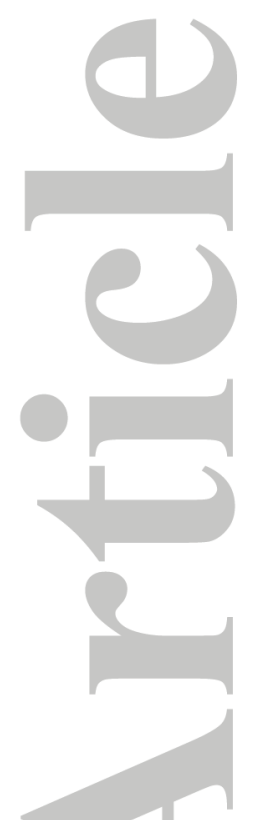

Table 2. Same as Table 1, but for converted waves and their combinations with $P$ waves.

\begin{tabular}{|c|c|c|c|c|c|}
\hline Data: & $P-S V$ & $S V-S H$ & $P-S H$ & $P-P, P-S V$ & $P-P, P-S V, P-S H$ \\
\hline Nulls VTI: & $\gamma_{1}$ & all VTI & all VTI & $\gamma_{1}$ & $\gamma_{1}$ \\
& $V_{p} \equiv \lambda$ & & & & - \\
Nulls ORT: & - & $2 \varepsilon_{d}+\delta_{3}$ & - & - & 9 \\
\hline \# par.: & 8 & 3 & 4 & 9 & \\
\hline
\end{tabular}

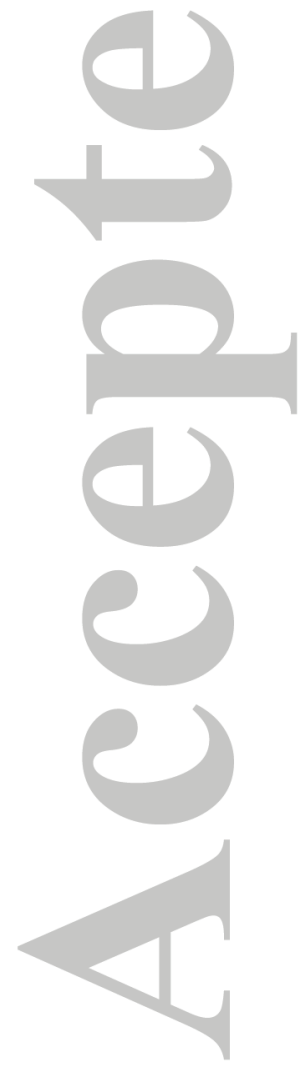

(C)2019 American Geophysical Union. All Rights Reserved. 


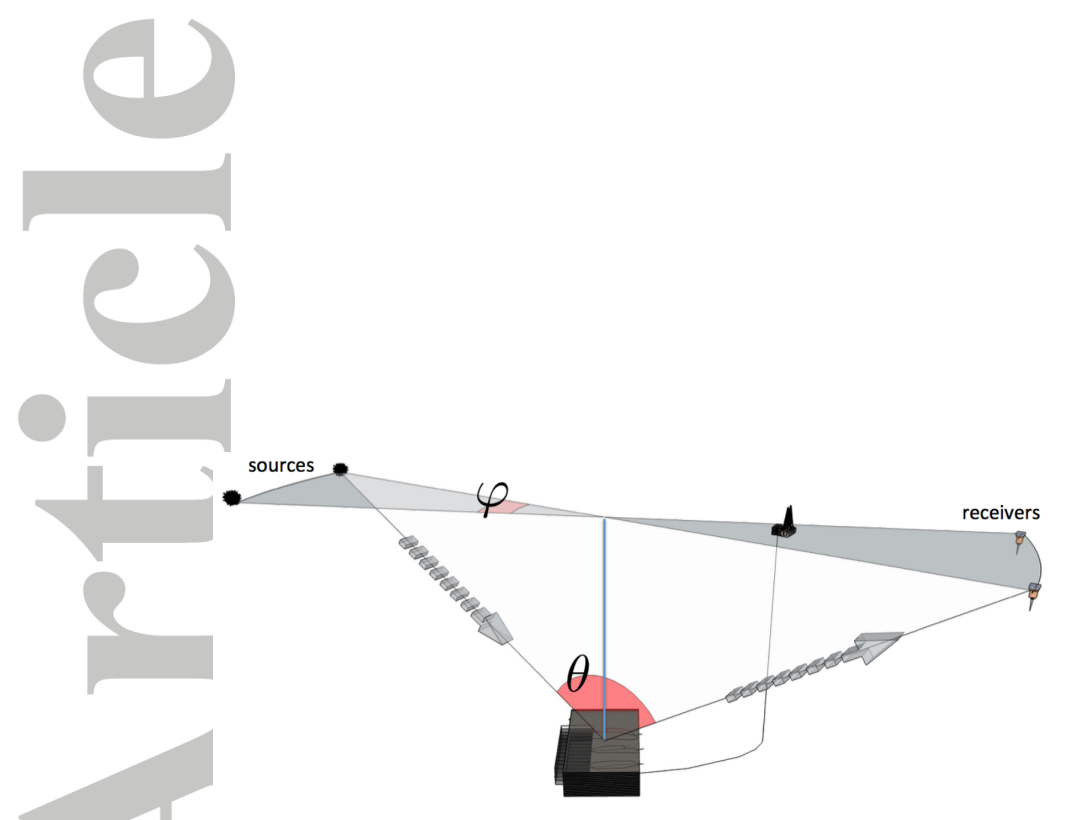

(a)

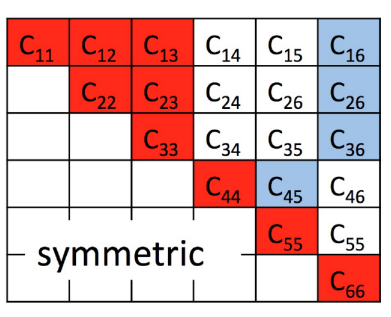

(b)

Figure 1. Orthorhombic reservoir. a) Anisotropic fractured reservoir (small black box with vertical planes) in isotropic background. Arrows indicate incident and scattered waves, $\theta$ denotes the incidence angle and $\varphi$ the azimuth of the reflection plane w.r.t. the symmetry plane of the reservoir. b) Elements of stiffness matrix in Voigt notation (Babuska \& Cara, 1991). Only red and blue cells are non-zero for monoclinic media with horizontal $\left(x_{1}-x_{2}\right)$ plane of symmetry. In orthorhombic media only nine (in red) parameters are non-zero (if the symmetry planes are $\left.x_{1}-x_{2}, x_{1}-x_{3}, x_{2}-x_{3}\right)$.

(C)2019 American Geophysical Union. All Rights Reserved. 


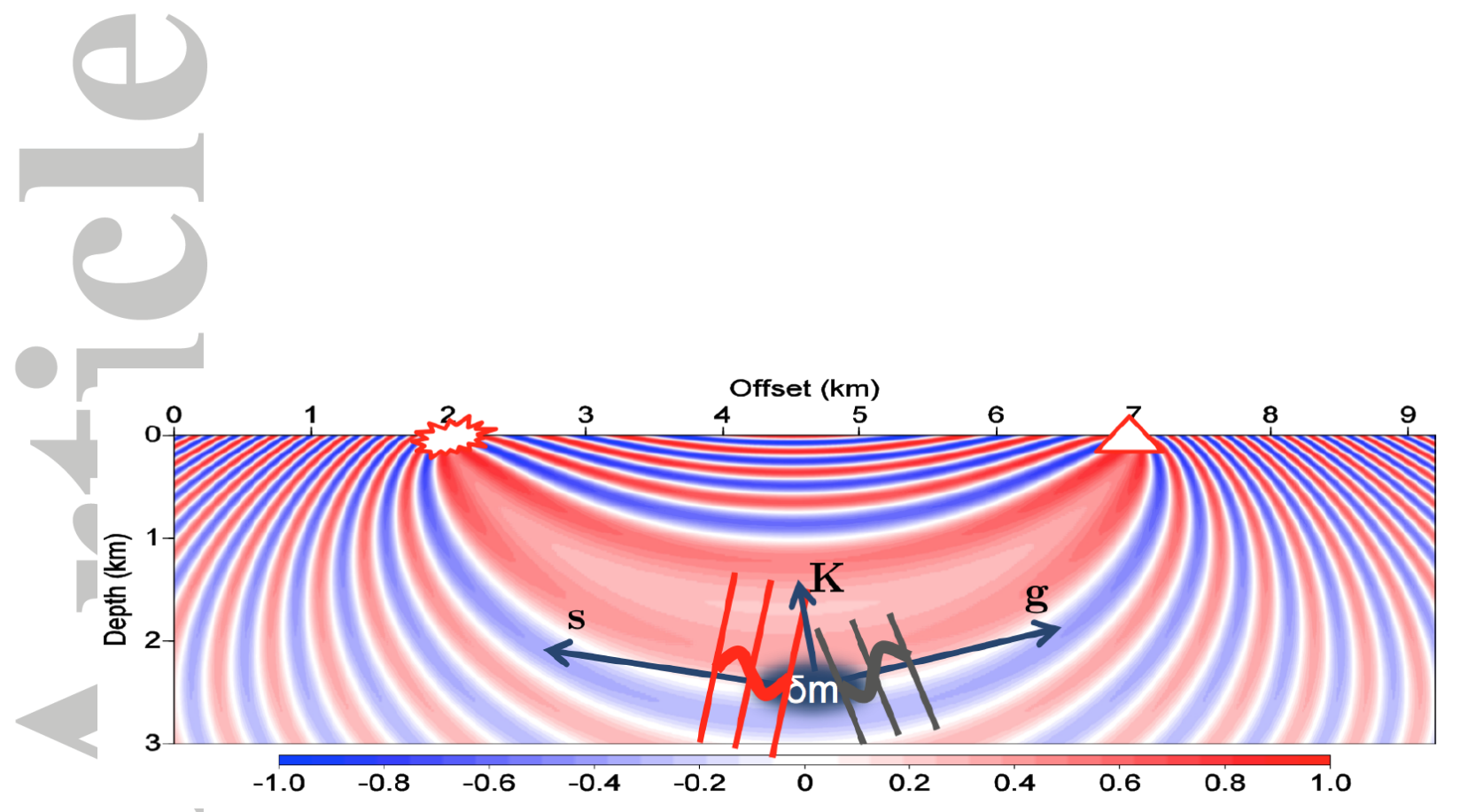

Figure 2. Sensitivity kernel for a single source-receiver pair for FWI in the frequency domain in a linearly increasing velocity background (Alkhalifah, 2015 ; Kazei et al., 2016). Source is indicated as an explosion and receiver as a triangle. On the wide red wavepath of diving wave the update can only be smooth, wavenumbers in the gradient $\mathbf{K}=\omega(\mathbf{s}+\mathbf{g})$ are very small. With a decrease in the scattering angle (the angle between slowness vectors towards the source s and receiver $\mathbf{g}$ ), the resolution length $L \propto|\mathbf{K}|^{-1}$ also decreases, which results in more wiggly structure of the gradient away from the wavepath.

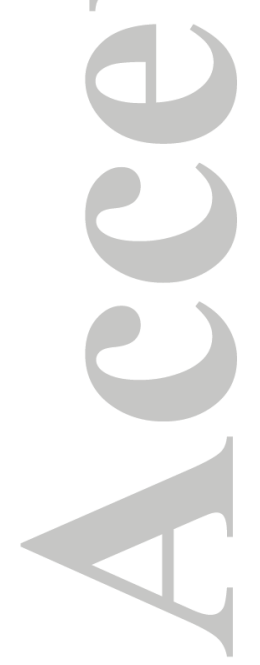




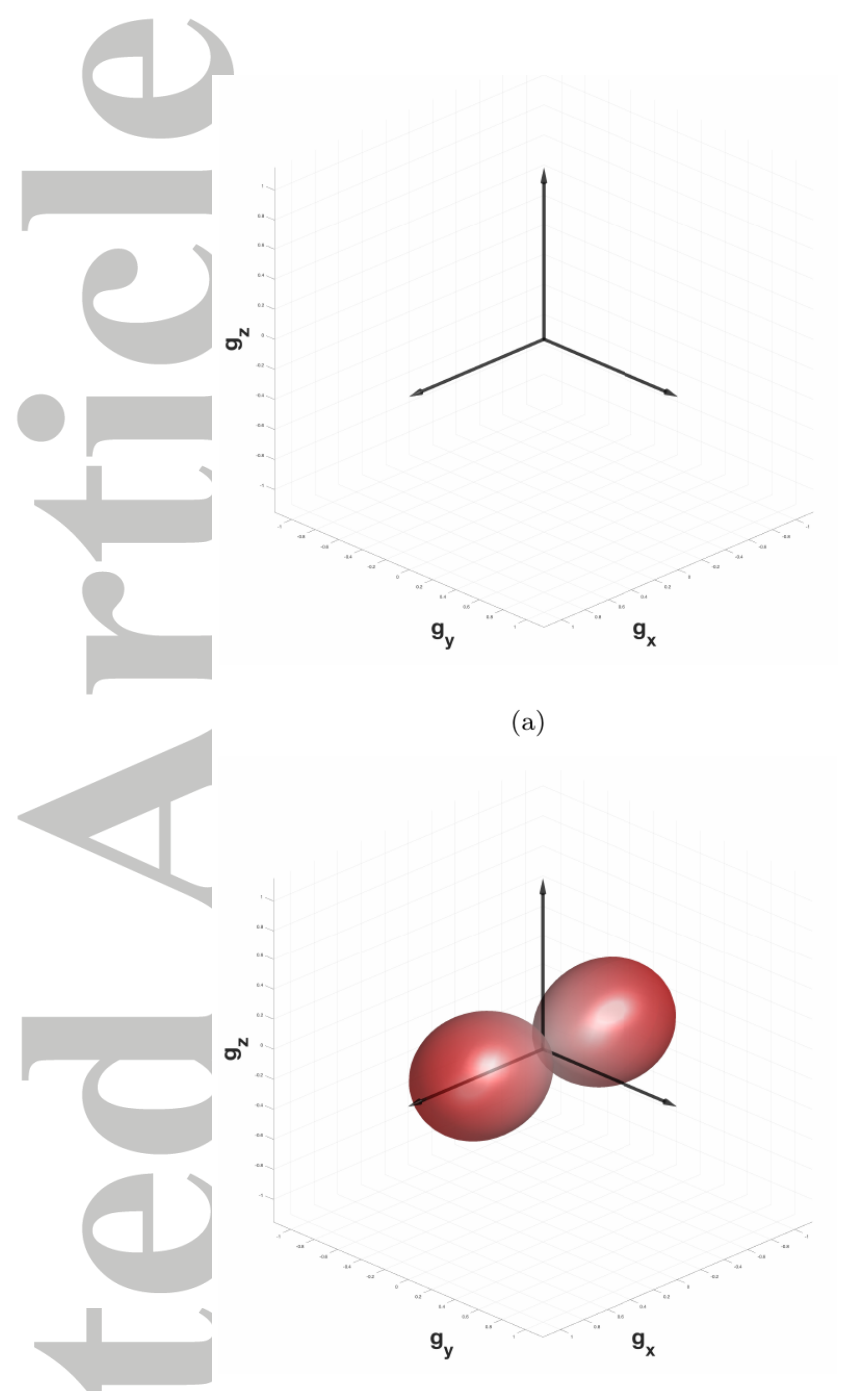

(c)

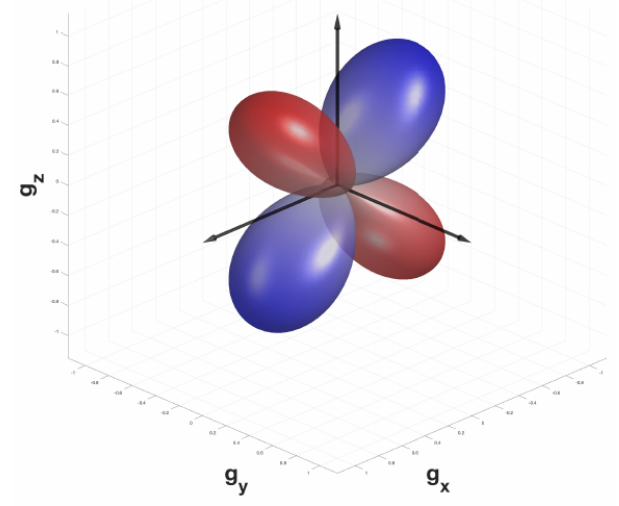

(b)

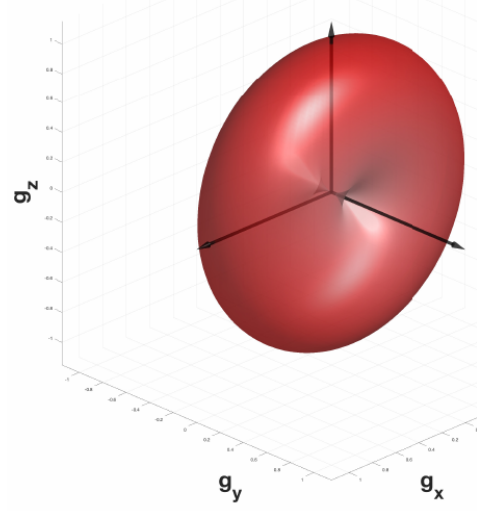

(d)

Figure 3. Diffraction-based radiation pattern for $P-P$ scattering and perturbation in $C_{55}$ and $C_{13}$ for vertical and oblique $\left(\mathbf{s}=\frac{1}{\sqrt{3} V_{p}}(1,1,1)^{T}\right)$ incidence. $C_{55}$ does not scatter vertically incident wayes - (a), but scatters for out of symmetry plane incidence - (b). $C_{13}$ works like an $\mathrm{X}$-axis expansion source for vertical incidence and like expansion source in $\mathrm{XZ}$ plane for oblique incidence $-(d)$.

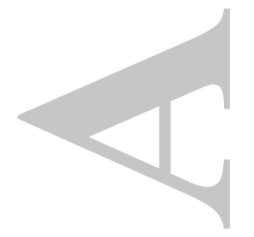

(C)2019 American Geophysical Union. All Rights Reserved. 


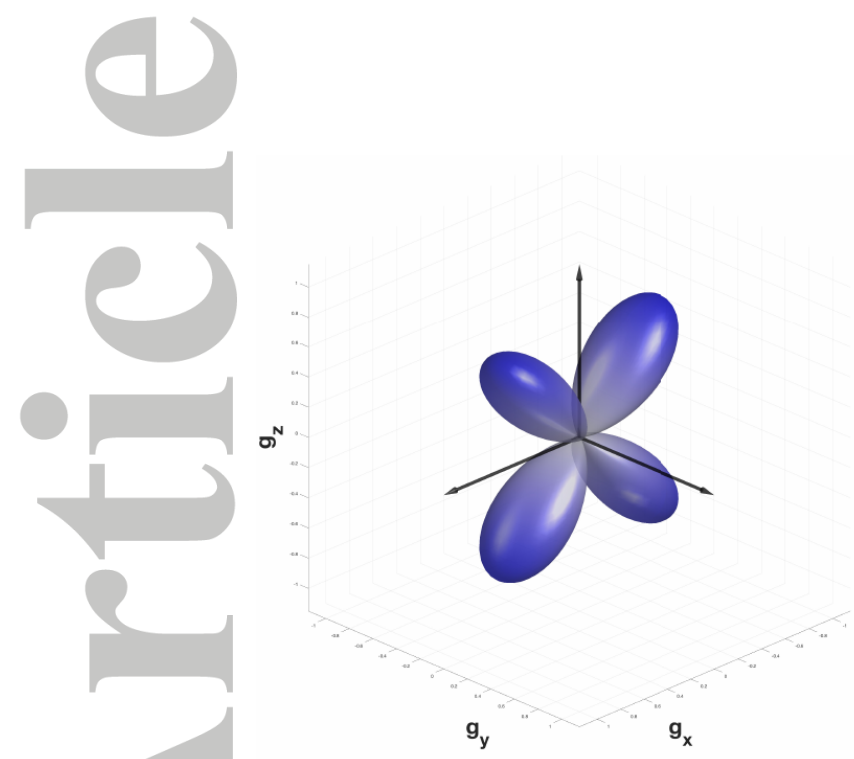

(a)

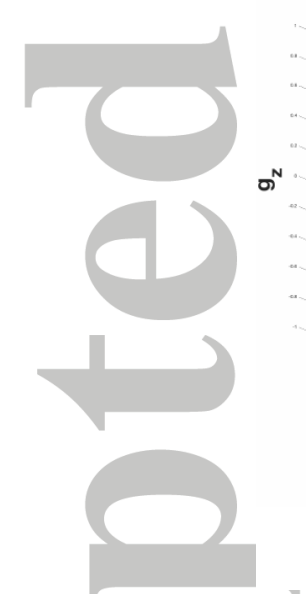

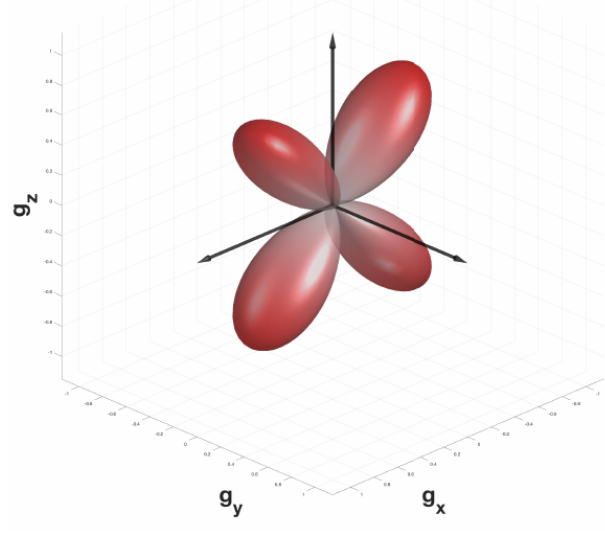

(b)

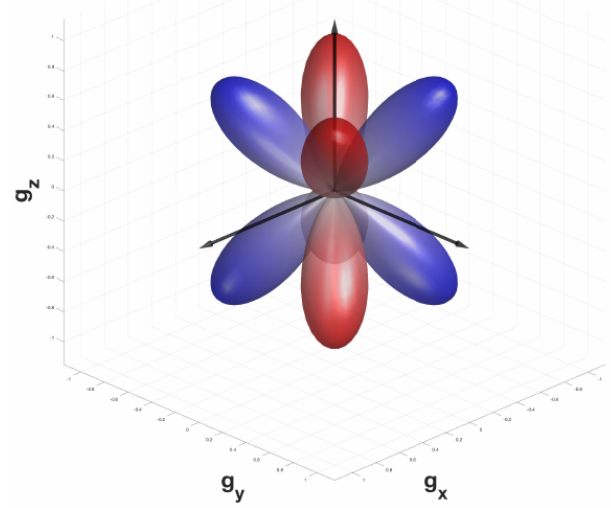

(d)

Figure 4. Reflection-based radiation patterns for $P-P$ scattering and $C_{55}-(\mathrm{a}), C_{13}-(\mathrm{b})$, $C_{16}-(\mathrm{c}), C_{36}-(\mathrm{d})$. Just by looking at the radiation patterns we can state that $C_{13}$ and $C_{55}$ can not be determined together at high precision as they scatter in a very similar if not identical way. 


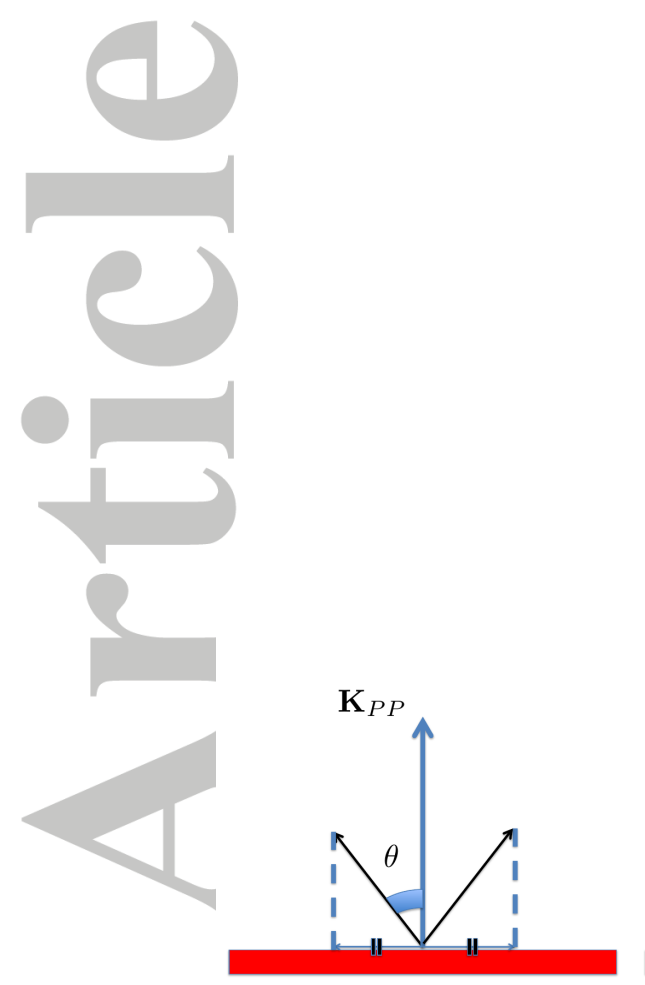

(a)

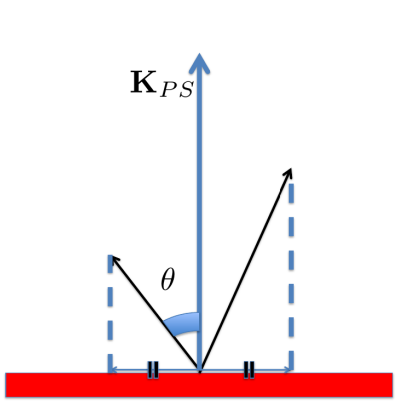

(b)

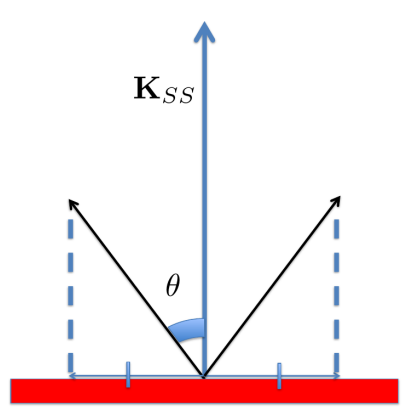

(c)

Figure 5. For the same incident angle and same temporal frequency different modes provide illumination to different vertical wavenumbers. (a) $P-P$ waves illuminate the lowest wavenumbers (good for FWI), (c) $S-S$ waves - the highest wavenumbers (good for migration), and (b) $P-S$ waves - the intermediate wavenumbers.

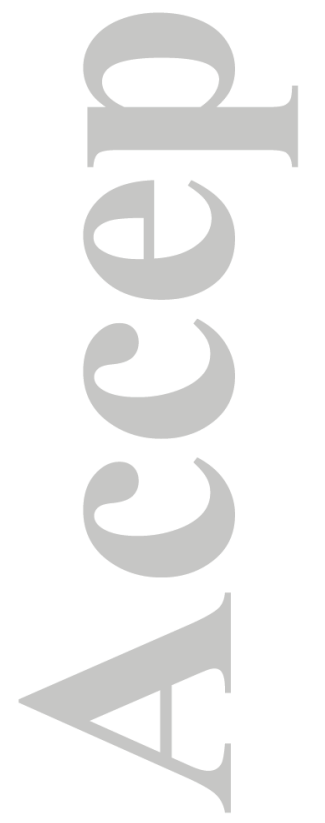

(C)2019 American Geophysical Union. All Rights Reserved. 


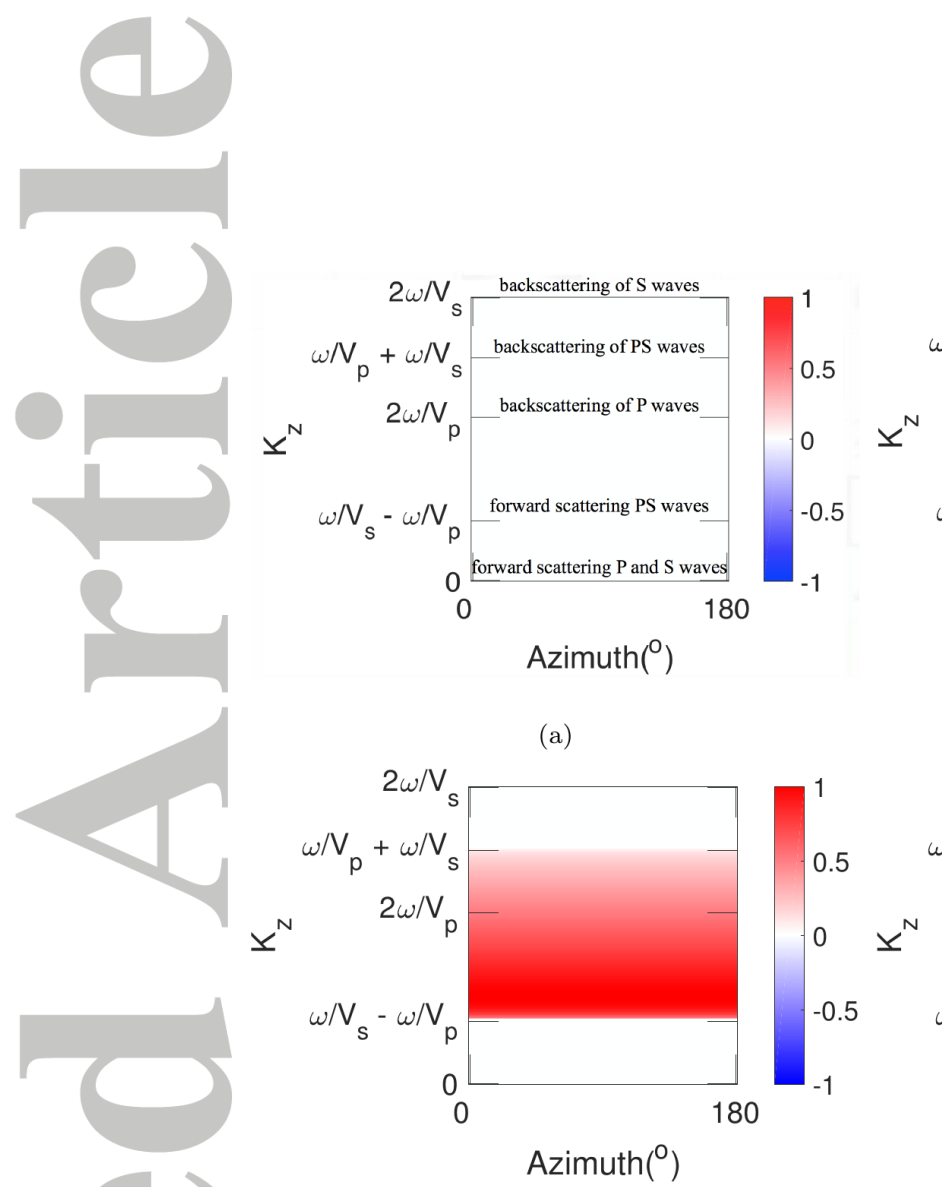

(c)

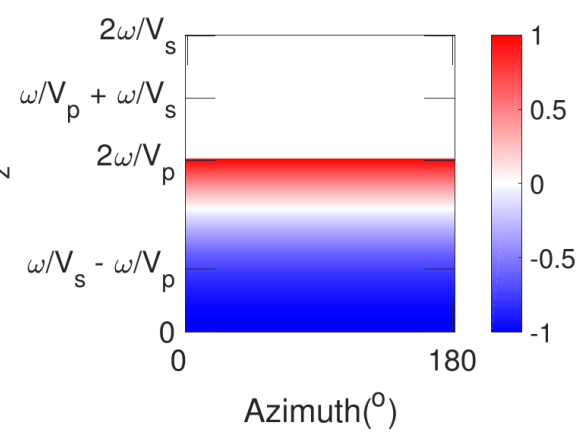

(b)

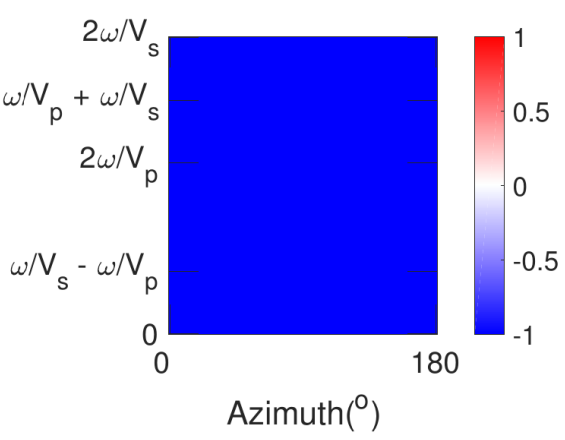

(d)

Figure 6. Scattering of different wave types. (a) Wavenumber/resolution limitations for different types of scattering on the same scale. (b),(c),(d) Scattering in the spectral domain for density for $P \neg P, P-S V, S H-S H$ waves. $P$ waves cannot resolve intermediate wavenumbers in the spectrum of density - see the white stripe in the middle of (b); $P-S V$ waves can close this gap (c). Finally, $S H-S H$ waves can in principle illuminate the whole spectrum of density. 


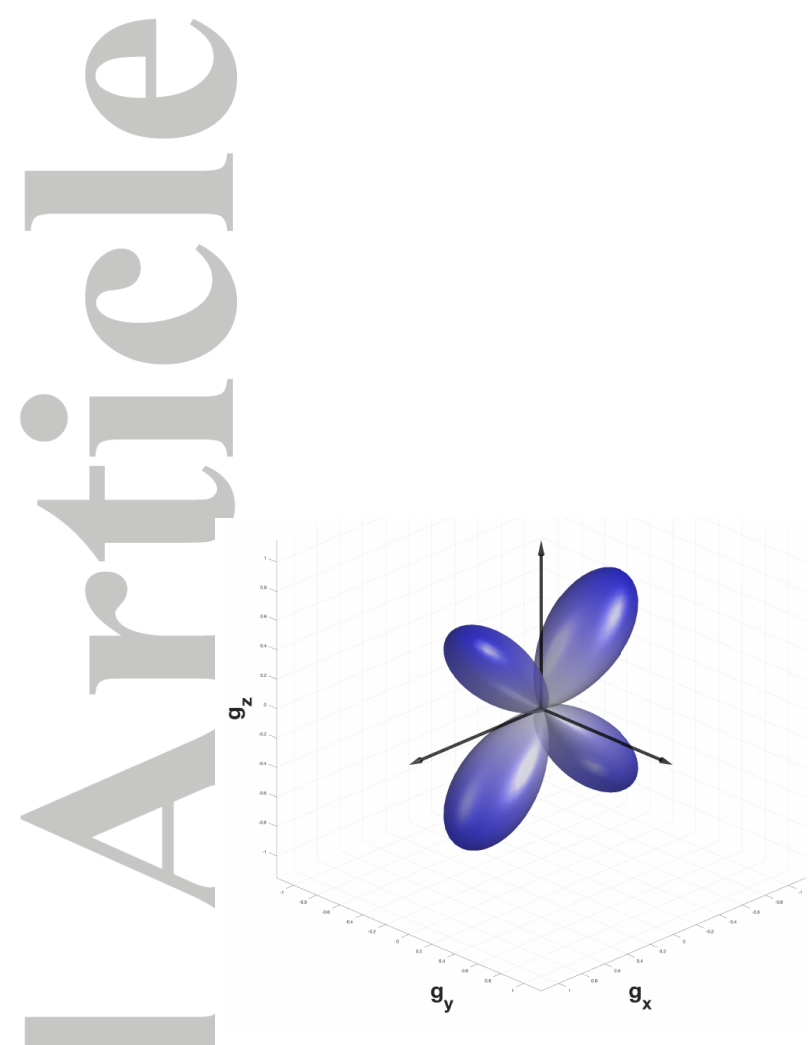

(a)

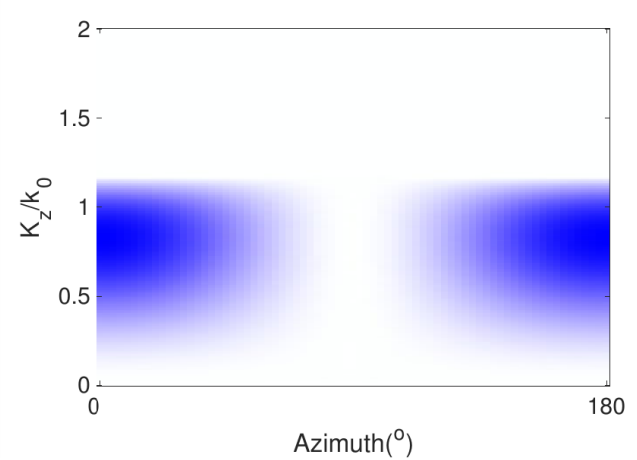

(b)

Figure 7. (a) The reflection-based radiation pattern for $C_{55}$ and (b) its remapping into the spectral domain. The normalized wavenumber $\|\mathbf{K}\| \propto \cos \theta_{\max }$. In all the patterns, the amplitude sign is indicated by color: red corresponds to a positive reflection coefficient, i.e., no phase flip after scattering; blue shows a negative reflection or scattering coefficient.

(C)2019 American Geophysical Union. All Rights Reserved. 


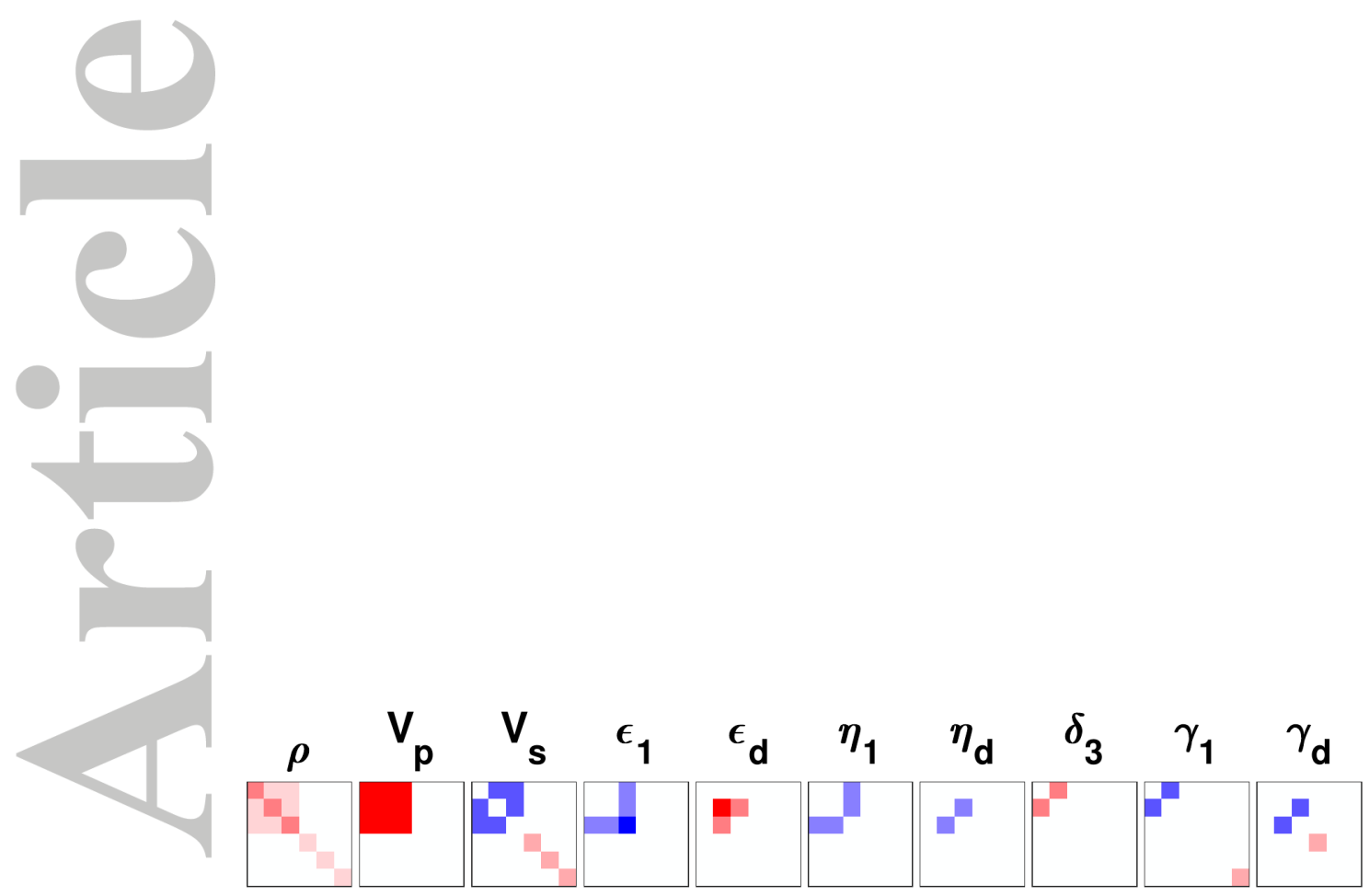

Figure 8. Partial derivatives of $C_{i j}$ parameters w.r.t. hierarchical parameterization parameters. Throughout the paper, the Poisson ratio is assumed to be equal to $0.25\left(V_{0 s} / V_{0 p}=\varkappa=\frac{1}{\sqrt{3}}\right)$, which in fact affects only the derivatives w.r.t. density.

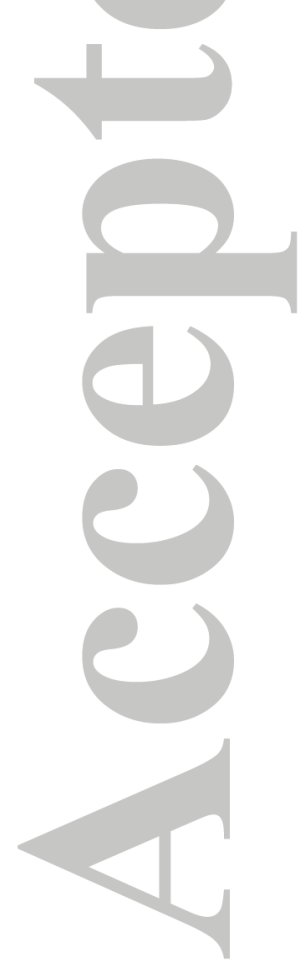




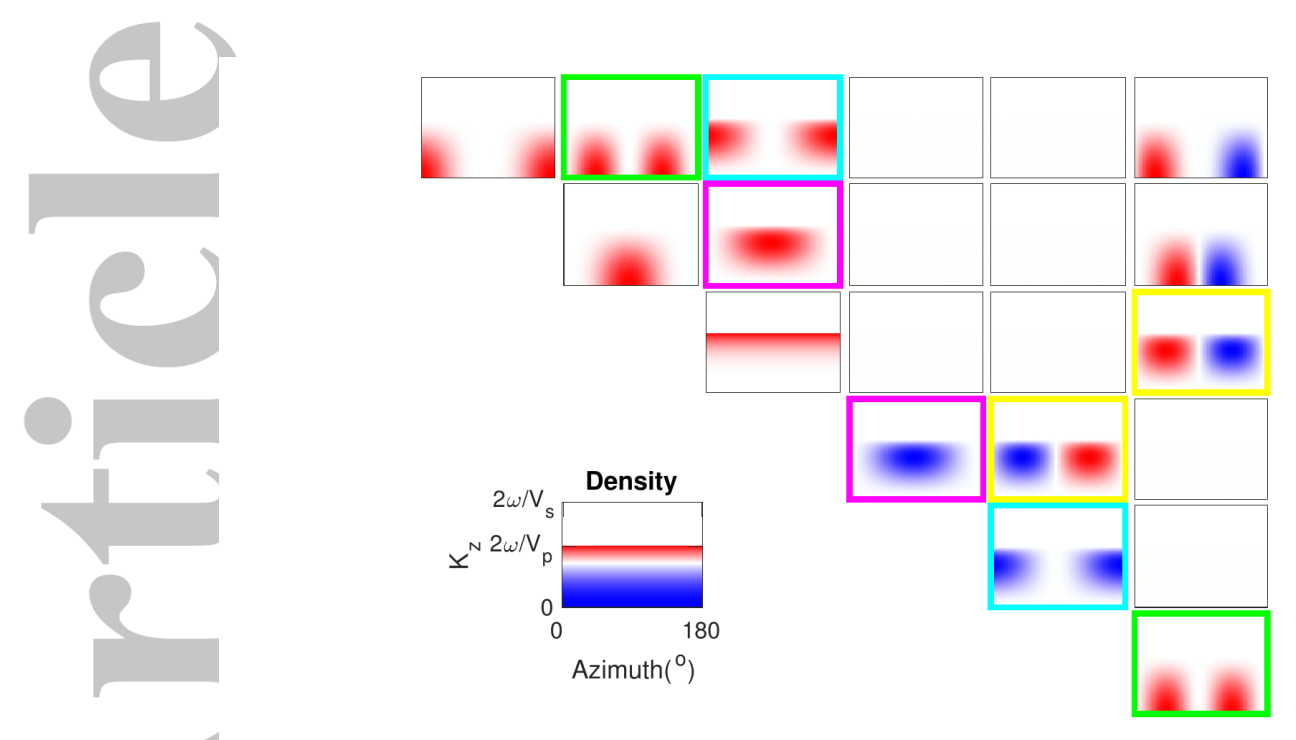

(a)

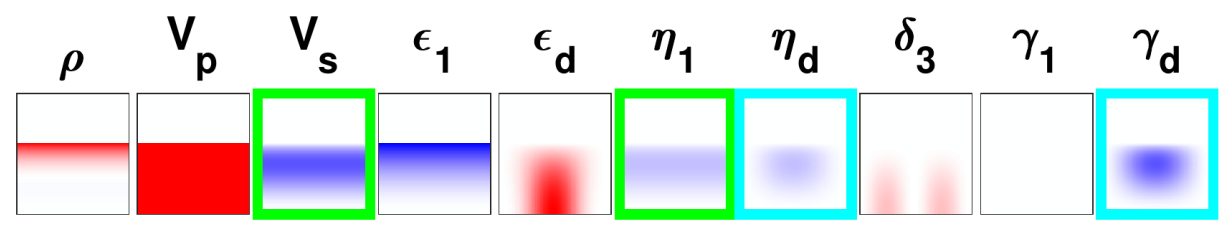

(b)

Figure 9. Spectral sensitivities to vertical wavenumbers for $P-P$ scattering on various $C_{i j}$ perturbations. Perturbations that scatter in a very similar manner are enclosed by frames of the same color. (a) Sensitivity to all $C_{i j}$ parameters and density perturbed independently. High wavenumbers for density resemble those for $C_{33}$, these are the only parameters scattering independently of the azimuth in the $C_{i j}, \rho$ parameterization. Sensitivity to all monoclinic parameters is non-zero when we start from isotropic background. Yet, $C_{13}$ is very similar to $C_{55}, C_{12}$ to $C_{66}$, and $C_{23}$ to $C_{44}$. Scattering by $C_{45}$ is similar to that by $C_{35}$. (b) In the hierarchical parameterization, $V_{s}$ is coupled to $\eta_{1}$, and $\eta_{d}$ is coupled to $\gamma_{d}$, finally, $\rho$ is very similar to $\epsilon_{1}$. The Poisson ratio is assumed to be $0.25\left(V_{p} / V_{s}=\sqrt{3}\right)$.

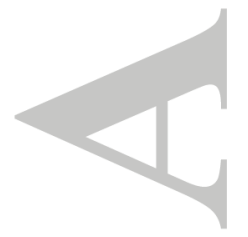

(C)2019 American Geophysical Union. All Rights Reserved. 


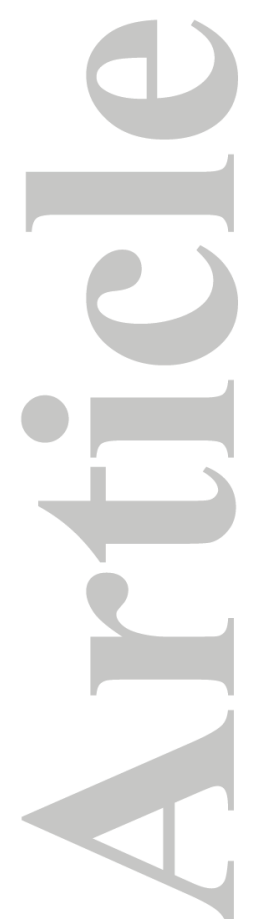

Figure 10.

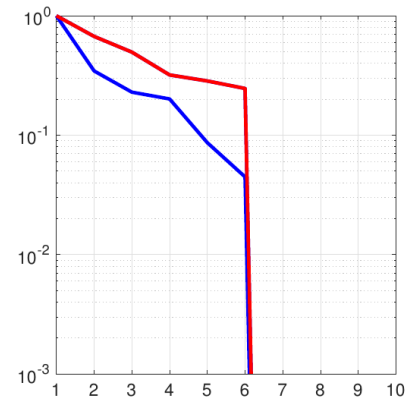

(a)

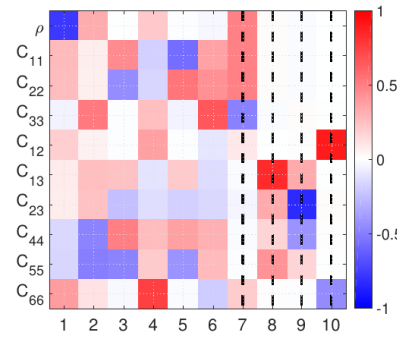

(b)

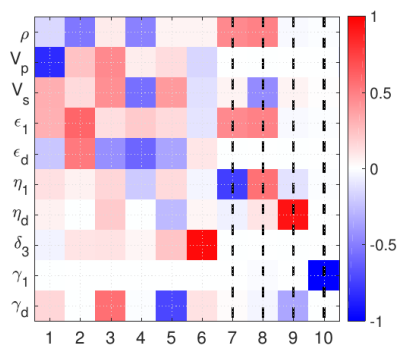

(c)

$C_{i j}, \rho$ parameterization. In Figure (a) the red line represents singular values in the standard $C_{i j}, \rho$ parameterization and the blue line represents the hierarchical parameterization. Perfect illumination - maximum opening angle is equal to $\pi$, all azimuths are available. There are six non-zero singular values. (c) Singular vectors in the hierarchical parameterization. Singular vectors corresponding to zero singular values form the null space. They are marked by vertical dashed lines. These combinations of parameter perturbations don't scatter $P-P$ waves. 

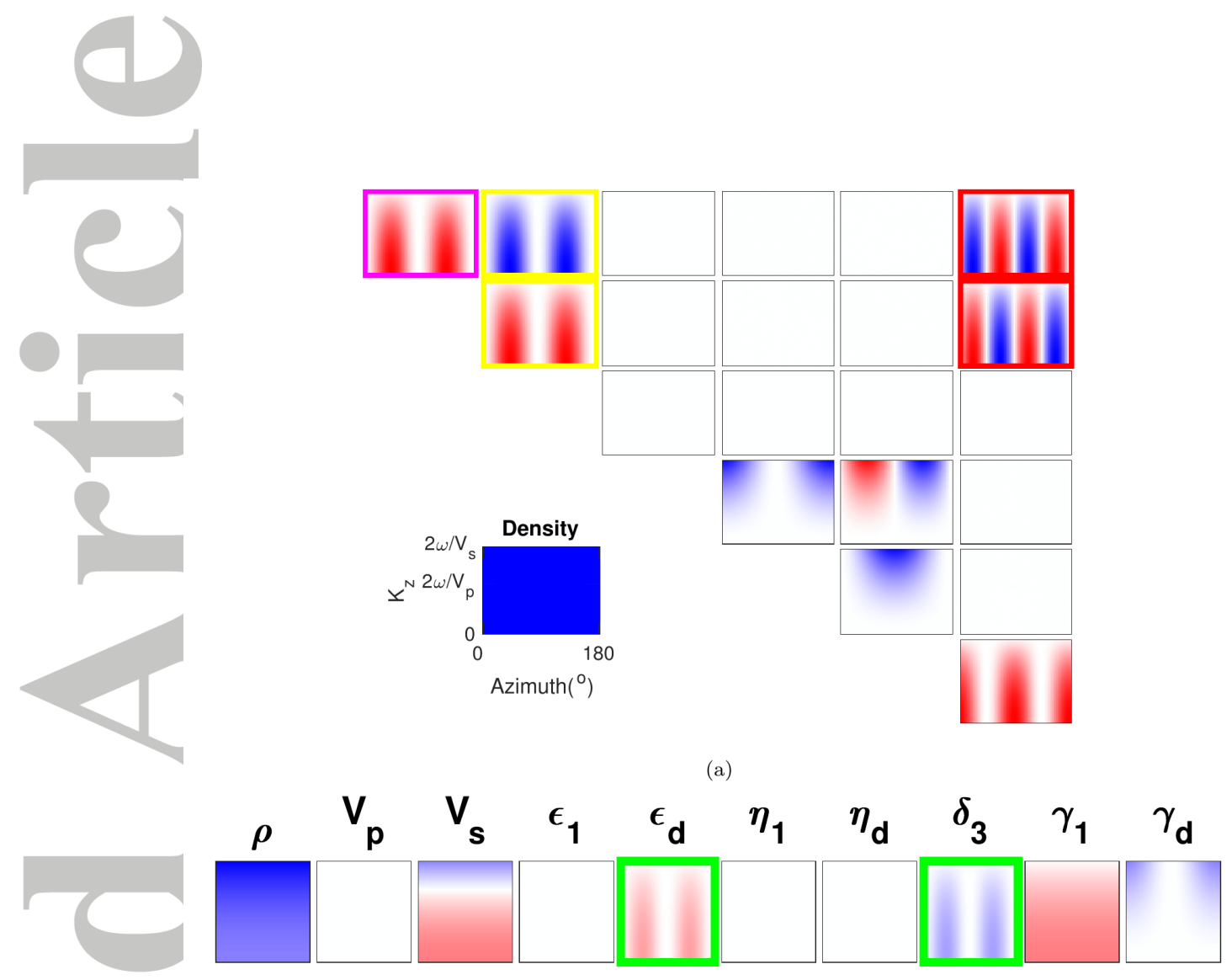

(b)

Figure 11. Same as Fig. 9, but for $S H-S H$ scattering. Scattering on density depends only on the angle between polarization vectors of incident and scattered wavefields, for $S H$ reflections from a horizontal reflector these vectors are parallel, hence the pattern is constant in $C_{i j}, \rho$ parameterization. $S H$ scattering is only sensitive to monoclinic parameters, and, thus, is non-sensitive to the perturbations of $C_{.3}$ parameters, $V_{p}, \epsilon_{1}, \eta_{1}$, and $\eta_{d}$. $C_{11}$ is coupled to both $C_{22}$ and $C_{12} . C_{16}$ is coupled to $C_{26}$.

(C)2019 American Geophysical Union. All Rights Reserved. 


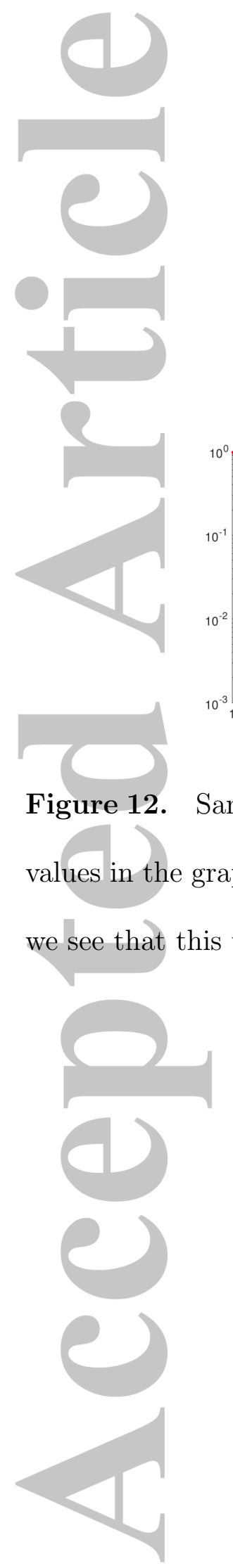

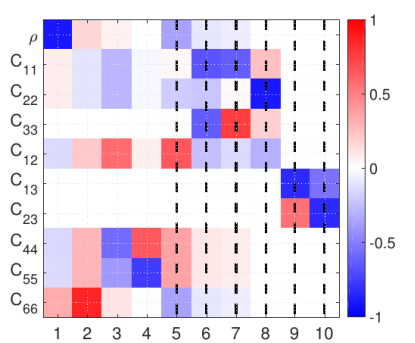

(b)

)

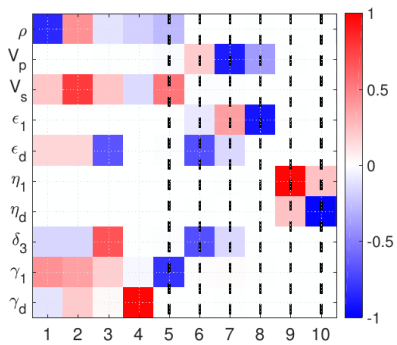

(c)

(c)

(a)

ame as Fig. 10 but for $S H-S H$ scattering. There are only three non-zero singular 

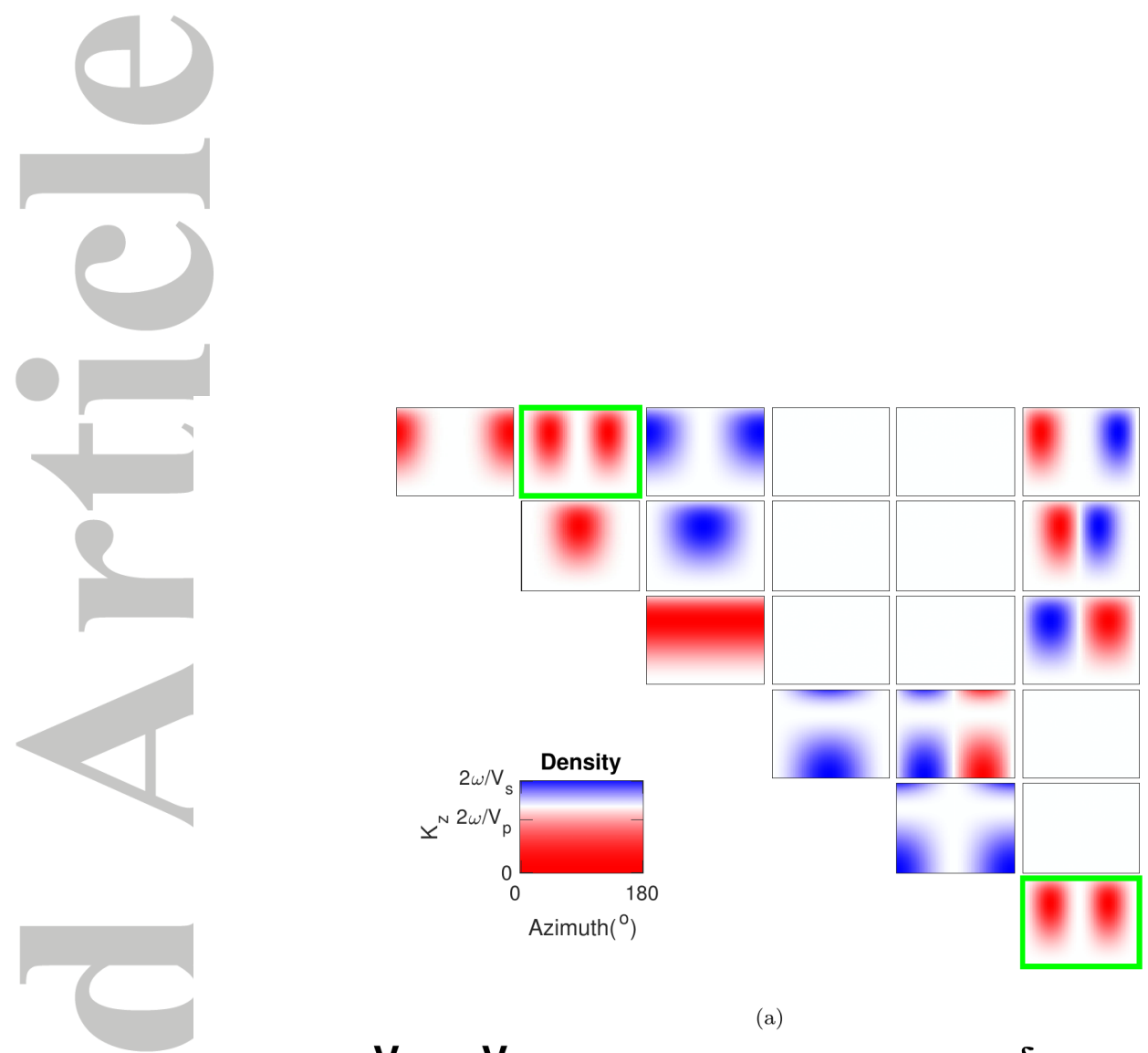

(a)

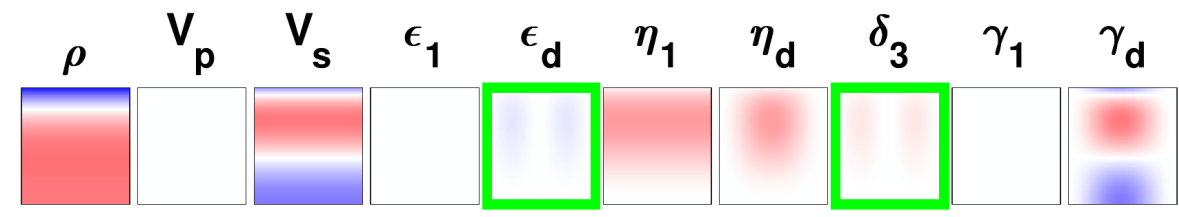

(b)

Figure 13. Same as Fig. 9, but for $S V-S V$ scattering.

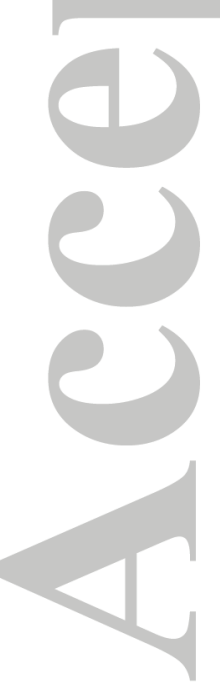

(C)2019 American Geophysical Union. All Rights Reserved. 

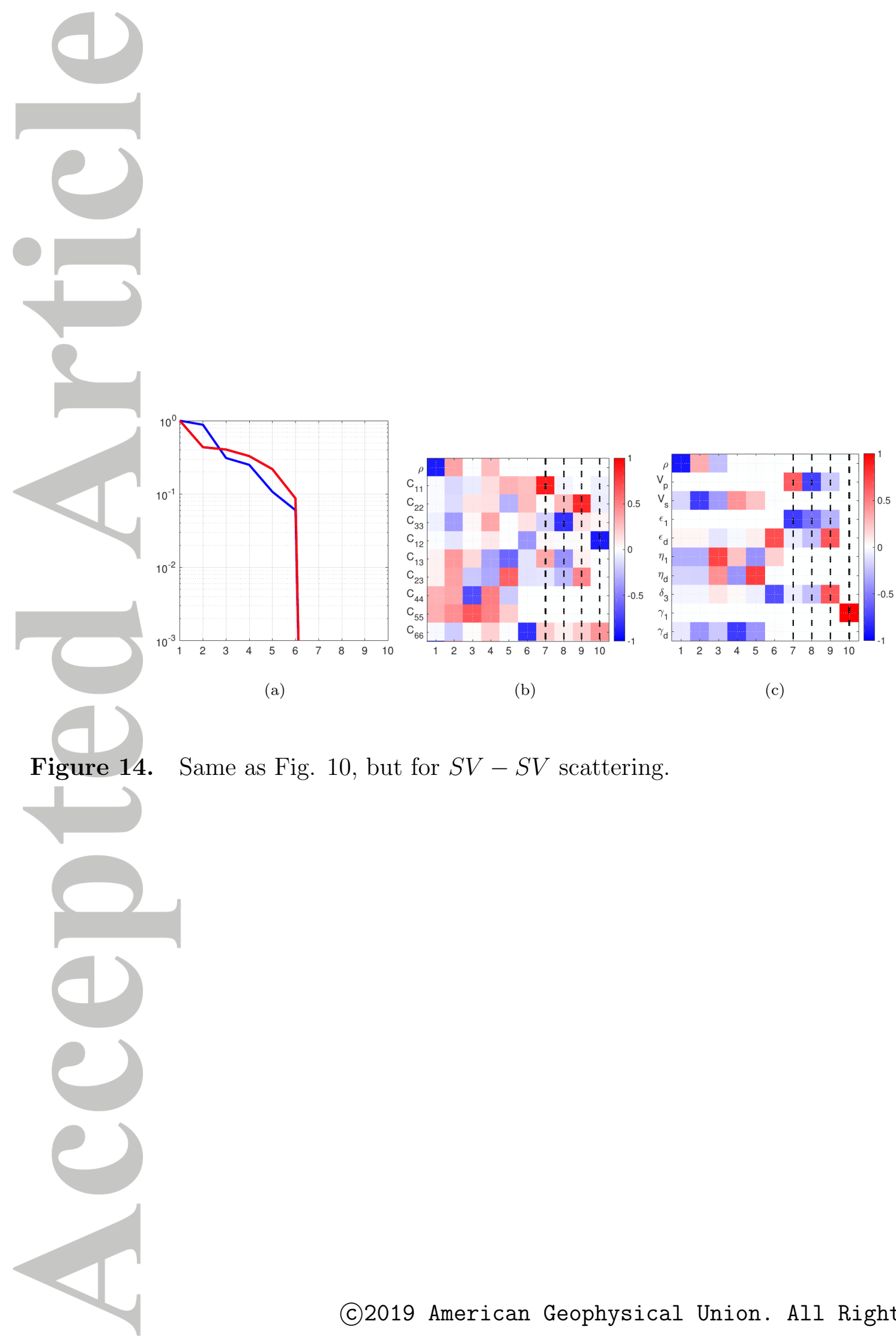

(a)

(b)

(c)

Figure 14. Same as Fig. 10, but for $S V-S V$ scattering. 


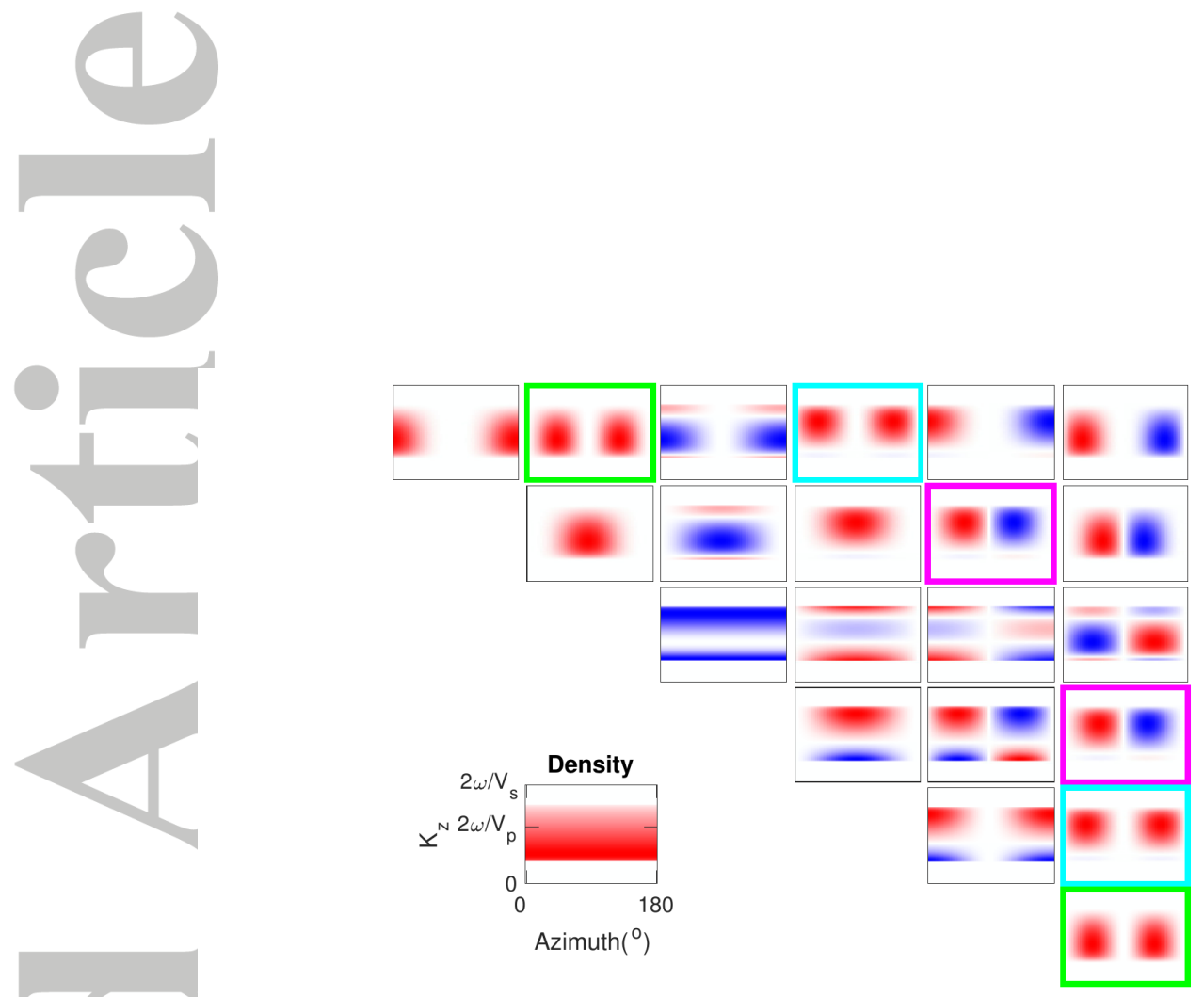

(a)

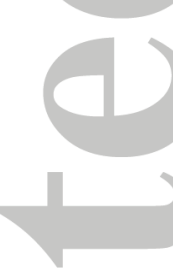

\begin{tabular}{llllllllllll}
$\rho$ & $V_{p}$ & $V_{s}$ & $\epsilon_{1}$ & $\epsilon_{d}$ & $\eta_{1}$ & $\eta_{d}$ & $\delta_{3}$ & $\gamma_{1}$ & $\gamma_{d}$ \\
& & & & & & & & & & & \\
\hline
\end{tabular}

(b)

Figure 15. Same as Fig. 9(a), but for $P-S V$ scattering. Density and $\epsilon_{1}$ scatter in a very similar way, yet visual analysis is misleading and they are distinguishable as Fig. 16(a) shows. 


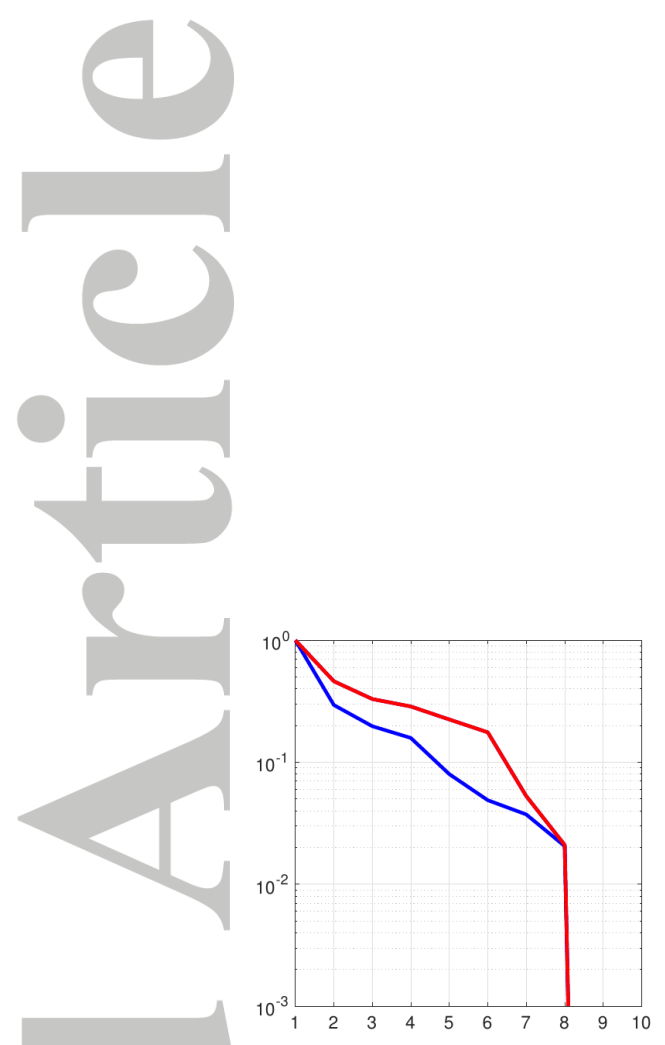

(a)

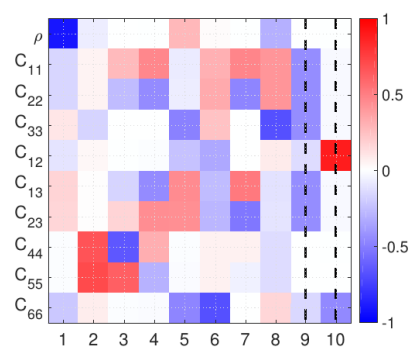

(b)

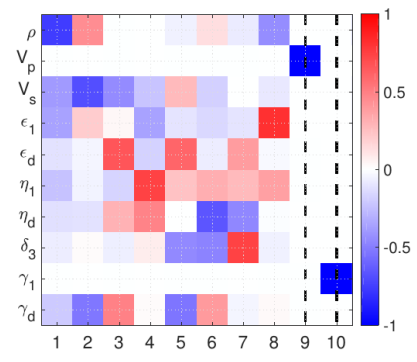

(c)

Figure 16. Same as Fig. 10, but for $P-S V$ scattering. (a) total number of non-zero singular values is eight and therefore only two parameters cannot be inverted. These are $V_{p}$ and $\gamma_{1}$ as (c) shows.

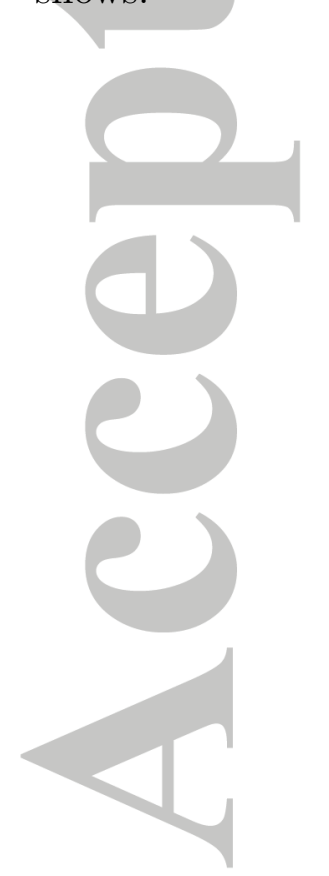



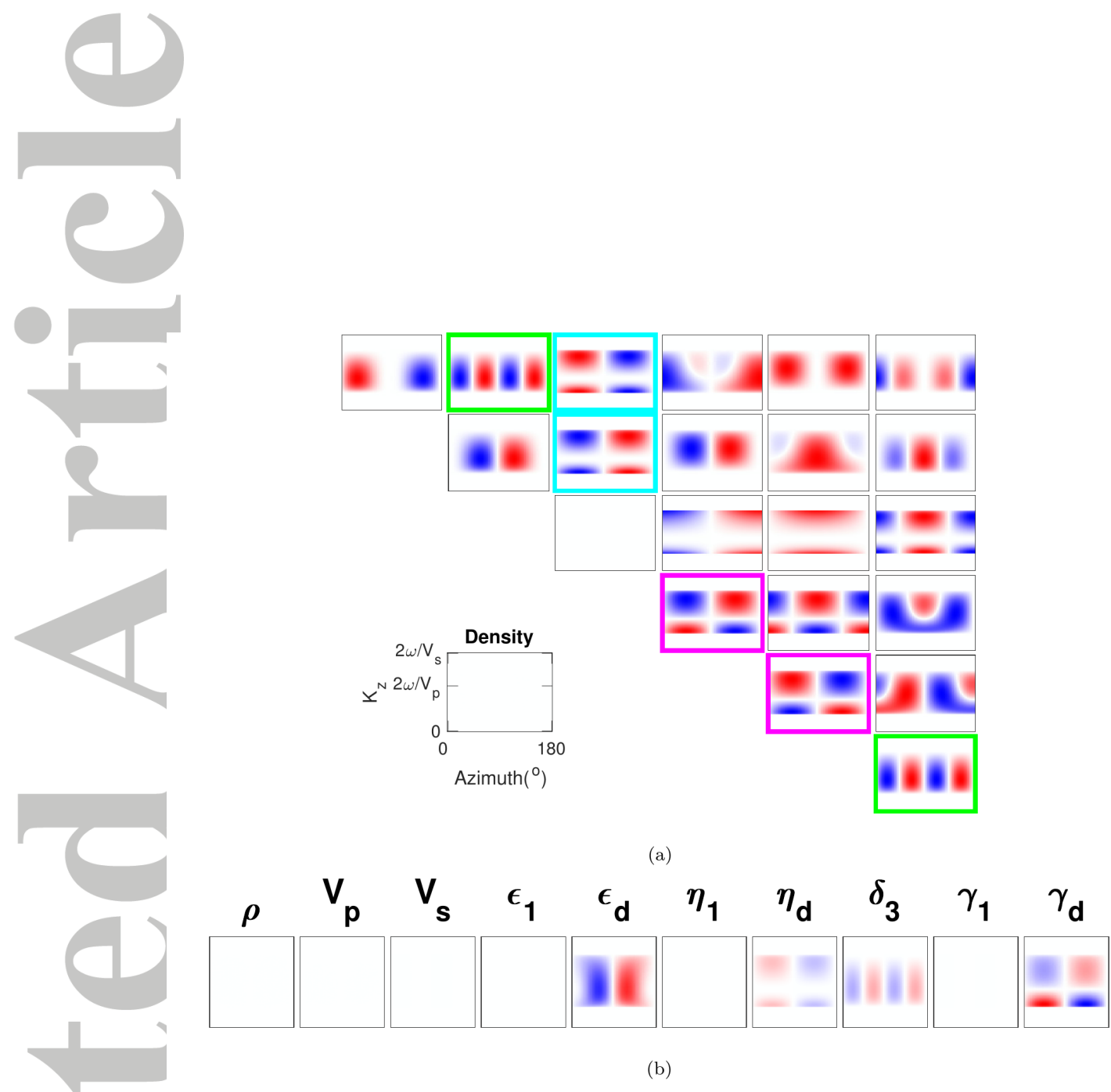

(b)

Figure 17. Same as Fig. 9, but for P-SH waves. Hierarchical parameterization shows that this type of scattering does not happen in VTI media due to simple symmetry restrictions.

(C)2019 American Geophysical Union. All Rights Reserved. 


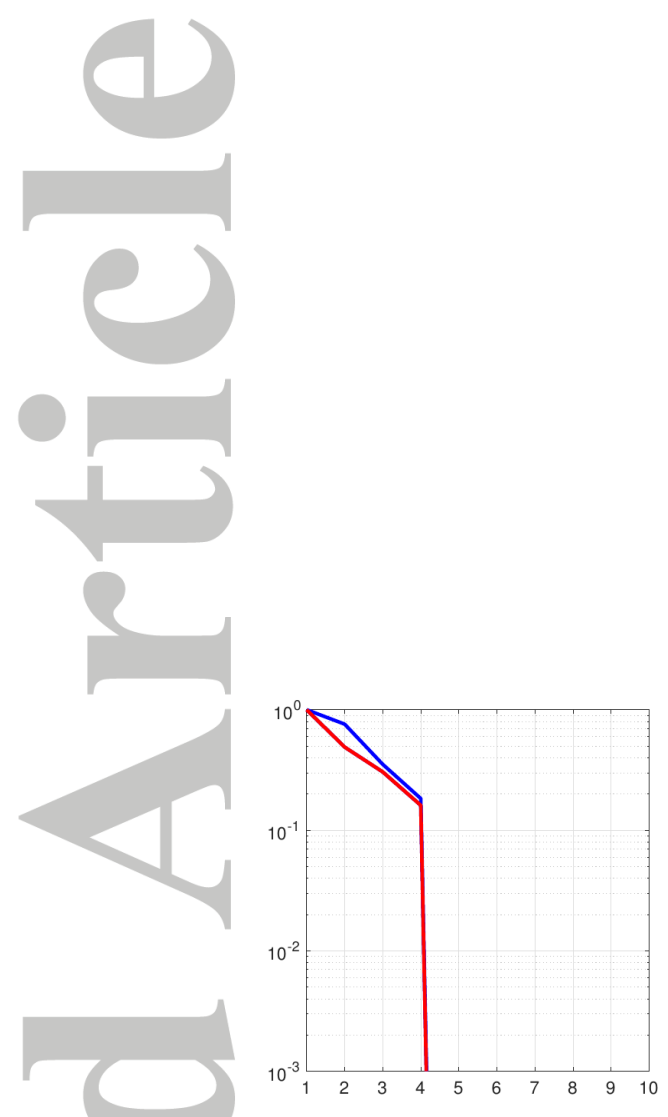

(a)

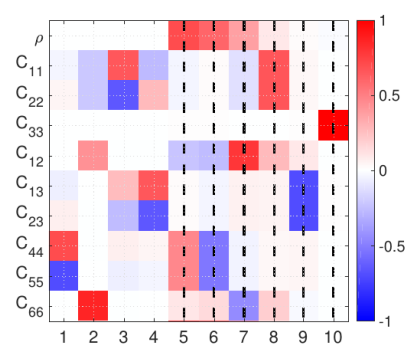

(b)

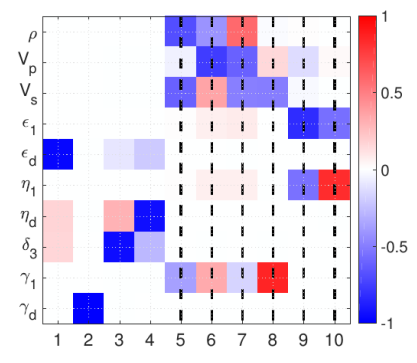

(c)

Figure 18. Same as Fig. 10, but for $P-S H$ scattering. 

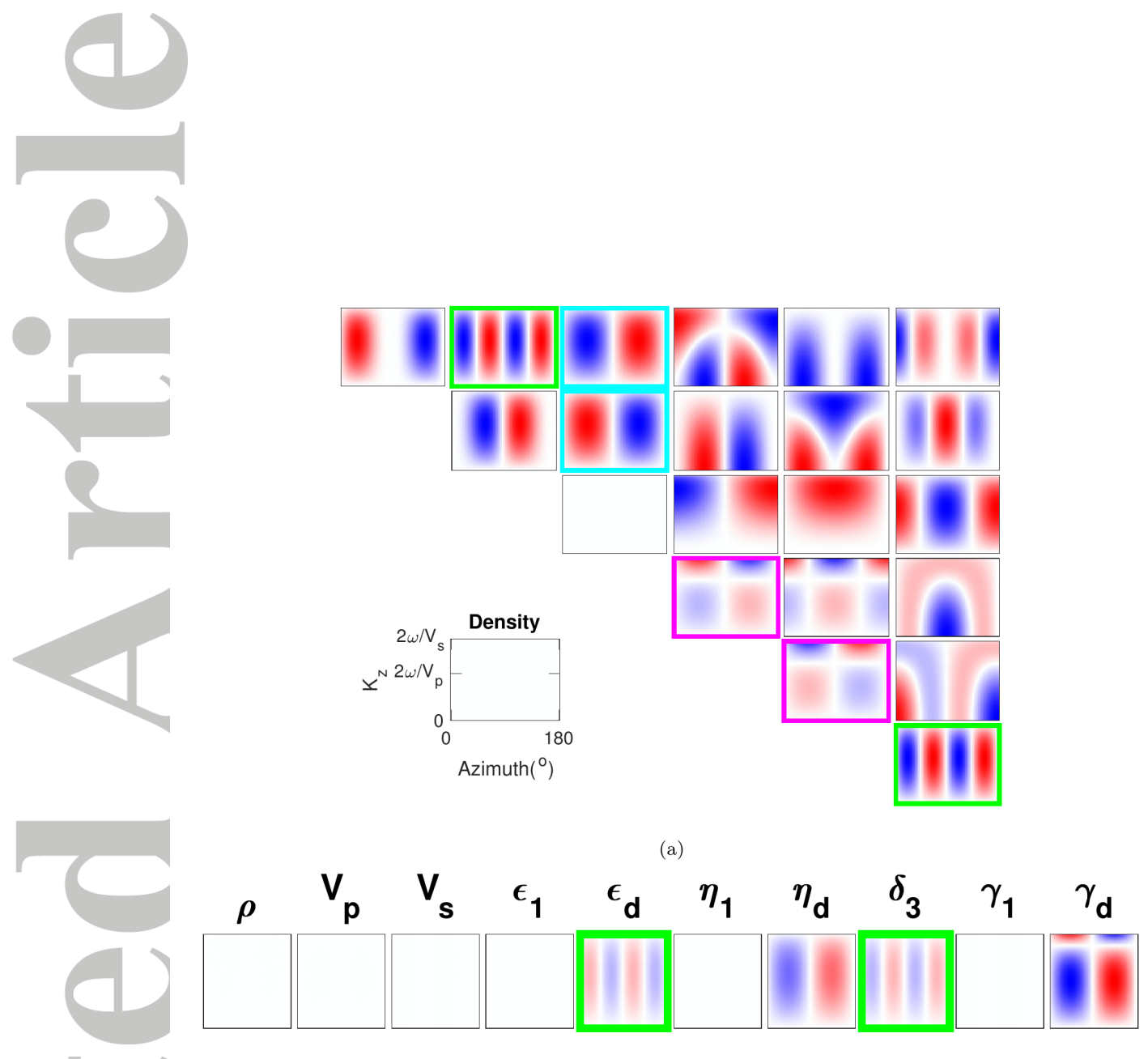

(b)

Figure 19. Same as Fig. 9, but for $S V-S H$ scattering, which is sensitive to all the $C_{i j}$ parameters except $C_{33} . S V-S H$ scattering doesn't happen in VTI media and therefore 6 VTI parameters in the hierarchical parameterization don't scatter.

(C)2019 American Geophysical Union. All Rights Reserved. 


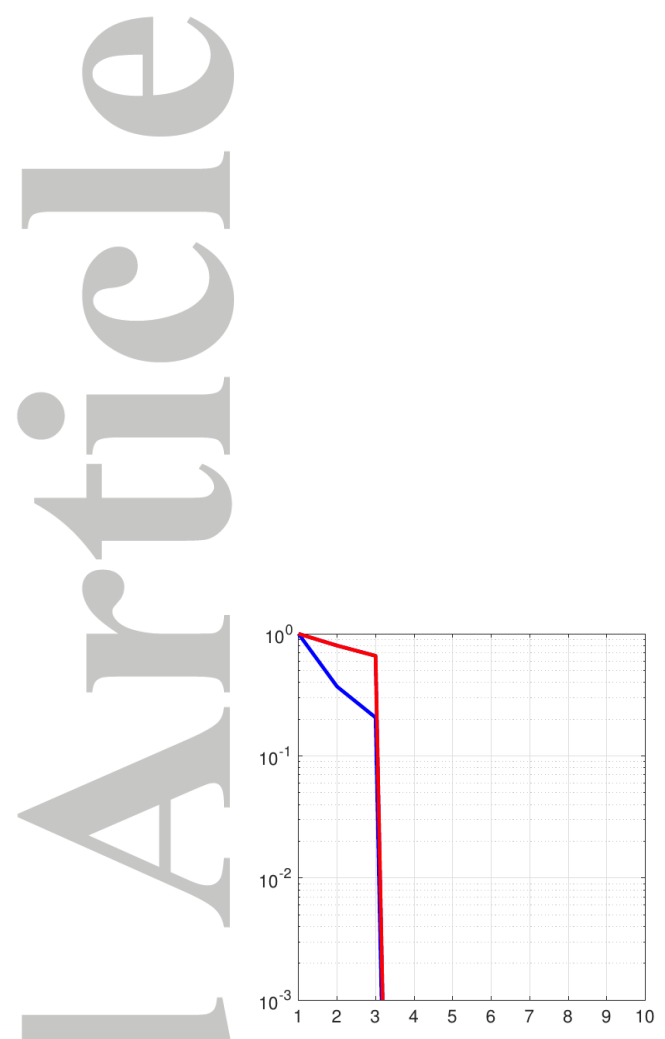

(a)

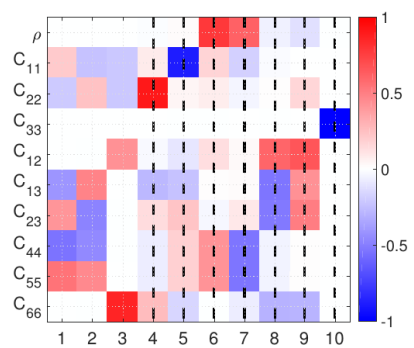

(b)

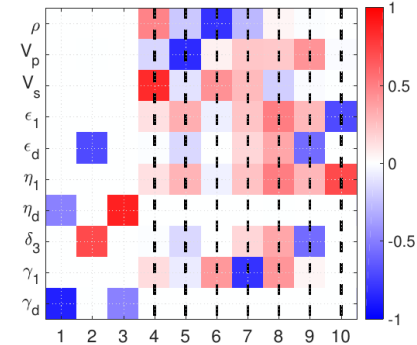

(c)

Figure 20. Same as Fig. 10, but for $S V-S H$ scattering. All VTI parameters don't scatter in this mode. Apart from this, $\delta_{3}$ and $\epsilon_{d}$ are present only in one singular vector corresponding to the second singular value and therefore coupled. 